\title{
Determining Mechanical Properties at the Micron Scale Using Microfabricated Freestanding Structures
}

\author{
A Dissertation Presented to the \\ Faculty of the School of Engineering and Applied Science \\ University of Virginia \\ In Partial Fulfillment \\ of the Requirements for the Degree of \\ Doctor of Philosophy \\ Mechanical and Aerospace Engineering
}

John Thomas Gaskins

August, 2013 
APPROVAL SHEET

The dissertation is submitted in partial fulfillment of the requirements for the degree of

Doctor of Philosophy in Mechanical and Aerospace Engineering

John T. Gaskins

This dissertation has been read and approved by the Examining Committee:

Matthew R. Begley, Advisor

N. Scott Barker, Advisor

Patrick Hopkins, Chairman

Hilary Bart-Smith

Michael L. Reed

Carl Knospe

Accepted for the School of Engineering and Applied Science:

James H. Aylor, Dean

School of Engineering and Applied Science

August, 2013 


\begin{abstract}
Thin films on the order of tens and hundreds of nanometers are being used with increasing frequency in a variety of applications including semiconductor electronics and microelectro-mechanical systems (MEMS). The mechanical properties of these films tend to deviate from those seen in bulk materials which has implications on device design, reliability and functionality. Naturally the size of these films leads to difficulty carrying out traditional mechanical testing, such as tensile or compression testing, to determine their properties. Indentation of free standing structures, specifically beams, has been identified as a means to test films in this size regime. This work focuses on development of indentation testing techniques, analytical solutions and fabrication methods to extract mechanical properties from free standing thin film beams.
\end{abstract}

Identification of the point of contact between the indenter probe and compliant beam is necessary in order to accurately determine loads and displacements carried by the beam and to enact time dependent loading schemes (e.g. constant strain-rate testing). Exploiting the under-damped resonant response of the indenter allows for identification of beams with stiffness up to two orders of magnitude less than the indenter springs. The indentation system is well characterized as a single DOF harmonic oscillator. Examining the coupled response of the indenter and beam, detection limits based on operating frequency, beam stiffness, beam damping and environmental noise in the system are identified.

An analysis of the full non-linear load-deflection response of point loaded elastic beams with tensile residual stress is presented. Relevant asymptotic limits corresponding to classical beam response are identified. Further, a simple closed-form expression is identified for non-linear responses that facilitates property extraction when asymptotic expressions 
are not valid. A critical contribution is the development of an explicit analytical relationship for load-deflection response which avoids the complication of using implicit solutions, which requires non-linear root-finding to determine the mechanical stretch in the beam. An approximate solution is shown to be accurate within $6 \%$ of the implicit solution.

Testing of npAu and AuAg beams and cantilevers is used to extract elastic modulus and residual stress. The proposed indentation testing techniques and approximate solution are used to extract mechanical properties from nickel beams created using a novel fabrication procedure utilizing $\mathrm{XeF}_{2}$ gas. The mechanical properties of films in their as-fabricated state and annealed at 200 and $300^{\circ} \mathrm{C}$ are explored. The effects of varying microstructure on modulus, residual strain, yield stress and fracture properties are discussed. Finally, areas of future study are addressed. 


\section{Acknowledgements}

I am grateful to Professor Matthew Begley for starting me on my graduate journey and helping with the completion of this project. I am equally endebted for the support provided by Professor Scott Barker who welcomed me to his group. I would like to thank Professors Patrick Hopkins, Hilary Bart-Smith, Michael Reed and Carl Knospe for input in the preparation of my dissertation and for the sacrifice of time to be on my committee.

Joe Beatrice, Alex Lobo and Harry Wade provided a wealth of knowledge and support in the cleanroom. Thanks to my colleagues who trained me on instruments and were available to hash out problems great and small including Dr. Erkin Şeker, Bob Benoit, Dr. Huihui Wang, Dr. Theodore Reck and the rest of the MIRFTech group. A huge thanks to the members of the administrative teams who have helped me through the years. Additionally it is necessary to acknowledge the inhabitants of the cleanroom who made multi-hour stints in a white room with no windows bearable.

The support of my friends here in Charlottesville as well as elsewhere has made the time pass more quickly. A huge thanks to my family for the love and support they have offered through the years. My final acknowledgement goes to my wonderful wife who has been a constant source of encouragement and has inspired me to push through to the end. 


\section{Contents}

Acknowledgements i

Nomenclature $\quad$ xi

$\begin{array}{lll}1 & \text { Introduction } & 1\end{array}$

1.1 Mechanical testing of beams . . . . . . . . . . . . . . . . . 3

1.2 Nanoindentation . . . . . . . . . . . . . . . . . . . . . 4

1.3 Review of work on freestanding beams . . . . . . . . . . . . . . . 6

1.4 Dissertation Structure $\ldots \ldots \ldots \ldots$. . . . . . . . . . . . . 8

$\begin{array}{lll}2 & \text { Indentation Theory } & 10\end{array}$

2.1 Quasi-static response of the indenter/specimen system . . . . . . . . . . . 11

2.2 Dynamic response of the indenter/specimen system . . . . . . . . . . . . . 14

2.3 Practical limitations $\ldots \ldots \ldots \ldots \ldots \ldots$

2.4 Constant strain rate testing . . . . . . . . . . . . . . 30

$\begin{array}{lll}3 & \text { Test Interpretation } & 35\end{array}$

3.1 Point loading fixed-fixed beams . . . . . . . . . . . . . 35

$3.1 .1 \quad$ Full solution and an effective approximation . . . . . . . . . . . 35

3.1 .2 Normalization . . . . . . . . . . . . . . . . 40

3.1 .3 Solutions . . . . . . . . . . . . . . . . . 42

3.2 Asymptotic limits . . . . . . . . . . . . . . . . . . . 44

3.3 Design of experiments . . . . . . . . . . . . . . . . . . . . . . . . . . . . . . 47

3.4 Geometric formulation . . . . . . . . . . . . . . . . . . . . . . . . . . 49

4 Material Properties of npAu and AuAg Films and Beams 51

4.1 Fixed-fixed beams . . . . . . . . . . . . . . . . 52

4.2 FIB-cut cantilevers . . . . . . . . . . . . . . . . 55

4.3 Film-on-substrate . . . . . . . . . . . . . . . . . 57

$5 \quad$ Fabrication and Film Characterization of Nickel Beams 60

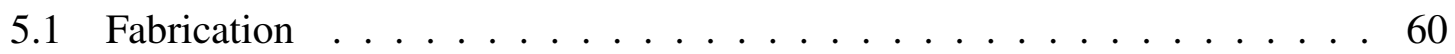

5.1 .1 Beam design . . . . . . . . . . . . . . 60

5.1 .2 Beam Fabrication . . . . . . . . . . . . . . . . . 63

5.1 .3 Alternative Fabrication Methods . . . . . . . . . . . . . . . . 64 
5.1 .4 Annealing protocols . . . . . . . . . . . . . 66

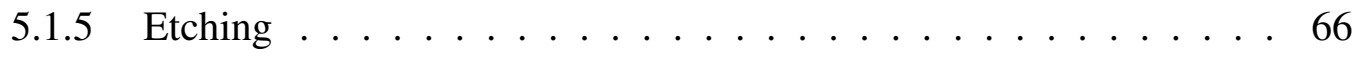

5.2 Length-scale characterization . . . . . . . . . . . . . . 70

5.2 .1 Geometric characterization . . . . . . . . . . . . . . 70

5.2 .2 Microstructural characterization . . . . . . . . . . . . 73

6 Elastic-plastic Properties of Nickel Beams $\quad 78$

6.1 Test parameters and protocols . . . . . . . . . . . . . . 79

6.2 Data post-processing . . . . . . . . . . . . . . . . . . . 82

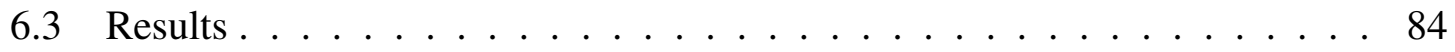

6.3 .1 Residual Strain . . . . . . . . . . . . . . . . . . . 84

6.3 .2 Modulus . . . . . . . . . . . . . . . . . . . 89

6.3 .3 Yield Stress . . . . . . . . . . . . . . . . . . . . 92

6.3 .4 Fracture . . . . . . . . . . . . . . . . . . . 94

6.4 Conclusion . . . . . . . . . . . . . . . . . . . . . . . 99

7 Conclusions and Future Work $\quad 100$

7.1 Indenter characterization . . . . . . . . . . . . . . . . 100

7.2 Test interpretation . . . . . . . . . . . . . . . . . 101

7.3 Experimental work $\ldots \ldots \ldots$. . . . . . . . . . . . . . . . . . . . .

7.4 Future work . . . . . . . . . . . . . . . . . . . . 102

8 Appendix 111

8.1 Photolithography Masks . . . . . . . . . . . . . . . . . 111

8.2 Fabrication procedure for freestanding beams . . . . . . . . . . . . . 112

8.3 Study on the tropical seed Mezzetia Parviflora . . . . . . . . . . . . . . 113

8.4 Beam Dimensions and Results . . . . . . . . . . . . . . . . . . . . . . . . 119 


\section{List of Figures}

1.1 Schematic of the indenter. The load coil is magnetically driven while the . 5

1.2 Beams used in a profilometry based technique (a) and beams from Es-

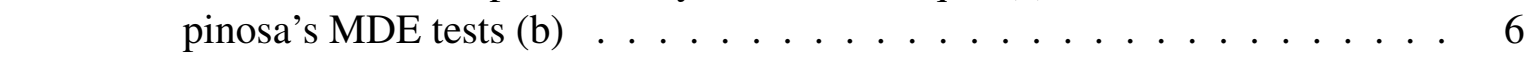

2.1 Schematic of indenter denoting relevant displacements and stiffness terms. . 11

2.2 Stiffness change as a function of missing the true contact under static loading. Softer materials (bottom curve) show large errors even when paired with a tip of large radius. . . . . . . . . . . . . . . . . . 13

2.3 Theoretical curves and experimental data for amplitude vs. frequency response of the DCM and XP heads. Solid points represent experimental data from indentation tests in free space. . . . . . . . . . . . . . . . . . 17

2.4 Amplitude response for the indenter in free space (purple) and in contact with differing values of $\kappa$ while $\eta$ is set to be zero. $\kappa=0$ represents the case where the indenter is oscillating in free space. . . . . . . . . . . . . 19

2.5 Amplitude and frequency response for various values of $\kappa$. The shift in amplitude response is more pronounced at low values of $\kappa$ than is the fre-

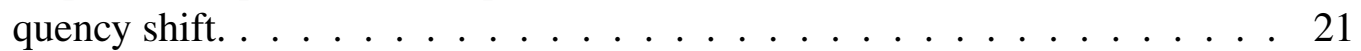

2.6 The effects of different stiffness ratios as a function of the damping ratio on the percent change in harmonic displacement upon establishing contact with $r=0.95$. The $\kappa=0$ curve implies a structure with zero stiffness can be detected if damping of the order of magnitude of that of the indentation system is present. It is also clear that structures with stiffness on the order of $1 \mathrm{~N} / \mathrm{m}$ provide a significant response, regardless of the level of damping

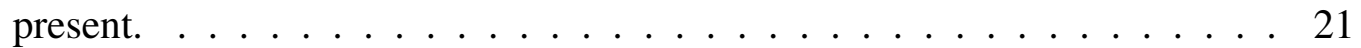

2.7 The effects of different damping ratios as a function of the stiffness ratio on the percent change in harmonic displacement upon establishing contact with $\mathrm{r}=0.95$. The red curve $(\eta=0)$ is indicative of the response of thin metallic beams where material and contact damping are negligible. . . . . . 22

2.8 Percent change for a few values of r. As expected the system becomes more sensitive to contact as the excitation frequency approaches the natural frequency of the system $(\mathrm{r}=1)$ except at low stiffnesses for operating frequencies just under the natural frequency. . . . . . . . . . . . 23

2.9 Data from nickel, $\mathrm{AuAg}$ and $\mathrm{npAu}$ beams and cantilevers showing good agreement between experimental data and theory using system parameters from the tests with eqn. 2.16 . . . . . . . . . . . . . . . . . 24 
2.10 Effects of varying system damping ratio on sensitivity to contact. The indentation system at UVa has system damping ratio of approximately $\zeta=$ 0.043 , similar to the green curve. . . . . . . . . . . . . 25

2.11 Harmonic displacement error as a function of system properties and error in harmonic load for $\zeta=0.043$. Operating closer to the natural frequency for the same error in harmonic load (i.e. noise/control error) increases the error in harmonic displacement. . . . . . . . . . . . . 26

2.12 Least squares fit to the error in harmonic displacement. The fit produces a harmonic load of $\sim 17 \mathrm{nN}$ which is the assumed environmental noise in the system. It should be noted that the harmonic displacement error in the tests near $r=0.85$ is not included in the analysis. The "noise" in the displacement signal at these frequencies has an oscillatory component of $\omega$ $=2 \mathrm{~Hz}$, implying the noise is coupled to an environmental vibration. . . . . 27

2.13 Experimental data on a $232 \mathrm{~nm}$ thick, $20 \mu \mathrm{m}$ wide by $500 \mu \mathrm{m}$ long nickel beam showing contact between sample and oscillatory component (B) and point where the sample is fully loaded (A). The transition regime between points $\mathrm{A}$ and $\mathrm{B}$ is $\sim 20 \mathrm{~nm}$ which is also the harmonic oscillation amplitude

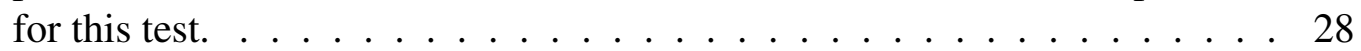

2.14 Experimental data on a $232 \mathrm{~nm}$ thick, $20 \mu \mathrm{m}$ wide by $500 \mu \mathrm{m}$ long nickel beam showing load-displacement response for different oscillation heights and approach speeds. Response is identical showing there is no adverse effect from large harmonic oscillations or varying approach speed. . . . . . 29

2.15 Representative diagram of stretch dominated MDE test. . . . . . . . . . . . 30

2.16 Representative velocity and strain rate profiles which ramp the velocity up to a transition point where the constant strain rate loading is then enacted. . 32

2.17 Difference in target strain rate versus that seen experimentally as a function of contact error and beam displacement. . . . . . . . . . . . . . 33

3.1 (a) SEM of representative MEMS beam used in point-load test. (b) Side view of coordinates and deformation variables used in the analysis. . . . . . 36

3.2 Exact and approximate load-deflection relationships for a broad range of

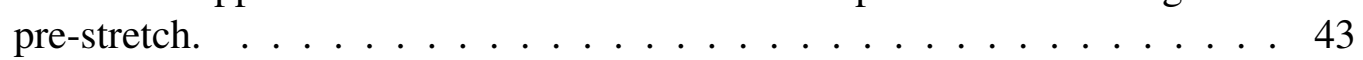

3.3 (a) The full range of $\mathrm{F}[\Lambda]$ and (b) error in the predicted load as a function of applied deflection for values of $\bar{\varepsilon}_{R}$ from $0-10^{6}$ when $c=2.12$. . . . . . 44

3.4 Illustration of combinations of (a) normalized critical loads, (b) normalized critical displacements and normalized pre-stretch for which asymptotic solutions are accurate: the shaded region represents the transition from linear regimes to the nonlinear regime where the analytical solution can be used to extract material properties. . . . . . . . . . . . . . . 46

3.5 Contours showing combinations of pre-strain and deflections where the contribution of bending strain to the total strain in the beam is 1,5 and $10 \%$. For applied displacements greater than $\sim 5$ times the film thickness bending strains are negligible regardless the level of pre-strain. . . . . . . . 47

3.6 Relevant loads, displacements and angles used to geometrically derive stress and strain . . . . . . . . . . . . . . . . 50 
4.1 Representative load-displacement curves on beams on $100 \mu \mathrm{m}$-long clamped npAu beams with various heat treatments . . . . . . . . . . . 52

4.2 npAu beam which has fractured during $400^{\circ} \mathrm{C}$ heat-treatment (a). Tensile beam failure of beam-clamp interface caused by skipping heat-treatment prior to dealloying discussed elsewhere[1] (b). Micro-crack formation during heat-treatment at $200^{\circ} \mathrm{C}$ (c). Micro-necking where a npAu beam has been broken with tweezers(d). Nanoindenter tip mark on electroplated $\mathrm{Au}$ clamp (e), and npAu beam surface (f).[2] . . . . . . . . . . . . . . 53

4.3 Residual stress as a function of pre-annealing for AuAg beams and npAu beams. The residual stress in the npAu beams is post dealloying, prior to additional annealing. . . . . . . . . . . . . . . 54

4.4 Stiffness of npAu FIB-cut cantilevers as a function of distance from the clamp which allow for independent determination of elastic modulus; curves are least-squares fit of the form $\mathrm{k}=\mathrm{c} / l^{3} \ldots \ldots \ldots 55$

4.5 Percent change of harmonic displacement as a function of beam stiffness and operating frequency for tests on $\mathrm{AuAg}$ and npAu beams. Lines running through data are theory from chapter 2. . . . . . . . . . . . . . . 57

4.6 Elastic modulus calculated from tests on $\mathrm{npAu}$ FIB-cut cantilevers and blanket FOS samples(a). Residual stress calculated from center span point loaded tests on fixed-fixed npAu beams and wafer curvature measurements on continuous films(b).[2] . . . . . . . . . . . . . 58

4.7 Residual stress as a function of pre-annealing for AuAg beams and as a function of post-annealing for npAu beams. Residual stress in the AuAg beams appears to reduce as a function of annealing temperature while the stress state in the npAu beams is variable depending on competing microstructural mechanisms. . . . . . . . . . . . . 58

5.1 Representative diagram of stretch dominated MDE test. . . . . . . . . . . . 61

5.2 Mask 3 used to define beam geometry. Positive resist profile defines evaporation, sputter and electroplating depositions. Negative resist profile can be used to etch beams from a continuous film. . . . . . . . . . . . . . 62

5.3 SEM image of filleted corner to reduce stress concentrations. . . . . . . . 63

5.4 Representation of lift-off stack for beam fabrication. . . . . . . . . . . . . . 64

5.5 Illustration of fabrication method for freestanding Nickel beams. (a) Side

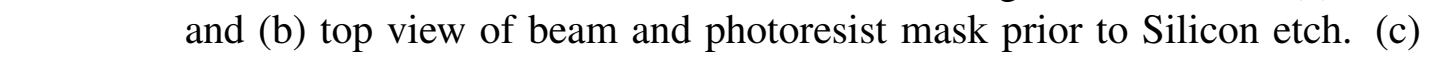
Side and (d) top view post-etch and lift-off resist removal. . . . . . . . . . . 65

$5.6 \quad$ Side (a) and top (b) views of the lithography and seed layer prior to electroplating. (c) Plated structure prior to PR and seed layer removal. . . . . . . 66

$5.7 \quad$ Original mask design (a) resulting in beams across an etched trench (b). . . 67

5.8 Films with microcracks from early beams where visible microcracks caused low beam yield from fabrication, low strength and reduced effective modulus when tested. . . . . . . . . . . . . . . 68

5.9 Photoresist mask prior to etching (a), representation of etched beam (b) and SEM of $300 \mu \mathrm{m}$ long by $20 \mu \mathrm{m}$ wide beam showing unetched silicon supports (c). . . . . . . . . . . . . . . . . 68 
5.10 Residual PR remaining on released beams. . . . . . . . . . . . . . . . . 69

5.11 a) Length measurement of $81 \mathrm{~nm}$ thick released beam. b) Width measurement of released beam post thermal treatment, before etching. . . . . . . 71

5.12 a) 2D contour map of beam where the difference between the darkest blue and red contours on the beam is less than one micron. Dashed line across beam center shows 2D cross-section seen below. b) 2D raw data along length of beam using the abrupt change in height due to the interface as the definition for the length of the beam. . . . . . . . . . . . . 72

5.13 a) Outlined grains allowing image to be converted to a binary image. b) Binary image which allows for automated grain size analysis. . . . . . . . . 74

$5.14232 \mathrm{~nm}$ thick film annealed at 300C for one hour. Larger grains appear to be forming, indicated by black arrows, while evidence of the smaller grains seen in the previous annealing conditions still exist. . . . . . . . . . . . 74

5.15 Nickel (111) peak for all thermal treatments (a) the full x-ray diffraction spectrum showing a highly textured film in the (1 111$)$ direction as well as the $\left(\begin{array}{lll}1 & 0 & 0\end{array}\right)$ silicon peak. . . . . . . . . . . . . . . . 76

6.1 Load and unload curves on 81nm thick unannealed beam where the maximum displacement corresponds to a strain of 0.002. Standard deviations are the average of 6 tests on the same beam showing identical behavior in the load and unload portions. . . . . . . . . . . . . . . . 79

6.2 Loading curves for (red) multiple test on the same beam averaged and (blue) three different beams averaged together. The similarity between curves suggests excellent repeatability from beam to beam and suggests the first loading to be indicative of the true response of the beam. . . . . . . 81

6.3 Stress strain behavior (a) and normalized load vs. displacement(b) . . . . . 83

6.4 Example of the procedure used to create stress strain curves and determine the flow stress. The black line is the linear extrapolation of modulus, the red line is the original stress strain curve which has been shifted to the right by an amount equal to the residual strain and the blue line is the modulus offset by $0.05 \%$ strain which is used to determine the flow stress. . . . . . . 83

6.5 Residual strain shows little variation between the as-fabricated and $200^{\circ} \mathrm{C}$ anneal but sees a large jump at $300^{\circ} \mathrm{C}$. The dashed lines represent the expected residual strain due to thermal mismatch strains where the deposition temperature is assumed to be room temperature. . . . . . . . . . . . 85

6.6 Timeline of thermal loads post deposition $\ldots \ldots$. . . . . . . . . 86

6.7 Evolution of strain as a function of the temperature seen during fabrication and annealing for the as-fabricated (a) and $200^{\circ} \mathrm{C}$ annealed beams (b). Annealing occurs before the mask is deposited for the etch procedure which influences the strain state in the beam for the $200^{\circ} \mathrm{C}$ annealed beams. . . . . 87

6.8 Evolution of strain as a function of the temperature seen during fabrication and annealing for the $300^{\circ} \mathrm{C}$ case where the beam is assumed to be unyielded (a) and yielded (b). Relaxation during the fabrication bakes is assumed to be neglible. . . . . . . . . . . . . . . . 88 
6.9 Volume fraction of grain boundary material as a function of grain size for three different grain boundary thicknesses (a). Theoretical and experimental modulus as a function of grain diameter. Modulus of the intergranular material and grain boundary material were taken as $205 \mathrm{GPa}$ and $100 \mathrm{GPa}$ and the grain boundary thickness appears to vary between 0.8 and $1.8 \mathrm{~nm}$ (b). 90

6.10 Modulus increase with annealing condition which is likely linked to grain size coarsening. The as-fabricated and $200^{\circ} \mathrm{C}$ annealed $42 \mathrm{~nm}$ thick films exhibit $\approx 20 \%$ lower modulus than the thicker films. . . . . . . . . . . . 91

6.11 Flow stress as a function of film thickness. Flow stress is defined as the intersection of the stress strain curve with a line of slope equal to the modulus, offset by $0.05 \%$ as seen in figure 6.4 . . . . . . . . . . . . . . . . 92

6.12 Flow stress as a function of square root of inverse grain size. There appears to be a transition from HP to RVHP behavior around a grain size of $18 \mathrm{~nm}$. The lines are added as visual aids. . . . . . . . . . . . . . . . . 93

6.13 Averaged stress strain curves for all tests. Blue lines represent as-fabricated, orange the $200^{\circ} \mathrm{C}$ and red the $300^{\circ} \mathrm{C}$ case. The final data point in each curve is the average fracture stress and fracture strain from the individual tests. . . 95

6.14 SEM of $81 \mathrm{~nm}$ as-fabricated(a) and $300^{\circ} \mathrm{C}(\mathrm{b})$ annealed fracture surface from a tilt angle of 45 degrees. Close examination of the as-fabricated fracture shows evidence of plasticity. The $300^{\circ} \mathrm{C}$ film appears to undergo brittle fracture which is expected from the stress strain curve. . . . . . . . . . 96

6.15 As-fabricated fracture surfaces $\ldots \ldots \ldots$. . . . . . . . . . . . . . . . . . . . . . .

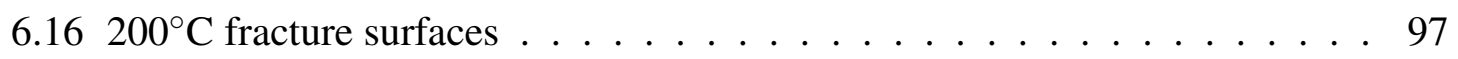

$6.17300^{\circ} \mathrm{C}$ fracture surfaces $\ldots \ldots \ldots$. . . . . . . . . . . . 98

6.18 Fracture stress as a function of fracture strain for all cases. Following the color convention from previous graphs, blue is as-fabricated, orange $200^{\circ} \mathrm{C}$ anneal and red $300^{\circ} \mathrm{C}$ anneal. Fracture strain is defined as the fracture strain from post-processed (shifted) stress strain curves. . . . . . . . . . . . . . 98

8.1 Beam masks for fabrication. Mask three was used for all beams in chapter 6. The etch masks (1a and 1b) contain varying difffering sizes of etch rectangles. Mask two was an experimental mask and was unused. . . . . . 111

8.2 Light microscopy of (a) short stubby cells in zone III, (b) parallel fibers of zone I (bottom of image) grading into randomly oriented zone II fibers (top), and (c) short stubby cells of zone III interspersed amongst random

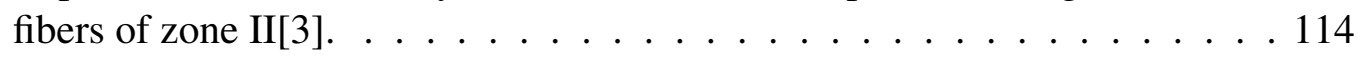

8.3 Modulus and hardness as a function of indenter displacement. Calibration values are valid for depths greater than $\sim 20 \mathrm{~nm} . \quad \ldots . . . . . . .115$

8.4 Modulus and hardness in the randomly oriented fiber region (zone II). The large scatter at small depths reduces as larger volumes of the material are sampled and the response becomes indicative of the bulk material response. 115 
8.5 Architecture of M. parviflora. Areas where indentation is used to examine micro scale properties are zones I, II and III. Middle two nut diagrams show the woody plug and zone III band where the crack initiates during germination. The bottom two figure illustrate the germination process as the seed is ejecting the plug and hypocotyl[3]. . . . . . . . . . . . . . 117

8.6 Illustration of predation mechanisms by beetles (a), squirrels (b) and orangutans(c). All of these predatory mechanisms happen over different length scales varying from $\sim 1 \mu m$ to millimeters[3]. . . . . . . . . . . . . . . 118 


\section{List of Tables}

$5.1 \quad$ Nickel Grain and Crystallite Size $\ldots \ldots \ldots$. . . . . . . . . . 77

$8.1 \quad$ Mechanical properties of M. parviflora for different locations. Mean (s.d.) .116

$8.2 \quad 42 \mathrm{~nm}$ beam dimensions $\ldots \ldots \ldots \ldots$

$8.381 \mathrm{~nm}$ beam dimensions $\ldots \ldots \ldots \ldots$

$8.4 \quad 232 \mathrm{~nm}$ beam dimensions $\ldots \ldots \ldots \ldots$

$8.5 \quad 377 \mathrm{~nm}$ beam dimensions $\ldots \ldots \ldots$. . . . . . . . . . . . . 121

8.6 As-fabricated Elastic Modulus and Residual Strain . . . . . . . . . . . 121

8.7 200C Annealed Elastic Modulus and Residual Strain . . . . . . . . . 122

8.8 300C Annealed Elastic Modulus and Residual Strain . . . . . . . . . 123

8.9 Results from membrane formulation . . . . . . . . . . . . . . . . 124 


\section{Nomenclature}

$\bar{\varepsilon}_{R} \quad$ Normalized residual strain in the beam

$\bar{\varepsilon}_{\text {bending }}$ Normalized bending strain in the beam

$\bar{\varepsilon}_{\text {max }} \quad$ Maximum normalized strain in the beam

$\bar{P} \quad$ Load normalized by $P_{0}$

$\bar{u} \quad$ Axial displacement normalized by half the beam length

$\bar{w} \quad$ Transverse displacement normalized to beam thickness

$\bar{x} \quad$ Distance along beam normalized to beam length

$\beta \quad$ Full width at half maximum intensity

$\Delta \alpha \quad$ Difference in coefficients of thermal expansion of the substrate and film

$\Delta T \quad$ Difference between the annealing (or maximum) temperature and the deposition temperature

$\Delta x \quad$ Harmonic oscillation height in free space

$\Delta x_{c} \quad$ Harmonic oscillation height after contact with the beam/sample

$\Delta \quad$ Deflection of the load point at the center of the beam normalized by beam thickness

$\Delta_{e} \quad$ Error in displacement associated with indenter transitioning from tapping surface of the sample to being in full contact

$\delta_{f} \quad$ Displacement of the load-frame

$\Delta_{g} \quad$ Grain boundary thickness

$\delta_{p} \quad$ Indenter penetration of the indenter into the specimen

$\delta_{s} \quad$ Deflection of the compliant structure, referenced to its position prior to contact

$\dot{\delta} \quad$ Displacement rate of the beam

$\dot{\varepsilon} \quad$ Strain rate imposed on the beam 
$\dot{\varepsilon}_{a} \quad$ Strain rate experienced by the beam taking into account error in contact

$\dot{\varepsilon}_{t} \quad$ Target strain rate used to calculate displacement rate during testing

$\varepsilon \quad$ Total axial strain in the beam

$\varepsilon_{f} \quad$ Final strain in the beam after thermal cycling from annealing and mask deposition

$\varepsilon_{i} \quad$ Intrinsic residual strain

$\varepsilon_{R} \quad$ Mechanical strain in the beam at zero elongation caused by tensile residual stress during fabrication

$\varepsilon_{t} \quad$ Thermal residual strain from mismatch of CTE between film and substrate

$\eta \quad$ Ratio of sample damping to indenter damping

$\Gamma \quad$ Ratio of the harmonic oscillation (missed contact) to beam displacement

$\kappa \quad$ Ratio of sample stiffness to indenter stiffness

$\Lambda \quad$ Normalized mechanical strain in the beam

$\lambda \quad$ Square root of spatially uniform mechanical strain in the deformed state

$\lambda_{K \alpha 1} \quad \mathrm{X}$-ray wavelength

$\omega \quad$ Operating frequency of the indentation system

$\omega_{n} \quad$ Indenter natural frequency

$\phi \quad$ Phase shift of harmonic oscillation

$\sigma \quad$ Stress in the beam

$\sigma_{R} \quad$ Residual stress in the beam from fabrication

$\sigma_{Y B} \quad$ Bulk yield stress

$\sigma_{Y S} \quad$ Size dependent yield stress

$\theta \quad$ Angle between the undeformed and deformed states of the beam

$\theta_{o} \quad$ Bragg angle

$\zeta \quad$ Damping ratio of the indentation system

A Area of beam cross section

A Area under the curve of the gaussian fit

c Numerical approximation of $F(\Lambda)$ used to determine an approximate solution 
$D_{c} \quad$ Damping associated with the tip/surface interface (contact)

$D_{i} \quad$ Damping of the indentation system

$D_{\text {grain }}$ Diameter of the grain size via Scherrer analysis

E Young's modulus

$E_{e} \quad$ Effective modulus of the specimen and indenter tip modulus

$E_{c} \quad$ Composite modulus of the grain boundary and intergranular material

$E_{g b} \quad$ Modulus of the grain boundary

$E_{i g} \quad$ Modulus of the intergranular material

$F(\Lambda)$ Prefactor to $\Delta^{2}$ in solution of mechanical strain in the beam in the deformed state.

$F_{0} \quad$ Harmonic driving force

$h \quad$ Beam thickness

$H(i \omega)$ Complex frequency response of indentation system

I Area moment of inertia

$K \quad$ Dimensionless shape factor taken as 0.9

$k \quad$ Strengthening coefficient

$k_{f} \quad$ Stiffness of the load frame

$k_{i} \quad$ Stiffness of the indenter column

$k_{s} \quad$ Stiffness of the sample

$L \quad$ Beam length

$l \quad$ Half-length of the deformed beam section

$m \quad$ Mass of the indentation system

$P \quad$ Point load applied at beam center

$P_{0} \quad$ Load at which the classical beam deflection result equals one beam thickness

$P_{m} \quad$ Load in the cross-section of the beam

$P_{S} \quad$ Load transferred to the sample

$P_{\text {raw }} \quad$ Raw load

$R \quad$ Radius of the indenter tip 
$r \quad$ Frequency ratio of the indentation system

$T$ Annealing temperature or maximum temperature during fabrication

$T_{d} \quad$ Temperature at deposition

$u \quad$ Axial displacement in the beam

$V_{g b} \quad$ Volume of grain boundary material

w Transverse beam displacement

$x \quad$ Distance along beam length

$x_{c} \quad$ Position of the indenter when contact is first established

$x_{\text {raw }} \quad$ Raw position of the indenter

$y \quad$ Distance from the centerline of the beam

$y_{o} \quad$ Background noise in XRD signal 


\section{Chapter 1}

\section{Introduction}

When examined at the microscopic scale many things we use everyday are made of or have components that use thin films. Thin films are used in a wide range of applications including semiconductor microelectronics, microelectro-mechanical systems (MEMS) and chemical sensors. Nickel thin films in particular have been used in nickel metal hydride battery systems [4], an in-situ repair process of nuclear steam generator tubing[5], microfiltration systems [6] and in the area of soft magnets [4, 7]. The mechanical properties of these films with regard to applications are of interest to industry in order to better design devices, increasing reliability and functionality.

Many properties of thin films, including mechanical, optical, magnetic, and electronic, deviate from those of bulk when their dimensions become small[8, 9]. Often these deviations can be directly linked to the dimensions or microstructural sizes in the film approaching length scales where defects, grain boundary deformation or small scale dislocation mechanisms become relevant[10]. Mechanical properties have been known to deviate from bulk properties at relatively "large" length scales, around a micron[11]. These property deviations include but are not limited to increases and decreases in elastic modulus, yield stress, ductility and strength. 
The increase of interest in properties on these length scales has driven a need to accurately characterize and link both microstructural and geometrical scaling to thin film properties. Naturally the size of these films leads to difficulty carrying out "traditional" mechanical testing such as tensile and compression testing. Experimental difficulties at this size of films include device fabrication, handling of specimens, complicated alignment schemes and monitoring and controlling ultra low loads and displacements[12,-14]. New testing techniques are continuously being developed in an effort to shed light on the various mechanisms that control the differing properties.

Among the most commonly used methods of characterization is nanoindentation because of its ability to span a wide range of loads and displacements[15]. Traditionally nanoindentation has been used to probe the properties of films on substrates (FOS) ${ }_{1}^{1}$ with high stiffness such that the determination of contact between the instrument and film being probed is a trivial matter. Within the past decade the uses of nanoindentation have expanded to include testing of ultra-compliant films such as polymers, structures and thin films with thickness less than a micron where contact identification becomes significantly more difficult[12, 16, 17].

Testing of MEMS beams has presented itself as an excellent technique to extract the mechanical properties of ultra thin metallic films[1, 2, 12, 18]. By creating beams with long spans, they can be deflected such that the resultant test is analogous to a uni-axial stress-strain test. A wide range of stress and strain can be probed by modulating combinations of beam length and thickness. The details of these tests including experimental protocols needed to identify contact between the indentation system and sample, theoretical derivations for point-loading of residually stressed beams and extraction of mechanical properties will be discussed. The derivation and adaptation of the point-load solution to this problem removes the common experimental issues of alignment and allows for prop-

\footnotetext{
${ }^{1}$ There is a comprehensive FOS study in Appendix 8.3 on the tropical seed Mezzetia Parviflora showing an example of evolutionary mechanical "optimization".
} 
erty extraction from nearly any suspended beam.

\subsection{Mechanical testing of beams}

An effective way to measure material properties for thin films is to measure the loaddeflection relationship of a freestanding structure, such as a thin beam or wire (e.g. membranes [12, 16, 19, 20], microbeams[21, 22] and wires[23, 24]). Such methods are increasingly popular because a wide range of loads and displacements can be accessed using existing off-the-shelf systems, such as atomic force microscopes (AFM) [23-29], nanoindentation systems, profilometers and instrumented micro-indenters [1, 2, 12, 16, 19-[22]. Further, the preparation of specimens amenable to one-dimensional modeling (i.e. beams and wires) is increasingly straightforward with emerging micro- and nanofabrication techniques [30, 31] . Arguably the simplest and most accurate method to determine properties using this approach is to choose a combination of loads, displacements and specimen geometry such that closed-form analytical expressions for load-deflection curves are accurate.

There are several classical limits regarding the mechanical response of beams with explicit analytical solutions, each corresponding to different combinations of applied loads, modulus and residual stress in the beam[32]. The challenge is to identify the combination of properties that corresponds to each limit, such that an appropriate solution is used to extract properties. For example, linear load-deflection relationships can result from either bending dominated behavior (small values of residual stress and small displacements), or from stretch dominated behavior (i.e. large values of residual stress). Non-linear load-deflection relationships can be obtained for scenarios where deflections are large, but the threshold of 'large' displacements naturally depends on the level of pre-stretch, and so forth. Each of these limits is of interest, as each enables straightforward extraction of various material properties; for example, linear response is useful to extract elastic modulus and residual 
stress, while large deflections (in which bending is negligible) can facilitate the measurement of non-linear material response by ensuring uniform stretching in the beam.

Further, the ability to identify various regimes of response is critical to the accurate design of actuators and sensors, such that the appropriate input/output relationships are used. Simply put, given a range of expected properties or desired performance, what dimensions should be chosen in conjunction with a specific instrumentation system (be it for measurement, sensing or actuation) to ensure the accuracy of analytical solutions? For example, one might like to know the the combination of deflections and beam sizes that can be chosen to ensure that bending strains are negligible, such that the test can be modeled assuming pure stretching of the beam. These considerations are increasingly important as researchers attempt to decrease the dimensions of test structures down to the nanometer scale[23, 24].

\subsection{Nanoindentation}

The two most commonly used commercial nanoindenters are made by Agilent Technologies(Chandler, AZ) and Hysitron, Inc. (Minneapolis, MN). All work in this dissertation was performed with a NanoIndenter ${ }^{\circledR}$ DCM made by Agilent Technologies. Raw force and displacement for most available commercial systems are coupled to leaf springs[33] and as such all theory, measurement methods and analysis techniques presented are generally applicable to any indentation system.

Figure 1.1 shows a schematic of a typical nanoindenter. The load is applied by a magnetic coil while the displacement is measured via changes in capacitance. In most cases indentation systems are load controlled, making use of signal feedback to achieve the desired load/displacement. It should be noted the control systems and feedback loops are often inaccessible to users. The springs carry the load and are linear over the travel range 


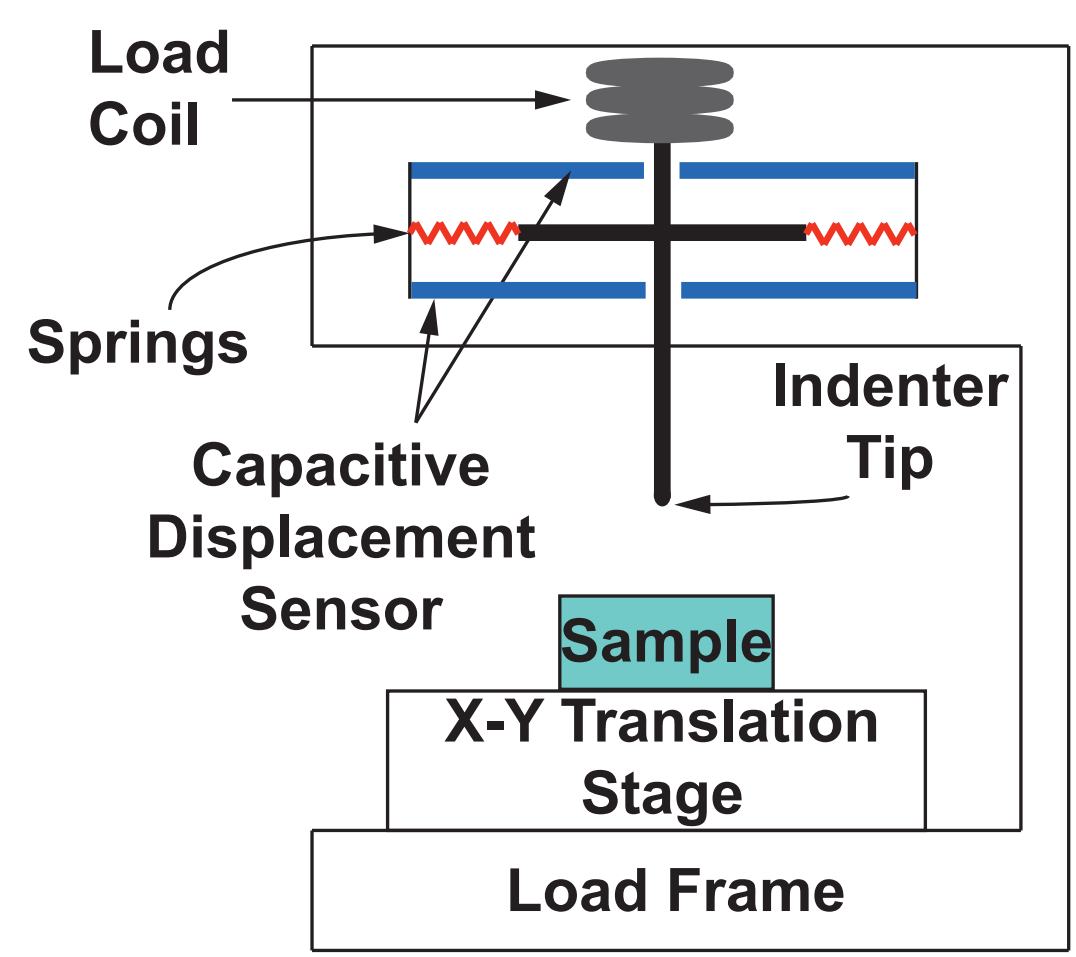

Figure 1.1: Schematic of the indenter. The load coil is magnetically driven while the of the indenter (i.e. the raw load-displacement signal in free space is characteristic of the spring stiffness).

Traditionally nanoindentation has been used as a tool to extract the modulus and hardness from films on substrates with thickness on the order of $>200 \mathrm{~nm}[15$, 34]. Relationships between the contact area of the tip as a function of indentation depth and the contact stiffness allow for determination of elastic modulus and hardness as a function of indentation depth. Over the past decade use of the nanoindenter has expanded to include testing of structures to extract the mechanical properties of freestanding films. The ability to accurately determine loads and displacements in the nanometer and nano/micronewton regime makes it a useful tool to investigate the properties of micro and nano-scale structures. 

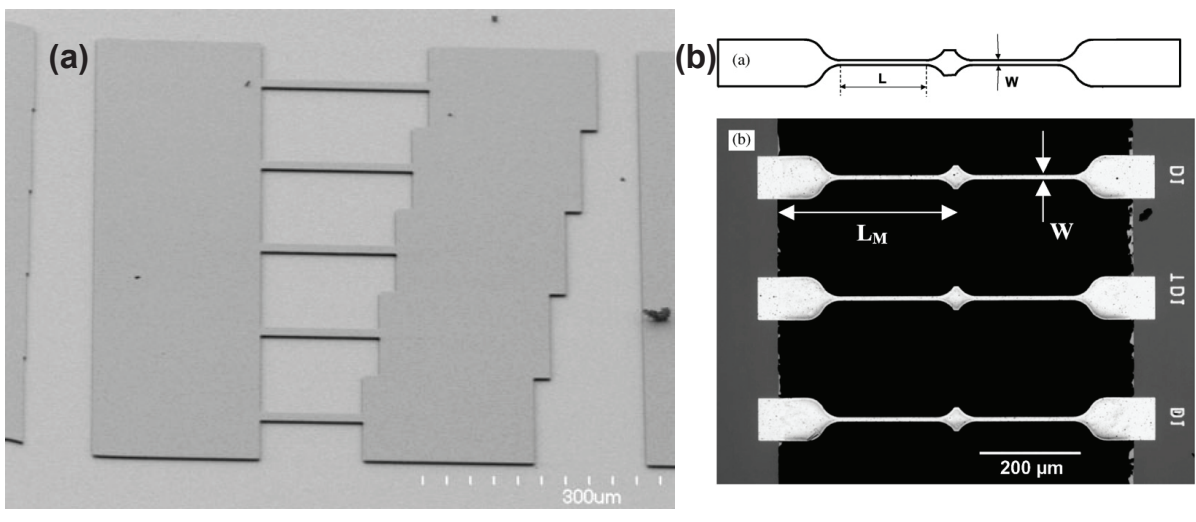

Figure 1.2: Beams used in a profilometry based technique (a) and beams from Espinosa's MDE tests (b)

\subsection{Review of work on freestanding beams}

One of the first works utilizing indentation of fixed-fixed beams was performed by Zhang et. al.[16]. Freestanding beams of silicon nitride were indented with a wedge shaped indenter across the thickness of the film. An analytical solution in the bending regime along with finite element analysis (FEA) was used to extract modulus and residual stress. No mention was made of the possible effects of indenter misalignment across the face of the film which would have negative consequences if not properly aligned. The maximum deflection normalized by the film thickness was in the range of ten, an order of magnitude smaller than the work presented here.

A profilometry based technique was proposed by Denhoff[21]. Young's modulus and residual stress were extracted by running a surface profilometer across the length of a beam similar to those seen in figure 1.2 a. Paired with an analytical beam theory solution, again in the bending regime, as a function of distance along the beam, Young's modulus and residual stress were determined. There was ambiguity as to the effective beam length due to over etching and the alignment process along the length of the beam was relatively poor, within $5 \mu \mathrm{m}$.

Espinosa et. al. performed membrane deflection experiments (MDE) on Au freestand- 
ing beams[12]. Utilizing specialized beams, seen in figure $1.2 \mathrm{~b}$, a line load tip on a nanoindenter was applied at the middle of the span. The deflection of the beam was recorded by a Mirau microscope interferometer positioned under the membrane. This required complicated micromachining steps to open a window under the suspended beams. Additionally the beams created in this procedure have specialized geometry to assist with the test and thus are only useful in this testing configuration. Stress and strain were computed independently by pure geometric relationships. As with the work by Zhang, there was an alignment necessary between the membrane and the indentation tip prior to testing. This work has the distinction of being able to produce complete stress-strain curves for a material but requires a difficult experimental setup.

Herbert et. al. have developed a procedure utilizing the continuous stiffness measurement of an MTS indenter to measure the elastic properties of thin films[20]. Measuring the stiffness vs. displacement response of a beam, results are analyzed using a membrane stretching model which is derived under the assumption of small strains (i.e. elastic deformation). This method again requires an alignment procedure between the line load tip used to impart the load to the beam surface. A number of experimental pitfalls such as thermal drift were addressed and as a result have been addressed in the following work.

It is important to note all of the work above provides useful insight into the mechanical properties of thin films. However, they each seem to hold a pitfall such as solutions restricted to a specific testing regime (bending, membrane stretching, etc.), complicated fabrication processes or involved alignment processes. Additionally, none of the existing work tests beams at constant strain rate[1, 2, 12, 16, 19,-22]. While this is less of a concern for the cases where elastic properties are extracted, permanent deformation based properties such as yield stress and fracture properties are often linked to the rate of testing [35-38]. An experiment including fabrication, test method and data interpretation which eliminates all of the challenges above and extends the MDE to constrant strain-rate testing is presented 
in this dissertation.

\subsection{Dissertation Structure}

Chapter 2 examines the nanoindentation system as a single-degree of freedom harmonic oscillator. Utilization of the dynamic characteristics of this system and their relevance to testing freestanding structures as well as compliant thin films is discussed. In addition, testing of beams using constant strain rate and some of the practical limitations of nanoindentation testing are examined. Chapter 3 takes an in depth look at the mechanics governing point-loaded deflection of microscale beams under residual tension. A comprehensive solution and effective approximation are presented that allows experimental data to be fit over the full range of an experimental test, with $\pm 6 \%$ error, irrespective of the well established regimes (prestressed plate, prestressed membrane or pure membrane stretching) through which the experimental data runs. The design of tests to extract properties using these solutions is addressed. Stress and strain derived from a geometric viewpoint with the assumption of membrane stretching is presented. These analyses show that a point load is sufficient to extract mechanical properties and recreate traditional stress-strain curves, drastically reducing difficulty of test set-up and ambiguity regarding sample alignment with the indentation probe. In chapter 4 experiments on nano-porous gold(npAu) and gold-silver alloy (AuAg) beams are used to show the effectiveness of the surface find method developed in chapter 2 and to examine the difference in mechanical properties between FOS, fixedfixed beams and cantilevers as a function of varying annealing temperature. In chapter 5 a fabrication procedure used to create nickel beams and the characterization techniques used to examine geometric and microstructural properties are presented. Chapter 6 presents results on nickel beams with thickness of 42, 81, 232 and $377 \mathrm{~nm}$. Three annealing conditions, as-fabricated, $200^{\circ} \mathrm{C}$ for one hour and $300^{\circ} \mathrm{C}$ for one hour, are presented to examine the change in mechanical properties as a function of temperature. Mechanical properties 
such as elastic modulus, residual strain, yield stress, fracture stress and fracture strain are shown to vary as a function of grain size and film thickness. Finally, chapter 7 summarizes the contributions, both experimental and theoretical, related to the use of microfabricated beams in extracting mechanical properties. Future work and areas of further exploration are addressed. 


\section{Chapter 2}

\section{Indentation Theory}

A schematic of the indentation system comprising the indenter head mounted to a frame with flexible springs, a load-frame that holds the head and specimen mount, and a beamlike specimen is shown in figure 2.1. The springs and dash-pots are used to represent the stiffness and damping introduced by the indenter head, load frame, and sample. In the following, the bottom of the system (i.e. the load frame) is assumed to be fixed: the indenter head mounts to a fixed reference system as well, but the mass of the head is displaced by an applied force.

The central challenge in the present approach is to determine (a) the load carried by the sample - that is, the portion of the applied (raw) load that is not carried by the indenter mounting springs, and (b) the deflection of the sample relative to its undeformed state, preferably excluding the localized effects of the indenter penetrating the sample. That is, one wishes to extract the load on the sample, $P_{S}$, and the subsequent deflection of the compliant structure, $\delta_{s}$. Here, we use the term "penetration" to refer to the local deformation of the specimen near the indenter: this is specifically defined as the difference in the total displacement of the top surface of the specimen and the total displacement of the bottom surface of the specimen. That is, if the indenter does not "dig in" to the surface of the specimen, but rather simply displaces the structure of the specimen, the penetration is zero. 


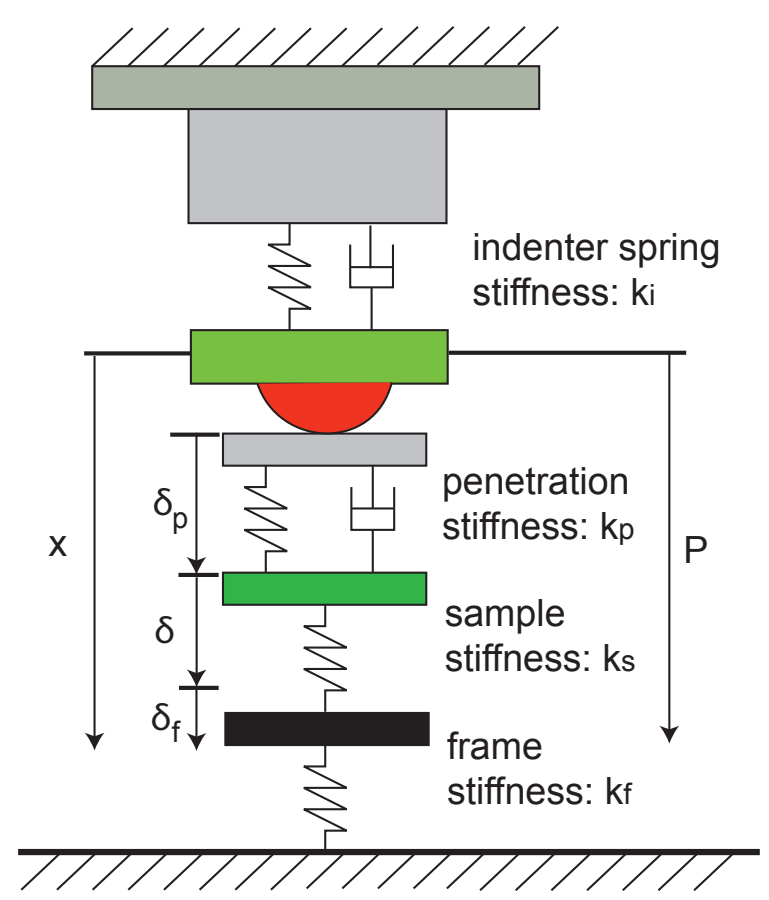

Figure 2.1: Schematic of indenter denoting relevant displacements and stiffness terms.

\subsection{Quasi-static response of the indenter/specimen system}

For simplicity, first consider the quasi-static response of the system, which implies the damping (rate-dependent) response of the system is neglected. The raw load applied to the indenter mass is denoted as $P_{\text {raw }}$, while the load transferred to the sample is denoted as $P_{s}$. Equilibrium in the loading direction dictates that the load on the sample is given by:

$$
P_{s}=P_{\text {raw }}-k_{i} \cdot x_{\text {raw }}
$$

where $k_{i}$ is the stiffness of the indenter springs and $x_{\text {raw }}$ is the raw position of the indenter measured relative to the zero-force position of the head. This result implies that in order to determine the load on the specimen with great precision, the raw position of the head and stiffness of the indenter springs must be known with great precision.

In order to determine the deflection of the compliant structure, one must identify the raw position of the indenter when contact is first established, denoted here as $x_{c}$. When the 
indenter is in contact with the specimen, the indenter's displacement relative to the contact point is equal the total displacement of the objects underneath. This can be expressed as:

$$
x_{\text {raw }}-x_{c}=\delta_{f}+\delta_{p}+\delta_{s}
$$

where $\delta_{f}$ is the displacement of the load-frame, $\delta_{p}$ is the penetration displacement of the indenter into the specimen, and $\delta_{s}$ is the deflection of the compliant structure of the specimen referenced to its position prior to contact. Equilibrium dictates that the load is uniform through the stack. Here, it is assumed that the load-frame exhibits a constant stiffness, denoted as $k_{f}$, while the indenter penetration can be estimated by Hertzian contact

$$
x_{\text {raw }}-x_{c}=\frac{P_{s}}{k_{f}}+\left(\frac{9 P_{s}^{2}}{16 R E_{e}^{2}}\right)^{1 / 3}+\delta_{s}
$$

where $R$ is the radius of the indenter tip, and $E_{e}$ is an effective modulus that depends on the modulus of the specimen and the indenter tip modulus. Assuming the contact point, $x_{c}$, is known, eqns. 2.1 and 2.3 provide the basis to use the raw $\left(P_{\text {raw }}, x_{\text {raw }}\right)$ signal to estimate the relationship between the load on the sample, $P_{s}$ and the specimen deflection, $\delta_{s}$. It should be emphasized that it is the sample loads and displacements, $\left(P_{S}, \delta_{S}\right)$ that are used with mechanical models of the specimen to determine its mechanical properties.

Eqn.2.3 clearly illustrates that the raw position associated with contact $\left(x_{c}\right)$ must be determined in order to calculate the $\left(P_{S}, \delta_{S}\right)$ values that are used to assess the mechanical response of the sample. Since an independent accurate determination of the raw position of the surface of the sample relative to the indenter's zero force position is impractical, the contact point $x_{c}$ must be inferred from the raw load-displacement signal, i.e. the $\left(P_{\text {raw }}, x_{\text {raw }}\right)$ dataset collected during the experiment. For specimens whose stiffness is small compared to the indenter springs, this represents a considerable challenge. To understand this, consider the simple scenario where the indenter penetration is zero: prior to contact, the slope of the raw load-displacement curve is $k_{i} \sim 85 \mathrm{~N} / \mathrm{m}$. After contact, the slope changes to 


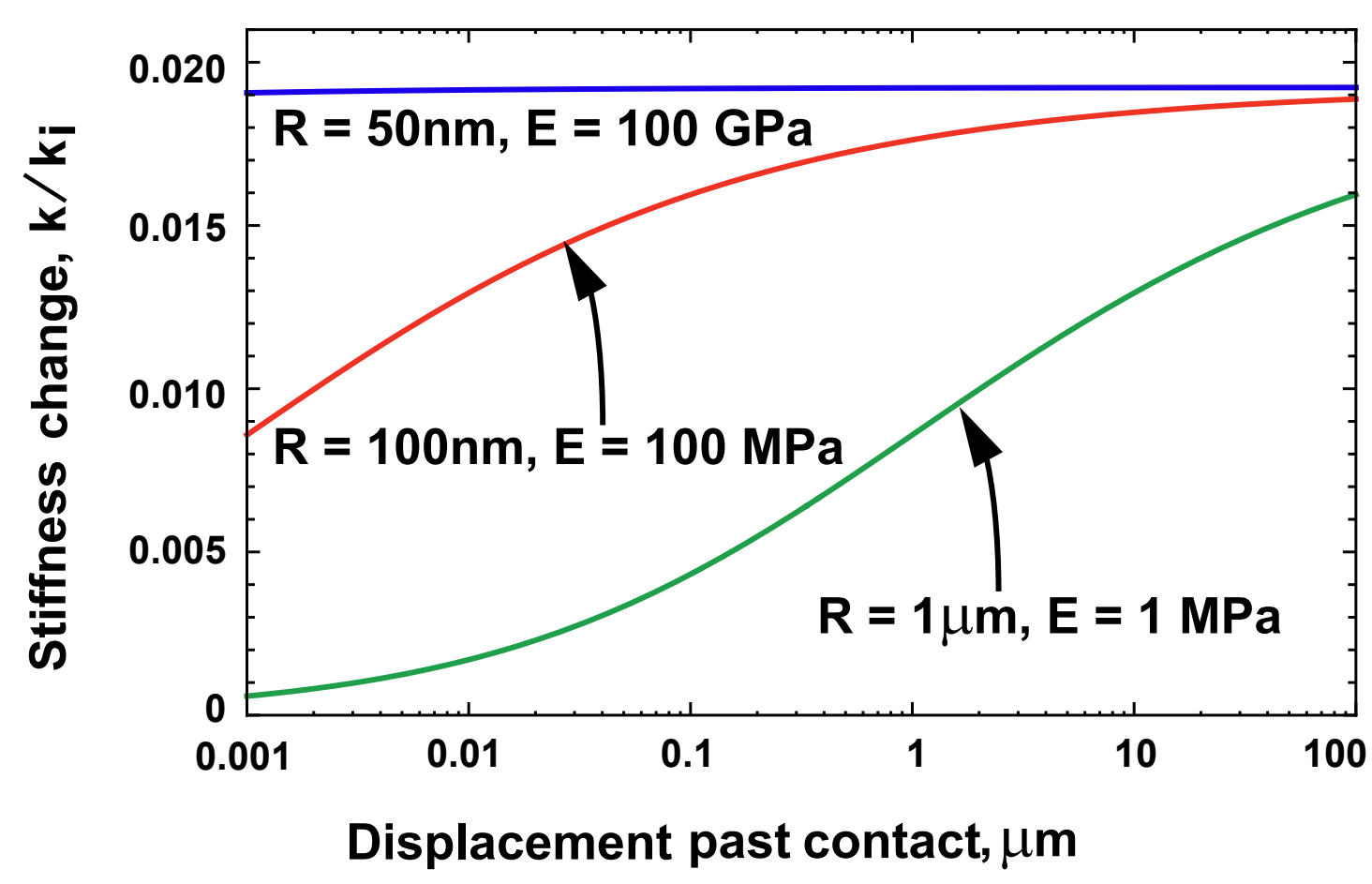

Figure 2.2: Stiffness change as a function of missing the true contact under static loading. Softer materials (bottom curve) show large errors even when paired with a tip of large radius.

$k_{i}+k_{s}$, where $k_{s}$ is the stiffness of the sample. For compliant specimens, such as microfabricated beams, if the stiffness of the specimen (e.g. a microfabricated beam) is $\sim 1.5 \mathrm{~N} / \mathrm{m}$, the change in slope due to contact is slightly under $2 \%$. Such small changes are difficult to distinguish from experimental noise, particularly when quasi-static measurements are utilized.

The difficulties associated with using changes in raw load-displacement data (i.e. stiffness) are compounded for sharp indenters or soft materials, which are prone to indenter penetration. For such scenarios, the stiffness of the indenter tip/surface interaction may be small compared to that of the structure. The implication is that it takes a finite amount of raw displacement to raise the tip/surface stiffness to the point that it is effectively rigid, such that one observes the stiffness of the underlying structure. This is illustrated in Figure 
2.2, which plots the observed stiffness change as a function of displacement past the true contact point. This is calculated by inverting eqns. 22.1,2.3 and differentiating to calculate $d P / d x$. Several cases are shown with different tip radii and modulus. The bottom curve (for a micron radius tip on an elastomer) illustrates that even if small changes in stiffness can be detected (say $\sim 1 \%$ ), the position of the head associated with contact will be off by as much as a micron. The top curve is for a sharp indenter $(\sim 50 \mathrm{~nm})$ on metals: this illustrates that the stiffness of the tip/surface interaction rises quickly with the load on the sample, such that the system stiffness is dominated by the spring/sample interaction.

\subsection{Dynamic response of the indenter/specimen system}

The difficulties associated with detecting contact via changes in the raw load-displacement data can be addressed by exploiting dynamic excitation of the indenter head. This approach has two key advantages: (i) the use of harmonic excitation (and the associated response) significantly reduces signal-to-noise ratios as compared to quasi-static approaches, because the data processing relies on averaged response over repeated cycles as opposed to differentiation, and (ii) the effective stiffness of the indenter springs can be reduced by exploiting the under-damped resonant response of the head/spring assembly. The latter has the effect of bringing the probe stiffness and sample stiffness into closer alignment (magnitude-wise), which leads to more dramatic changes associated with contact.

The basic concept is to superpose an oscillatory (harmonic) force on the indenter with the macroscopic ramp loading that is needed to move the indenter head into contact. When the indenter contacts the specimen, the added change in stiffness reduces the oscillatory displacement of the indenter. In the experiments to be developed, it is the harmonic displacement of the indenter that is monitored, with changes in this quantity used to identify contact. The role of the ramp loading (and specifically, ramp loading rate) that moves the indenter into contact is ignored: since the focus is on the harmonic response, it is assumed 
that the ramp load is applied incrementally in a 'quasi-static' manner, i.e. at a slow enough rate as to not introduce transient terms into the dynamic response. It is assumed that the oscillatory component is small enough, and, the raw position of the indenter is sufficiently large, as to avoid loss of contact during the oscillation (i.e. "chatter"). Put another way, it is assumed that the raw position of the indenter is sufficient past contact such that unloading during harmonic oscillations does not lead to a loss of contact.

First, consider the dynamic response of the indenter head, subject to the harmonic forcing function $F(t)=F_{0} e^{i \omega t}$. For this case, the stiffness $k_{i}$, damping $D_{i}$, mass $\mathrm{m}$, operating frequency $\omega$ and harmonic force $F_{0}$ are those of the indentation system. Assuming the equation of motion for the single-degree of freedom system is:

$$
m \ddot{x}+D_{i} \dot{x}+k_{i} x=F_{0} e^{i \omega t}
$$

The steady-state amplitude of the indenter head's response is given by:

$$
X=\frac{F_{0}}{\left(k_{i}-m \omega^{2}\right)+i D_{i} \omega} .
$$

The phase shift between the excitation force and the system response is given by:

$$
\phi=\tan ^{-1}\left(\frac{D_{i} \omega}{k_{i}-m \omega^{2}}\right) .
$$

The complex frequency response of the system can be expressed as:

$$
\frac{k X}{F_{0}}=\frac{1}{1-r^{2}+i 2 \zeta r} \equiv H(i \omega)
$$


where $r$, the frequency ratio, and $\zeta$, the damping ratio, are

$$
\begin{aligned}
r & =\frac{\omega}{\omega_{n}} \\
\zeta & =\frac{D_{i}}{2 \sqrt{m k_{i}}} .
\end{aligned}
$$

The absolute value of $H(i \omega)$ gives the amplitude magnification factor, which describes the amplitude of the head as a function of frequency:

$$
|H(i \omega)|=\left|\frac{k X}{F_{0}}\right|=\frac{1}{\left[\left(1-r^{2}\right)^{2}+(2 \zeta r)^{2}\right]^{1 / 2}} .
$$

In terms of these normalized variables, the phase shift is given by:

$$
\phi=\tan ^{-1}\left(\frac{2 \zeta r}{1-r^{2}}\right)
$$

Figure 2.3 illustrates measurements dynamic response of the indenter head at various frequencies, with the above theory superposed: one can fit the theoretical response to the measured response to determine the mass, effective damping and natural frequency of the indenter head. The spring stiffness of the indenter can be determined by performing air indents and calculating the slope of the load displacement curve. For the system descried here the spring stiffness was $\sim 82 \frac{N}{m}$. Fitting experimental data, shown as circles in Figure 2.3. from tests performed at fixed oscillation amplitude and varying frequency yield system parameters: $\mathrm{m}=81 \mathrm{mg}, \mathrm{D}=7 \times 10^{-3} \frac{N-s}{m}$ and $\omega_{n} \sim 160 \mathrm{~Hz}$. Also shown is the response of the larger, stiffer XP head: note that this head is over-damped, where mass dominates over stiffness, and is hence unsuitable for the technique described here. It should be noted that damping is a function of the position of the indenter head. The effect of this variance in property as a function of indenter travel is explicitly addressed below. It is important to emphasize that system parameters quoted above are specific to the indentation unit at UVa. System properties may vary from system to system and may even be of different relative 


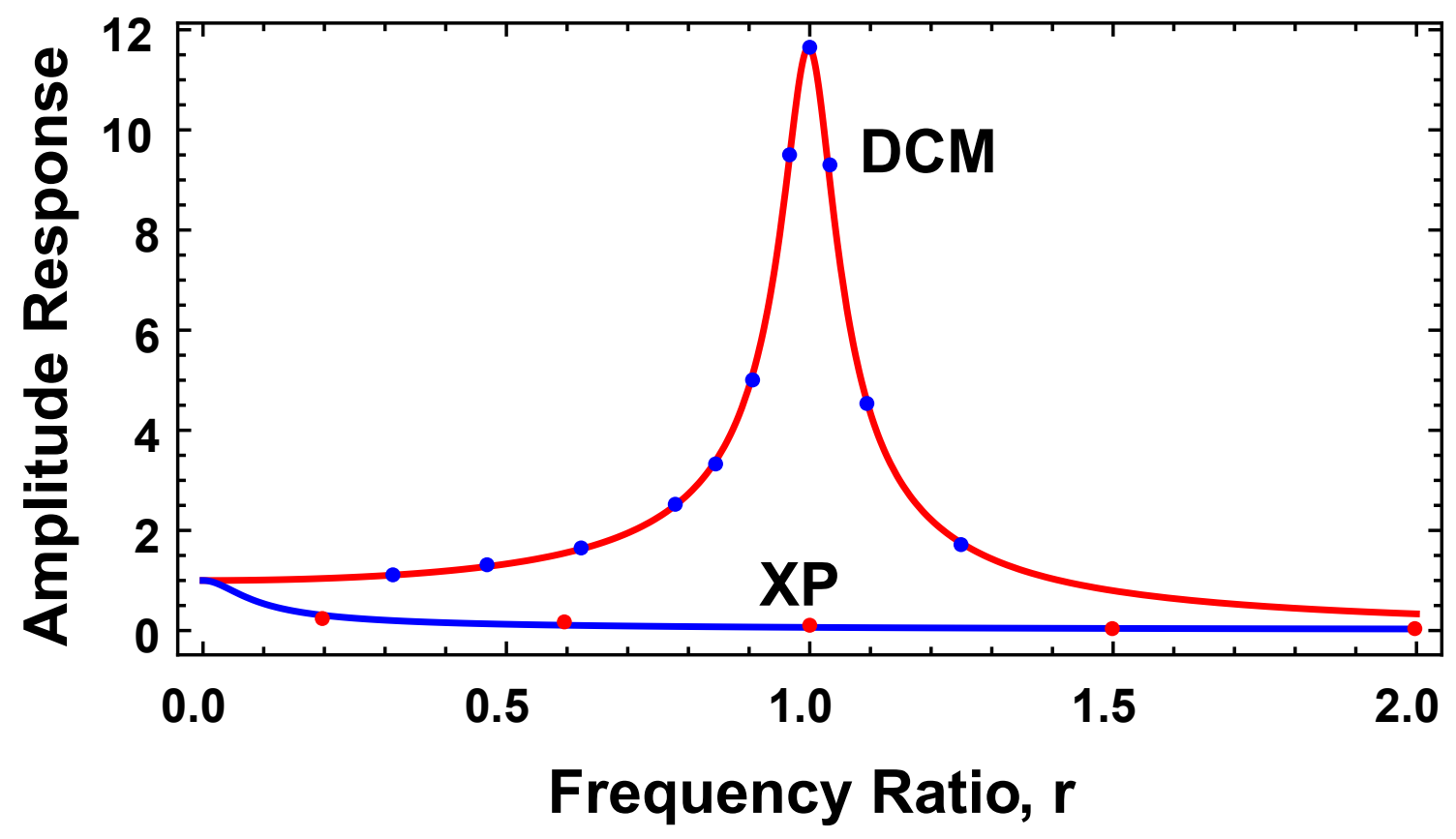

Figure 2.3: Theoretical curves and experimental data for amplitude vs. frequency response of the DCM and XP heads. Solid points represent experimental data from indentation tests in free space. 
magnitude depending on the manufacturer of the system.

The damping function is parabolic in shape with a minimum which has been manufactured to be near the middle of the indenter's full range of travel $(60 \mu \mathrm{m})$. This implies that in order to extract coupled system/sample properties one must compute either: (a) damping properties as a function of indenter travel with the damping response characterized as a function of displacement or (b) the damping properties of the indentation system at a specific point in the range of travel coupled with an analysis of the system/sample interaction at that point in space. Characterizing the damping response of the indentation system as a function of indenter displacement is possible, albeit time consuming. The testing protocols of the indentation system, specifically the surface find, dictate that tests begin in approximately the same location in the indenter's range of travel. With this in mind an examination of the system response just prior to contact with the sample allows for determination of coupled system/sample properties, namely stiffness and damping.

The equation of motion for the system remains unchanged at the moment the indenter and device come into contact, provided one accounts for the change in stiffness while neglecting the effective mass of the specimen that is moving. A representative MEMS beam with length, width and height of 500, 30 and 1 micron respectively will have a mass of $10^{-10} \mathrm{~kg}$, five orders of magnitude smaller than the mass of the system and can thus be ignored in the analysis. Damping in the beam will also be neglected although damping due to the tip/surface interaction is retained. In terms of testing free-standing beams presented in this work, the damping contribution from the beam is zero. Material damping is generally tested using experiments where the sample is monitored remotely because physical interactions with samples quickly wash out material effects. Effects of contact between the sample and indenter tip are shown to be negligible in the experimental results presented below. In order to present a full analysis, damping in due to the indenter/sample contact is included. While it will be denoted as contact damping, it could be analyzed as contact 


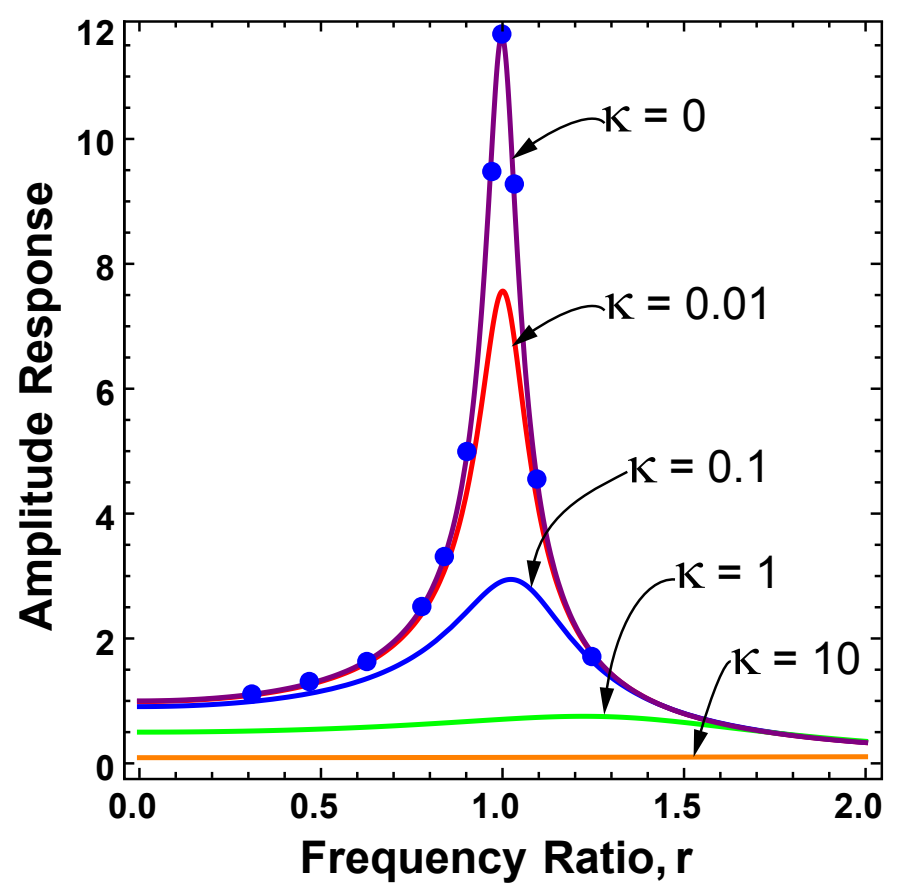

Figure 2.4: Amplitude response for the indenter in free space (purple) and in contact with differing values of $\kappa$ while $\eta$ is set to be zero. $\kappa=0$ represents the case where the indenter is oscillating in free space.

damping, intrinsic material damping or some combination of both depending on the material or structure in question. Typically, the stiffness of the frame is very large compared to the specimen stiffness which dictates that it can be ignored. A quick examination of contact mechanics shows the contact stiffness will grow quickly even for ultra-low loads (see above discussion of quasi-static response), allowing it to be disregarded in the new stiffness formulation. After contact, the stiffness can be described as

$$
k=k_{s}+k_{i}
$$

and the damping as

$$
D=D_{c}+D_{i}
$$

where $D_{c}$ is the damping coefficient associated with the tip/surface interface (contact), and $D_{i}$ is the damping coefficient of the indenter in free space. 
Repeating the above analysis, and normalizing the results, one obtains the amplitude ratio for the coupled system of the indenter and device:

$$
|\bar{H}(i \omega)|=\left|\frac{k X}{F_{0}}\right|=\frac{1}{\left[\left(1+\kappa-r^{2}\right)^{2}+(1+\eta)^{2}(2 \zeta r)^{2}\right]^{1 / 2}}
$$

The terms $\kappa$ and $\eta$ are the ratios of stiffness and damping shown below. To avoid confusion, the damping ratio of the system, $\zeta$, will be explicitly referred to as the system damping ratio where the ratio of sample stiffness to system stiffness will simply be referred to as the damping ratio.

$$
\kappa=\frac{k_{s}}{k_{i}} ; \quad \eta=\frac{D_{c}}{D_{i}}
$$

By inspection it is clear that eqn 2.14 reduces to eqn. 2.10 when $\kappa$ and $\eta$ are zero, i.e. the indenter is in free space. Figure 2.4 shows the sensitivity of the oscillation amplitude to contact with structures with stiffness of varying magnitude in comparison to that of the indenter springs. The expected shift in natural frequency due to the addition of additional stiffness is just begun to be seen in the $\kappa=0.1$ case in fig. 2.4. It appears that the change of the amplitude response of the oscillation is more sensitive to contact than the shift in natural frequency. In order to look closer at the relative amplitude and frequency shifts, eqn. 2.14 solved for the maximum value of $\mathrm{r}$ yields normalized amplitude response as well as normalized frequency response. Figure 2.5 clearly shows the amplitude response is more sensitive than the shift in natural frequency for small values of $\kappa$.

The fractional change in harmonic displacement associated with contact is now defined as:

$$
\frac{\Delta x_{c}}{\Delta x}=1-\frac{\left[\left(1-r^{2}\right)^{2}+(2 \zeta r)^{2}\right]^{1 / 2}}{\left[\left(1+\kappa-r^{2}\right)^{2}+(1+\eta)^{2}(2 \zeta r)^{2}\right]^{1 / 2}},
$$

where $\Delta x$ and $\Delta x_{c}$ are respectively the harmonic oscillation height in free space and after contact with the beam/sample. 


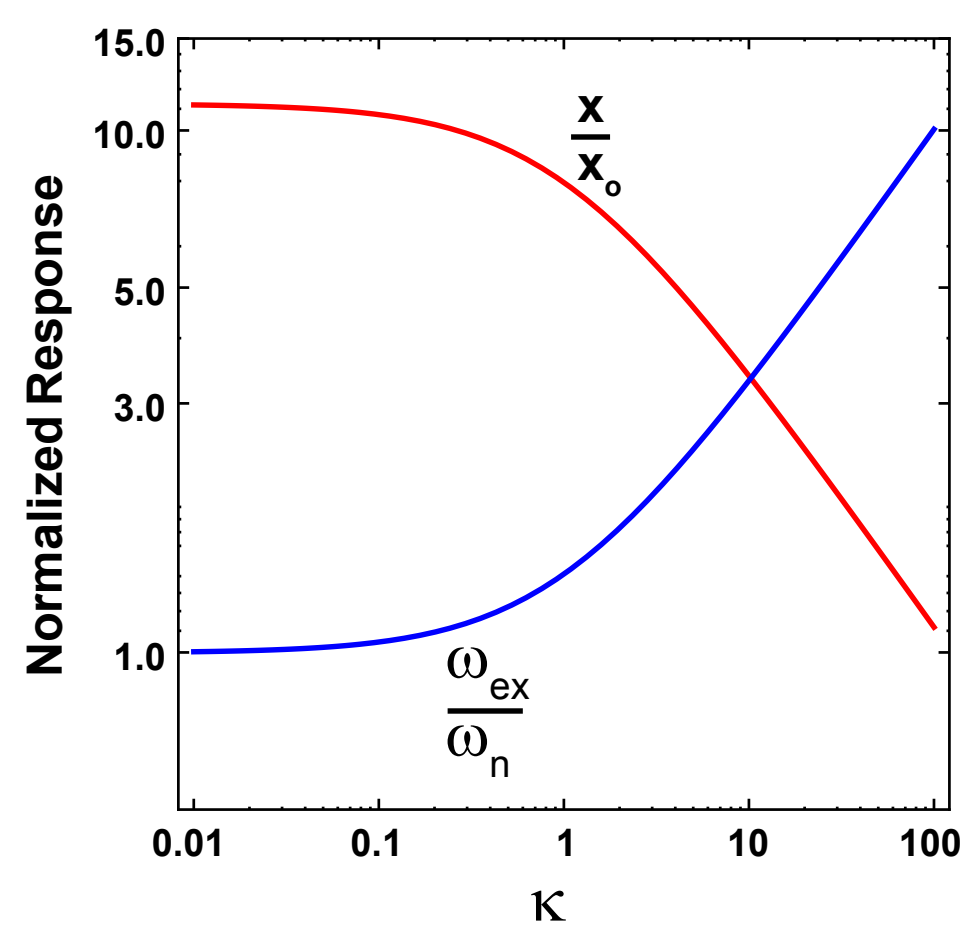

Figure 2.5: Amplitude and frequency response for various values of $\kappa$. The shift in amplitude response is more pronounced at low values of $\kappa$ than is the frequency shift.

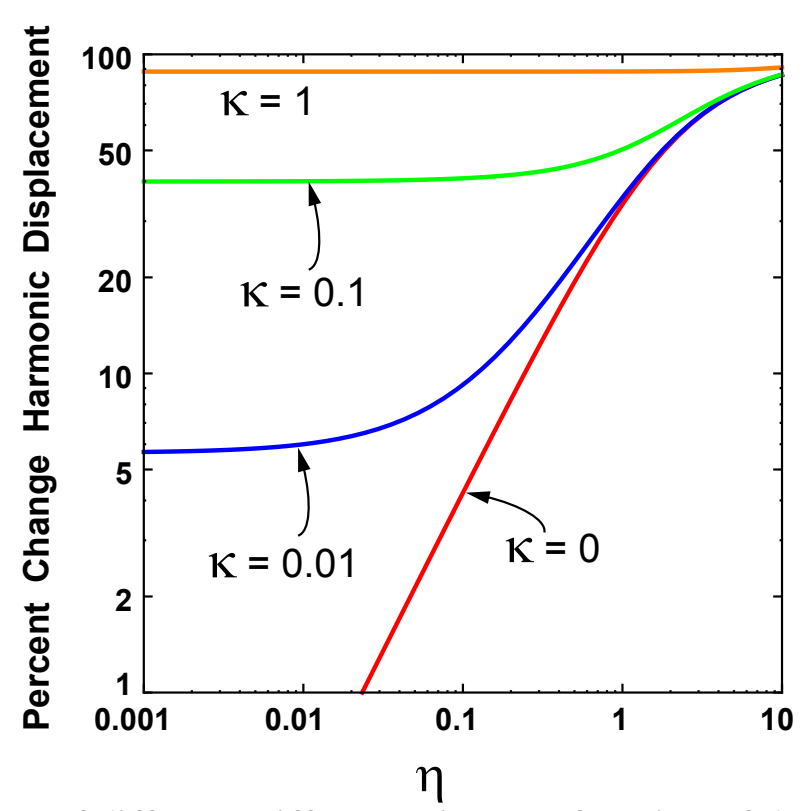

Figure 2.6: The effects of different stiffness ratios as a function of the damping ratio on the percent change in harmonic displacement upon establishing contact with $r=0.95$. The $\kappa$ $=0$ curve implies a structure with zero stiffness can be detected if damping of the order of magnitude of that of the indentation system is present. It is also clear that structures with stiffness on the order of $1 \mathrm{~N} / \mathrm{m}$ provide a significant response, regardless of the level of damping present. 


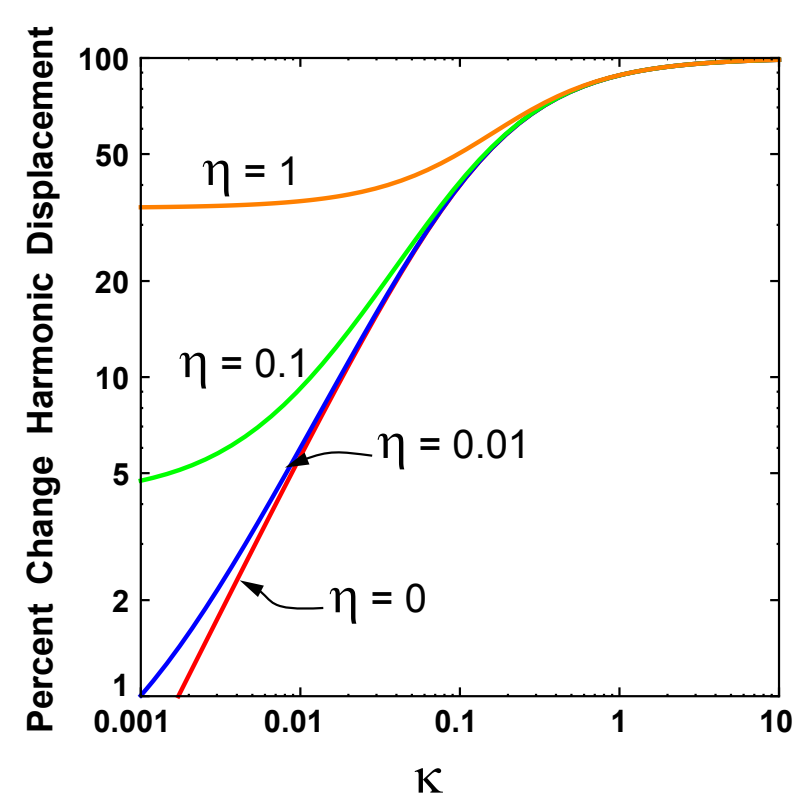

Figure 2.7: The effects of different damping ratios as a function of the stiffness ratio on the percent change in harmonic displacement upon establishing contact with $r=0.95$. The red curve $(\eta=0)$ is indicative of the response of thin metallic beams where material and contact damping are negligible.

This is the key result that can be used to assess the sensitivity of the harmonic displacement with regards to the instance of contact in order to identify the limits of system performance as a function of system and sample characteristics. Examining the amplitude response in terms of a percentage change in oscillation height yields a result that identifies contact as a deviation from zero, where zero corresponds to the indenter oscillating in free space. It should be noted that for the following analyses the mass, stiffness and damping are taken as those calculated from the analysis of experimental data in figure 2.3 unless noted differently.

Even though it will be shown that for experiments on metal beams there is no appreciable damping from material properties or tip/sample interaction, it is still interesting to examine cases where this is not true to yield insight into design of experiments for samples and structures where this may not be the case. The inclusion of any amount of sample damping increases the sensitivity of the analysis. As such these analyses present the lowest threshold for detection which will later be used with respect to testing of metallic beams. 


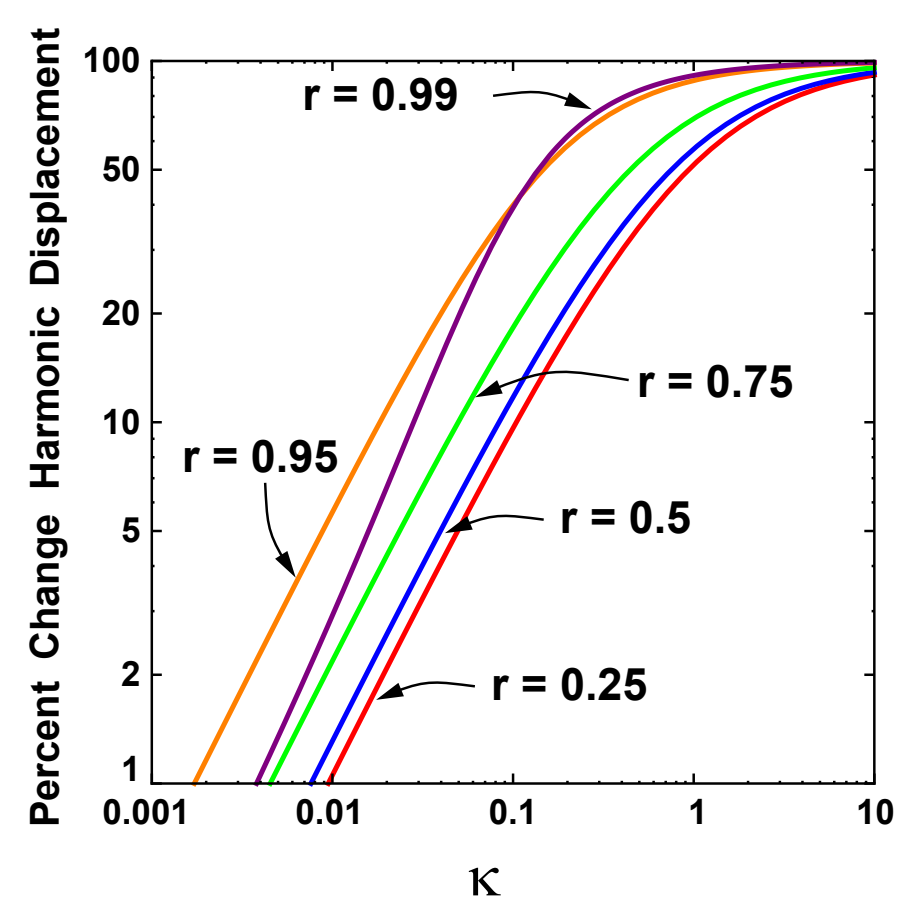

Figure 2.8: Percent change for a few values of $\mathrm{r}$. As expected the system becomes more sensitive to contact as the excitation frequency approaches the natural frequency of the system $(r=1)$ except at low stiffnesses for operating frequencies just under the natural frequency.

Examining figure 2.6, a couple important observations are immediately apparent: (i) detection of contact for samples with zero stiffness is still possible if an amount of damping on the order of magnitude of the indenter is present and (ii) beams with stiffness ratios as low as $\kappa=0.01$ will exhibit a significant change in the amplitude response even with no damping present. Figure 4.5 shows response to contact for varying levels of damping ratios. The case where $\eta=0$ is representative of testing on metallic beams.

It is natural to assume the oscillation frequency should be set as close to the system's natural frequency as possible to take advantage of the dynamic characteristics of the system. This assumption holds true until the stiffness of the beam becomes small in comparison to the stiffness of the indenter. Figure 2.8 clearly shows a decrease in response to contact for $r=0.99$ compared to $r=0.95$. When the operating frequency becomes very close to the natural frequency (i.e. the $r=0.99$ case), the only response seen is the decrease in ampli- 


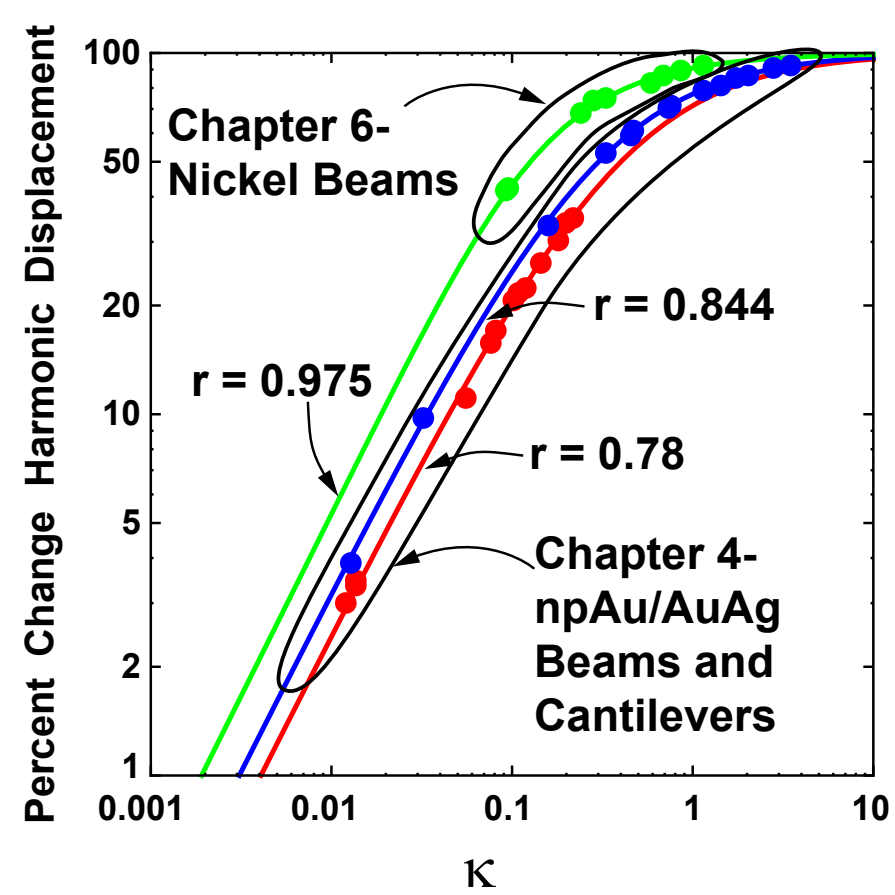

Figure 2.9: Data from nickel, AuAg and npAu beams and cantilevers showing good agreement between experimental data and theory using system parameters from the tests with eqn. 2.16 .

tude due to the difference in stiffness. The shift of the curve to the right (i.e. increase in natural frequency) to increase the response can only be taken advantage of in cases where the operating frequency is on the "down slope" of the frequency response curve as opposed to on top of it. Operating at, or very close to, a frequency ratio of $r=0.95$ ends up providing a maximum response in cases where samples are very compliant. Experimental data in figure 2.9 shows the percent change for various beams and cantilevers presented in the chapters to follow. This data confirms that damping is negligible as the curves in figure 2.9 are for $\eta=0$. The nickel beams used a $50 \mu \mathrm{m}$ sapphire spherical tip while the npAu and $\mathrm{AuAg}$ beams used a Berkovich diamond with a tip radius of $\sim 100 \mathrm{~nm}$.

For completeness, the effects of varying the system damping ratio are shown in figure 2.10. As the system damping ratio is a function of the hardware in a particular system, this figure might be useful to compare the sensitivity of instruments prior to purchase. Clearly, reducing the system damping ratio leads to larger response due to contact. There is likely 


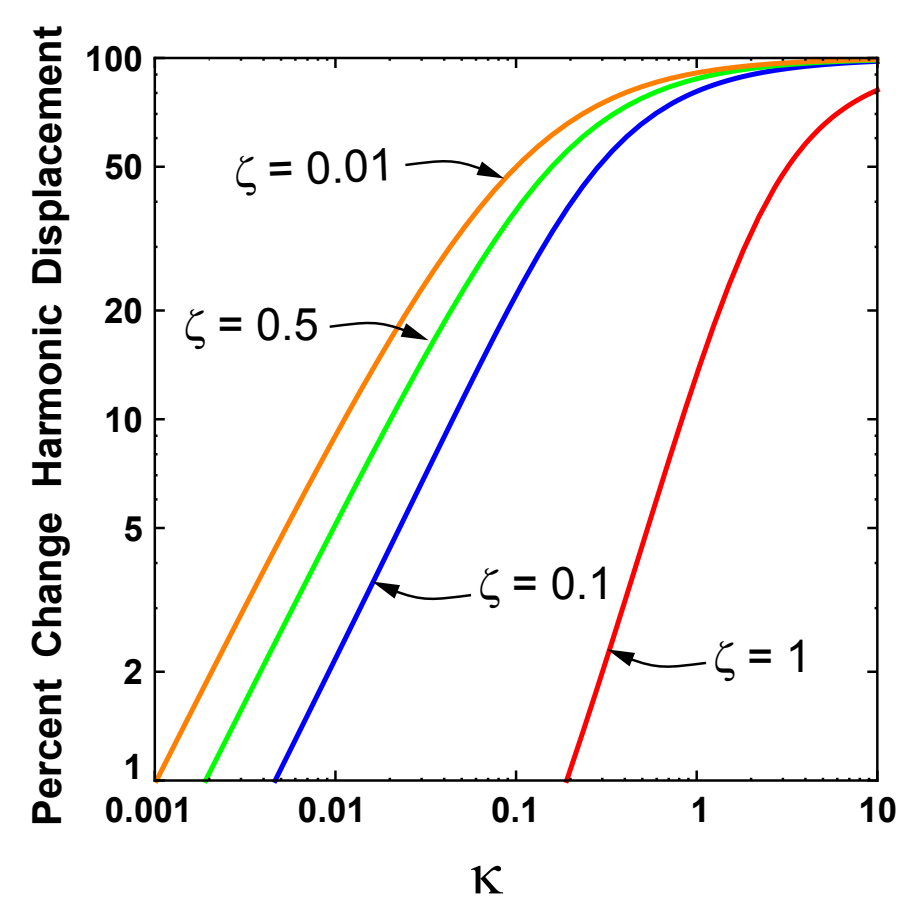

Figure 2.10: Effects of varying system damping ratio on sensitivity to contact. The indentation system at UVa has system damping ratio of approximately $\zeta=0.043$, similar to the green curve.

a trade-off involved with lowering the stiffness ratio such that it either becomes technologically impossible or monetarily unfeasible to produce instruments with lower system damping ratios. Although this would be an interesting problem to investigate, it is beyond the current scope of this work and will be ignored other than the presentation of figure 2.10 .

\subsection{Practical limitations}

It is useful to examine the detection limits of this analysis with regard to experimental limitations. The lower limit for detection is governed by environmental noise. Unless explicitly referenced, the system damping ratio and system stiffness in all following analyses and figures is assumed to be that of the system at UVa $\left(\zeta=0.043, k_{i}=82 \frac{\mathrm{N}}{\mathrm{m}}\right)$. In order to theoretically examine the effects of noise on the system response, it is assumed that the noise presents itself as a harmonic error. Figure 2.11 shows the error associated with varying noise/control error as a function of system properties by rearranging eqn. 2.10. Figure 


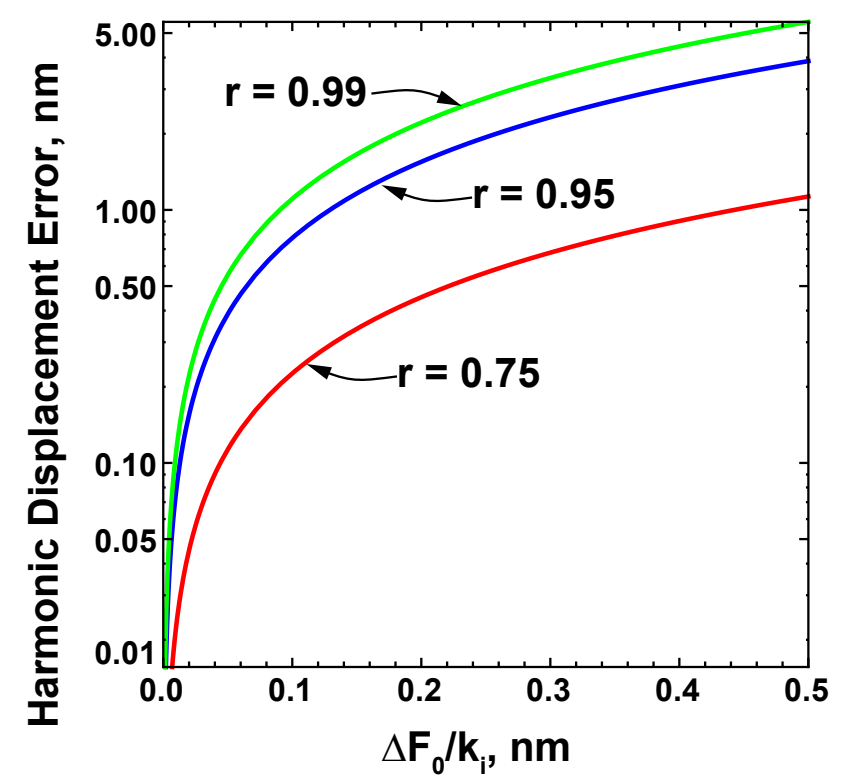

Figure 2.11: Harmonic displacement error as a function of system properties and error in harmonic load for $\zeta=0.043$. Operating closer to the natural frequency for the same error in harmonic load (i.e. noise/control error) increases the error in harmonic displacement.

2.11 can be used to determine the lower limit of detection via percent change oscillation by simply dividing half the harmonic displacement error by the harmonic displacement. This lower limit, used in conjunction with figure 2.8, provides an experimental framework for testing of compliant structures.

In order to investigate the assumption that error in the signal presents itself as a harmonic force error, the indenter was oscillated in free space at a number of frequencies and oscillation heights (10, 20 and 50nm). The harmonic displacement signals were examined and the maximum and minimum of the harmonic displacement were determined over a 30 second hold segment. This value was taken as the error in harmonic displacement and values for each frequency were averaged. Figure 2.12 is the compilation of these results, which are fit to eqn. 2.10 yielding a harmonic load error of $\sim 17 \mathrm{nN}$. This load error can be used to determine the harmonic displacement error at any operating frequency. The harmonic displacement errors in fig. 2.11 should be halved when calculating a percent change detection limit as half of the error presents above the prescribed harmonic displacement and half below. 


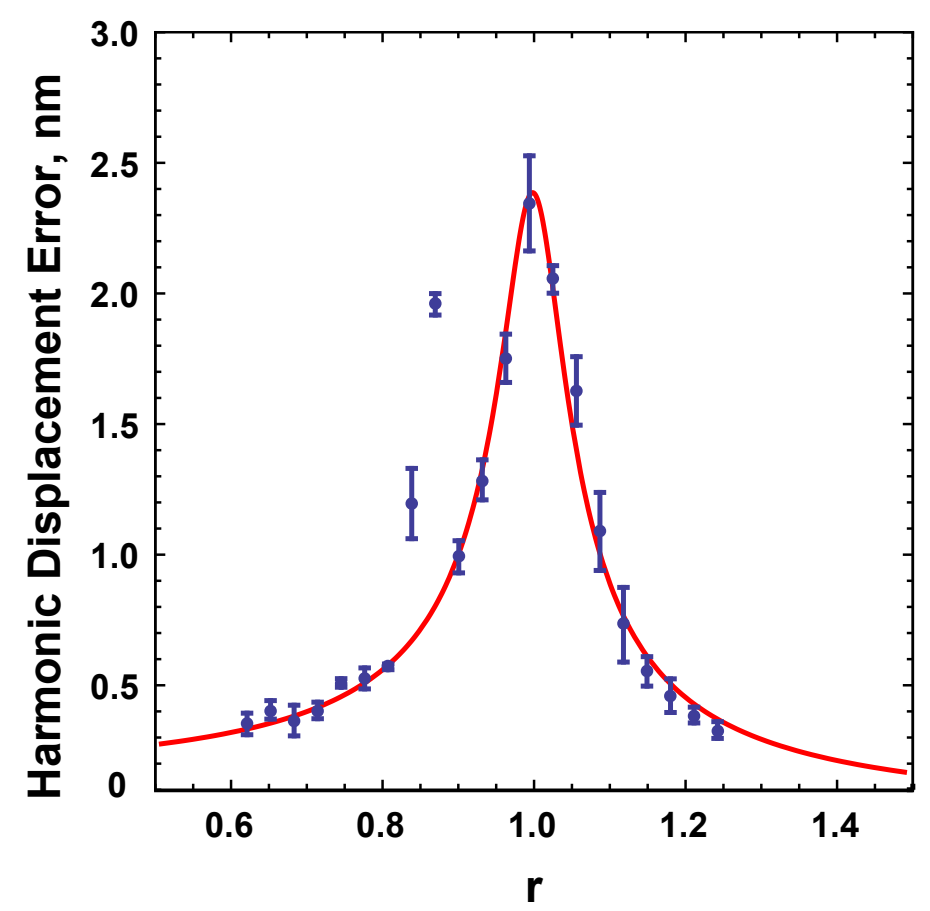

Figure 2.12: Least squares fit to the error in harmonic displacement. The fit produces a harmonic load of $\sim 17 \mathrm{nN}$ which is the assumed environmental noise in the system. It should be noted that the harmonic displacement error in the tests near $r=0.85$ is not included in the analysis. The "noise" in the displacement signal at these frequencies has an oscillatory component of $\omega=2 \mathrm{~Hz}$, implying the noise is coupled to an environmental vibration.

It should be noted the two outliers around $r=0.85(135$ and $140 \mathrm{~Hz})$ are not included in this fitting procedure. Upon close examination of their raw signals there is an oscillatory component to the noise of $\omega=2 \mathrm{~Hz}$. This oscillation is very pronounced at $\omega=140 \mathrm{~Hz}$ and less so at $\omega=135 \mathrm{~Hz}$. This coupled oscillation is likely due to mechanical vibrations in the surrounding building (air conditioning, fume hood fans, mechanical pumps, etc.) which are not removed by the vibration oscillation table. It should be noted that the indenter is currently on the second floor of a building and these oscillation couplings did not present themselves when it was in a laboratory which was on concrete slab. Even though this response is not ideal, by accurately knowing the dynamic response of our individual system we can avoid these operating frequencies or adjust testing parameters such that the noise is sufficiently reduced. 


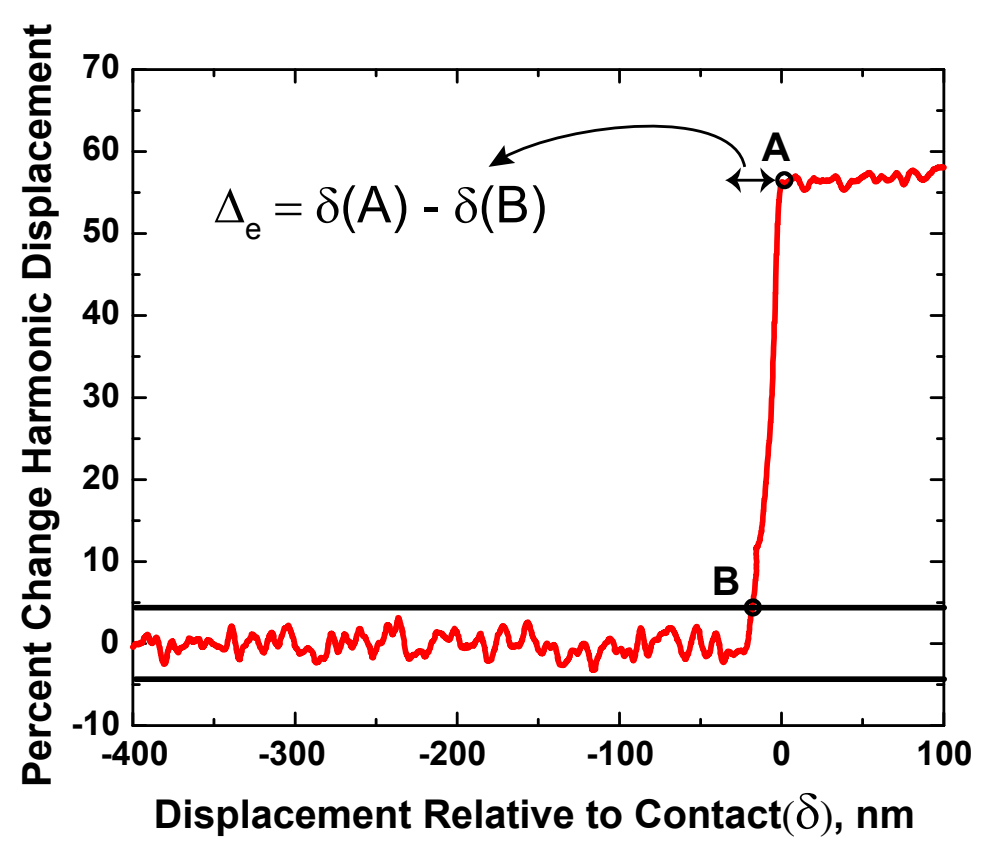

Figure 2.13: Experimental data on a $232 \mathrm{~nm}$ thick, $20 \mu \mathrm{m}$ wide by $500 \mu \mathrm{m}$ long nickel beam showing contact between sample and oscillatory component $(\mathrm{B})$ and point where the sample is fully loaded (A). The transition regime between points A and B is $\sim 20 \mathrm{~nm}$ which is also the harmonic oscillation amplitude for this test.

At this point it is important to distinguish the difference between the moment where the indenter contacts the sample due to the oscillatory component, point B in figure 2.13, and the point where the sample and indenter are fully in contact, point A, and is being loaded by the ramp loading component. In the case shown here the harmonic oscillation is $20 \mathrm{~nm}$ and the difference between point A and point B is $\sim 20 \mathrm{~nm}$. This loading regime (i.e. the distance between point A and B) is consistently on the order of the oscillation height in experiments and will be taken as the oscillation height in future discussion of error associated with the difference between these points. Point A is determined post test and is consistently determined within $\pm 1 \mathrm{~nm}$, regardless of oscillation height. Figure 2.14 shows that the load deflection response is not affected by either approach rate or varying oscillation heights.

Experimentally, point $\mathrm{B}$ is the user defined percent change harmonic displacement which determines the start of the test. This contact definition, chosen above the noise in the signal prior to contact as discussed above, is shown as the solid line intersecting point B in 


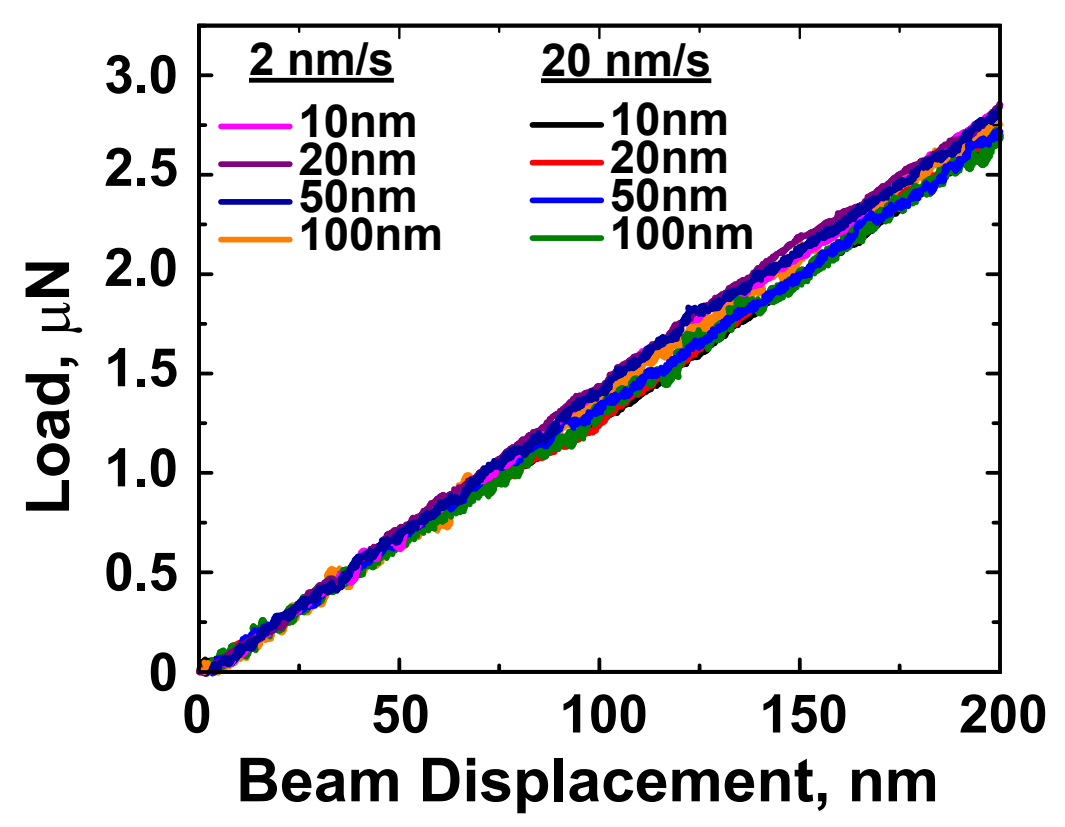

Figure 2.14: Experimental data on a $232 \mathrm{~nm}$ thick, $20 \mu \mathrm{m}$ wide by $500 \mu \mathrm{m}$ long nickel beam showing load-displacement response for different oscillation heights and approach speeds. Response is identical showing there is no adverse effect from large harmonic oscillations or varying approach speed.

figure 2.13 . It is important to note that experimentally the difference between points $\mathrm{A}$ and B can be drastically reduced if the stiffness of the beam is known via experiments or can be approximated. In this case the "trigger" where the test is started can be moved to lessen the difference. In figure 2.13 this might mean the solid line, and subsequently the contact point $\mathrm{B}$, could be moved up to $\sim 50 \%$ if the beam is known to have a stiffness that will produce a percent change harmonic displacement greater than $50 \%$. Clearly as the stiffness of the sample decreases, the change in harmonic displacement due to contact decreases and the identification of contact becomes more difficult. It is in these scenarios that the method described above is particularly useful. For stiffer samples the harmonic oscillation can be significantly reduced (usually down to $1 \mathrm{~nm}$ ) such that there is essentially no "loading regime" and point A and point B are the same. Additionally, accurate determination of the point of contact is important where loading schemes are time or space dependent as will be described below. 


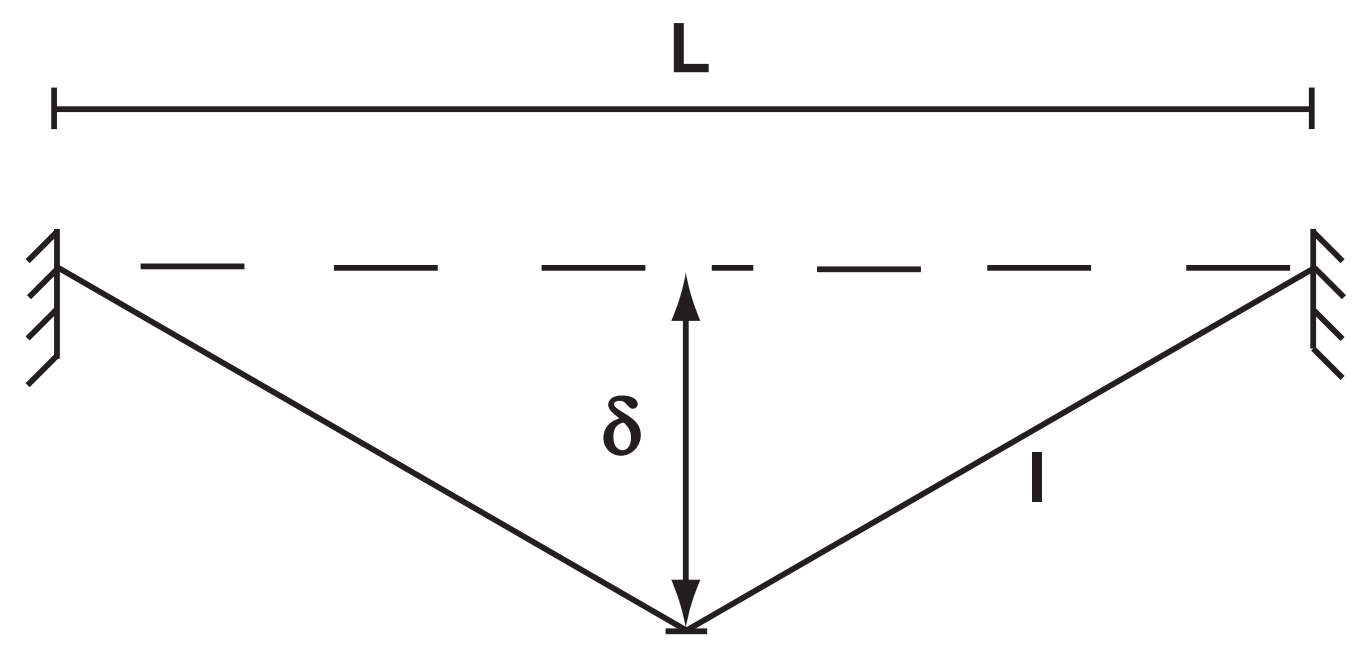

Figure 2.15: Representative diagram of stretch dominated MDE test.

\subsection{Constant strain rate testing}

A criticism of MDE tests (via indentation) is the inability to test at constant strain rate. Most existing work is tested with constant velocity which results in testing a wide range of strain rates over the course of the test. Depending on the test these strain rates can vary as much as three orders of magnitude. The most common method used to account for strain rate is to present the strain rate at fracture [12, 19]. In order to more fully characterize the mechanical response of beams, derivations for constant strain rate testing and the experimental procedures necessary to implement them are provided below.

For simplicity, assume the system is dominated by stretching behavior. Figure 3.6 shows the relevant beam length, $\mathrm{L}$, and the displacement experienced by the beam, $\delta$. Geometry dictates that:

$$
l=\sqrt{\left(\frac{L}{2}\right)^{2}+\delta^{2}}
$$

where $l$ is the half-length of the deformed beam. Knowing the change in length of the beam divided by the original length is equal to the strain, $\varepsilon$, allows us to compute strain as a function of beam length and displacement. 


$$
\varepsilon=\sqrt{4\left(\frac{\delta}{L}\right)^{2}+1}-1
$$

Taking the time derivative of equation 2.18 yields:

$$
\dot{\varepsilon}=\frac{4 \delta \cdot \dot{\delta}}{L^{2} \sqrt{1+\frac{4 \delta^{2}}{L^{2}}}}
$$

where $\dot{\delta}$ is the displacement rate of the beam and $\dot{\varepsilon}$ is the imposed strain rate. Equation 2.19 can easily be rearranged such that displacement rate is solely a function of beam length (constant), strain rate (constant) and displacement:

$$
\dot{\delta}=\frac{\dot{\varepsilon} \cdot L^{2} \sqrt{1+\frac{4 \delta^{2}}{L^{2}}}}{4 \delta} .
$$

This is the result used to achieve constant strain rate in experiments. A discussion of the effect of the uncertainty in displacement due to the harmonic oscillation on this equation (the difference between points $\mathrm{A}$ and $\mathrm{B}$ in figure 2.13) first requires a look at testing protocols necessary to experimentally enact constant strain rate testing of beams. Examining eqn. 2.20 it becomes clear that small displacements will result in large displacement rates which become smaller as displacement increases. Experimentally large displacement rates are problematic in that displacement is monitored via the change in capacitance and a given displacement is only achieved via the load feedback loop. A major shortcoming of the UVa indentation system, and from experience the Hysitron indentation system as well, is the inability to directly manipulate the control system or the raw voltage and capacitance signals. In this regard experiments are designed to avoid relatively small displacements such that the system responds in a stable fashion to the requested displacements and displacement rates.

In order to avoid large displacement rates, the displacement rate is linearly ramped 

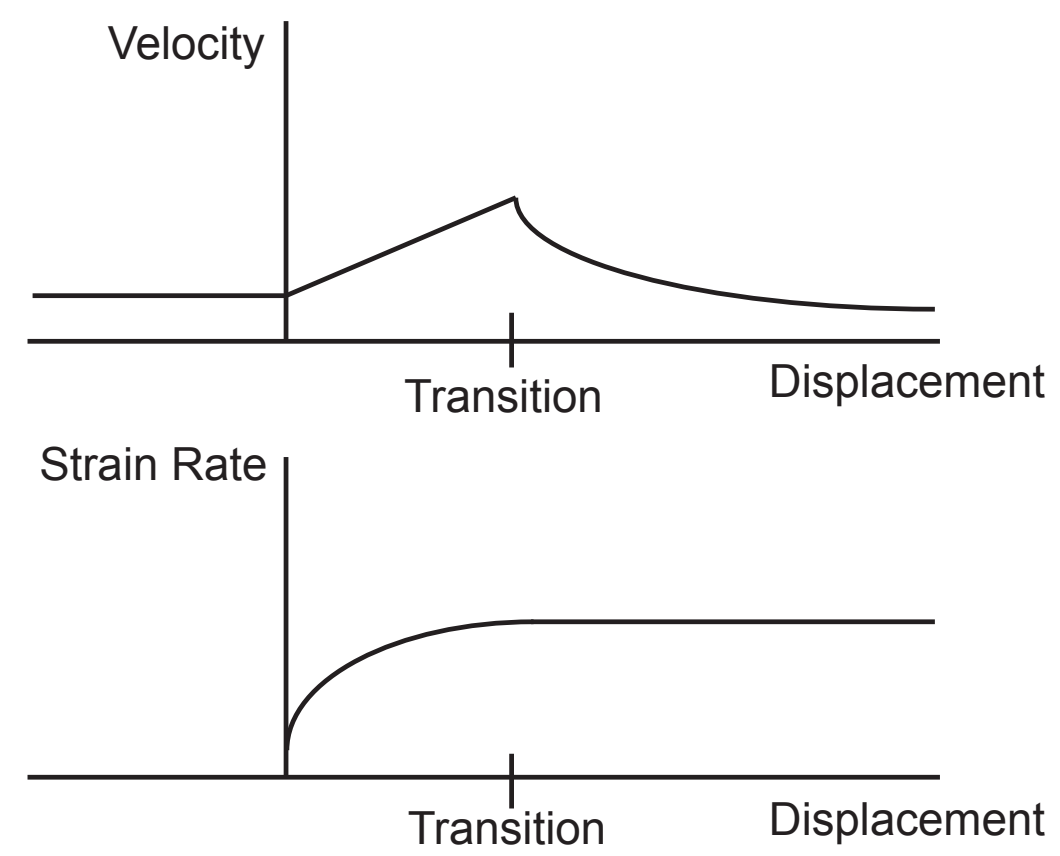

Figure 2.16: Representative velocity and strain rate profiles which ramp the velocity up to a transition point where the constant strain rate loading is then enacted.

up from the approach loading rate to a transition displacement/strain where the velocity function is then defined as eqn 2.20 . The velocity and strain rate profiles as a function of displacement are seen in figure 2.16. The transition point is chosen to be in the linear elastic regime because material properties are accepted to be rate insensitive in this regime.

We can now examine the error associated with the regime between points A and B in figure 2.13, which will be referred to as $\Delta_{e}$. By expressing the displacement term in eqn. 2.20 as $\left(\delta-\Delta_{e}\right)$, the effects of the regime where the indenter is coming into contact with the beam on the displacement rate can be captured. The $\Delta_{e}$ terms in the numerator can be ignored as the expanded terms involving $\Delta_{e}$ will be small relative to the $\delta^{2}$ term. Additionally the entire square root term is essentially unity for cases where $\delta<20 L$, further minimizing any contribution from the $\Delta_{e}$ term in the numerator. Equation 2.20 becomes:

$$
\dot{\delta}=\frac{\dot{\varepsilon}_{t} \cdot L^{2} \sqrt{1+\frac{4 \delta^{2}}{L^{2}}}}{4\left(\delta-\Delta_{e}\right)}
$$




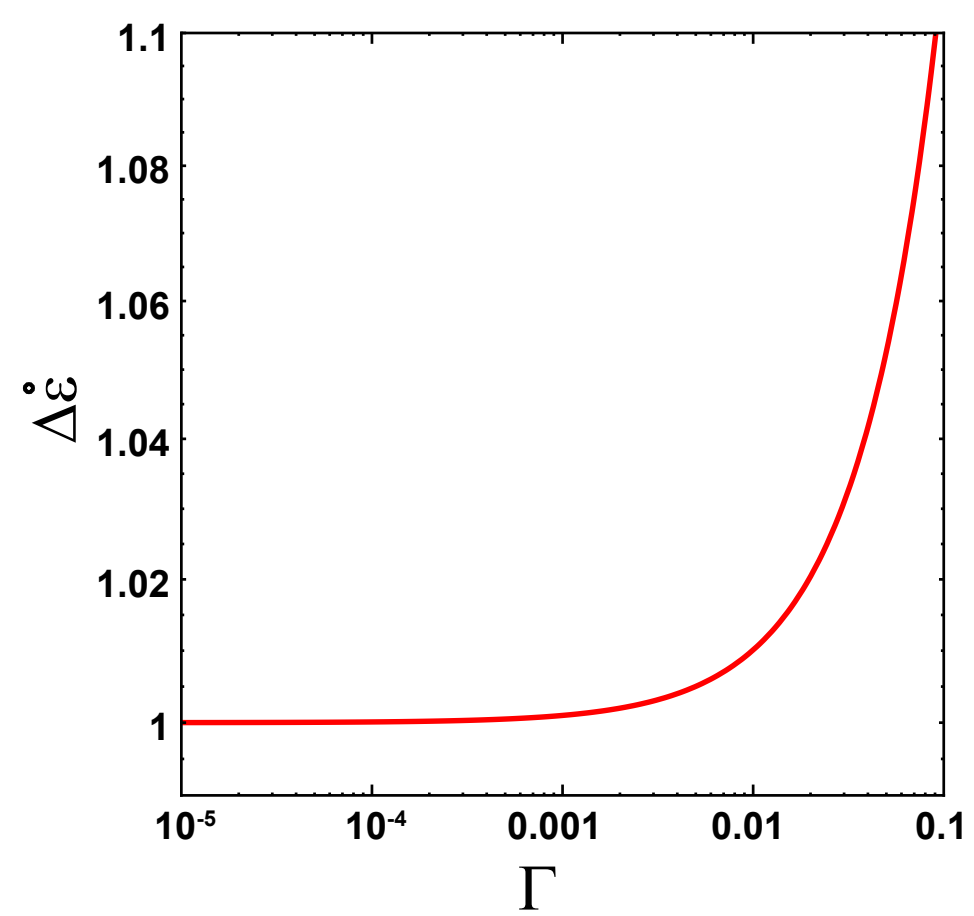

Figure 2.17: Difference in target strain rate versus that seen experimentally as a function of contact error and beam displacement.

where $\dot{\varepsilon}_{t}$ is the target strain rate which is used to calculate the displacement rate. Plugging eqn. 2.21 into eqn. 2.19 yields:

$$
\dot{\varepsilon}_{a}=\frac{\dot{\varepsilon}_{t} \delta}{\left(\delta-\Delta_{e}\right)}
$$

where $\dot{\varepsilon}_{a}$ is the actual strain rate experienced by the beam during testing. This result in terms of normalized variables is:

$$
\Delta \dot{\varepsilon}=\frac{1}{(1-\Gamma)}
$$

where $\Gamma$ is the ratio of the missed contact, $\Delta_{e}$, to the displacement of the beam, $\delta$ and $\Delta \dot{\varepsilon}$ is the ratio of the actual strain rate experienced by the beam to the target strain rate. Figure 2.17 shows the effects of the contact error on strain rate. Clearly there is little effect on the experimental strain rate when displacement is large or the harmonic oscillation, and associated error in contact, is large. This result suggests that harmonic displacement should be kept to a minimum while still allowing for contact definition in order to minimize the 
error in strain rates over the course of the test. Additionally it is interesting to note the error in achieved strain rate goes down as beam displacement increases which is ideal as the effects of differences will be more pronounced at large deflections/strains where plastic deformation mechanisms are active. 


\section{Chapter 3}

\section{Test Interpretation}

\subsection{Point loading fixed-fixed beams}

The following analysis will examine the full non-linear load-deflection response of elastic beams with tensile residual stress and identify the relevant asymptotic limits corresponding to classical types of response. Design maps are constructed to illustrate critical values of load and displacement that identify regimes where these limits are accurate, using dimensionless parameters involving pre-stretch, modulus and dimensions. These maps can, and are in following chapters, be used to identify appropriate geometries, loads and deflections to target modulus and/or residual stress. Further, a simple closed-form expression is identified for non-linear responses that facilitate property extraction when asymptotic expressions are not valid. A critical contribution is the development of an explicit analytical relationship for load-deflection response which avoids the complication of using implicit solutions, which requires non-linear root-finding to determine the mechanical stretch in the beam.

\subsubsection{Full solution and an effective approximation}

Consider a beam of length $L$, clamped at both ends and loaded at its center with the point force $P$. Assuming plane sections remain plane and that the beam is slender enough 

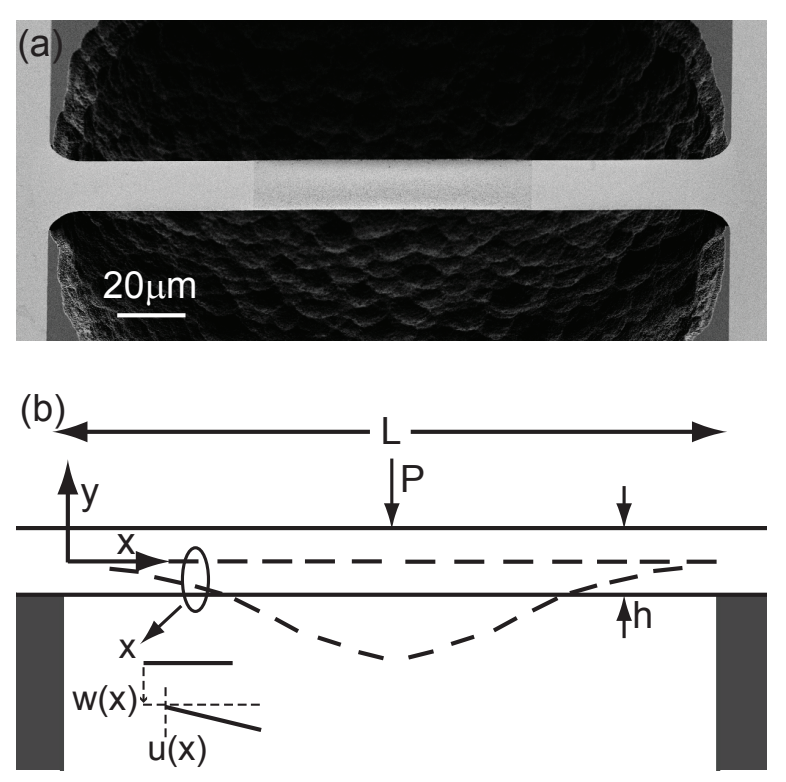

Figure 3.1: (a) SEM of representative MEMS beam used in point-load test. (b) Side view of coordinates and deformation variables used in the analysis.

to ignore transverse shear, and allowing for moderate rotations, the total axial strain in the beam is given by:

$$
\varepsilon(x)=u^{\prime}(x)+\frac{1}{2}\left(w^{\prime}(x)\right)^{2}-w^{\prime \prime}(x) \cdot y
$$

where $y$ is the distance from the centerline, $u(x)$ is the axial displacement in the beam, and $w(x)$ is the transverse displacement. The stress in the beam is given by:

$$
\sigma(x)=E \varepsilon(x)+\sigma_{R}=E\left[\varepsilon(x)+\varepsilon_{R}\right]
$$

Let $\varepsilon_{R}$ be a positive pre-stretch in the beam: that is, $\varepsilon_{R}$ is the mechanical strain in the beam at zero elongation, caused by tensile residual stress created during fabrication. The principle of virtual work says:

$$
\delta W_{i n t}-\delta W_{e x t}=\int_{0}^{L / 2}\left(\int_{A} \sigma(x) \delta \varepsilon(x) d A\right) d x-\frac{P}{2} \delta w\left(x=\frac{L}{2}\right)=0
$$


The strain variation can be written in terms of variations in the displacements:

$$
\delta \varepsilon(x)=\delta u^{\prime}(x)+w(x) \delta w^{\prime}(x)-\delta w^{\prime \prime}(x) \cdot y
$$

The stress is equal to $\sigma(x)=E\left(\varepsilon(x)+\varepsilon_{R}\right)$, where $\mathrm{E}$ is the approximate modulus. Thus, the variational statement is:

$$
\begin{array}{r}
\delta W_{t o t}=\int_{0}^{L / 2}\left(\int_{A} E\left(\varepsilon(x)+\varepsilon_{R}\right)\left(\delta u^{\prime}(x)+w^{\prime}(x) \delta w^{\prime}(x)-\delta w^{\prime \prime}(x) \cdot y\right) d A\right) d x \\
-\frac{P}{2} \delta w\left(x=\frac{L}{2}\right)
\end{array}
$$

PVW says $\delta W_{\text {tot }}=0$. We will evaluate the integrals term by term and then collect them at the end. The first one is:

$$
\begin{array}{r}
\int_{0}^{L / 2}\left(\int_{A} E\left(u^{\prime}(x)+\frac{1}{2}\left(w^{\prime}(x)\right)^{2}-w^{\prime \prime}(x) \cdot y+\varepsilon_{R}\right) \delta u^{\prime}(x) d A\right) d x= \\
\int_{0}^{L / 2}\left(\int_{A} E\left(u^{\prime}(x)+\frac{1}{2}\left(w^{\prime}(x)\right)^{2}+\varepsilon_{R}\right) \delta u^{\prime}(x) d A\right) d x
\end{array}
$$

where the term with the $w^{\prime \prime}(x) \cdot y$ integrates to zero if the beam is symmetric about the center line. Integrating the right hand side by parts, one obtains:

$$
\begin{gathered}
\left.E A\left(u^{\prime}(x)+\frac{1}{2}\left[w^{\prime}(x)\right]^{2}+\varepsilon_{R}\right) \delta u(x)\right|_{0} ^{L / 2}- \\
E A \int_{0}^{L / 2}\left(u^{\prime}(x)+\frac{1}{2}\left[w^{\prime}(x)\right]^{2}+\varepsilon_{R}\right)^{\prime} \delta u(x) d x
\end{gathered}
$$

The second term is:

$$
\begin{array}{r}
\int_{0}^{L / 2}\left(\int_{A} E\left(u^{\prime}(x)+\frac{1}{2}\left(w^{\prime}(x)\right)^{2}-w^{\prime \prime}(x) \cdot y+\varepsilon_{R}\right) w^{\prime}(x) \delta w^{\prime}(x) d A\right) d x= \\
\int_{0}^{L / 2}\left(\int_{A} E\left(u^{\prime}(x)+\frac{1}{2}\left(w^{\prime}(x)\right)^{2}+\varepsilon_{R}\right) w^{\prime}(x) \delta w^{\prime}(x) d A\right) d x
\end{array}
$$


where again the term $w^{\prime \prime}(x) \cdot y$ integrates over the cross-sectional area to zero for a symmetric beam. Integration by parts yields:

$$
\begin{array}{r}
\int_{0}^{L / 2}\left(\int_{A} E\left(u^{\prime}(x)+\frac{1}{2}\left(w^{\prime}(x)\right)^{2}-w^{\prime \prime}(x) \cdot y+\varepsilon_{R}\right) \delta w^{\prime \prime}(x) \cdot y \cdot d A\right) d x \\
=E I \int_{0}^{L / 2} w^{\prime \prime}(x) \delta w^{\prime \prime}(x) d x
\end{array}
$$

Here, the first two terms that are integrated with respect to y, so they cancel. $I=\int_{A} y^{2} d A$. Integration by parts twice yields:

$$
\left.E I w^{\prime \prime}(x) \delta w^{\prime}(x)\right|_{0} ^{L / 2}-\left.E I w^{\prime \prime \prime}(x) \delta w(x)\right|_{0} ^{L / 2}+E I \int_{0}^{L / 2} w^{\prime \prime \prime \prime}(x) \delta w(x) d x
$$

Now we have to collect terms. The integrals that are left say:

$$
\begin{array}{r}
E A \int_{0}^{L / 2}\left(u^{\prime}(x)+\frac{1}{2}\left[w^{\prime}(x)\right]^{2}+\varepsilon_{R}\right)^{\prime} \delta u(x) d x=0 \\
\left(E I \int_{0}^{L / 2} w^{\prime \prime \prime \prime}(x)-E A \int_{0}^{L / 2}\left[\left(u^{\prime}(x)+\frac{1}{2}\left[w^{\prime}(x)\right]^{2}+\varepsilon_{R}\right) w^{\prime}(x)\right]^{\prime}\right) \delta w(x) d x=0
\end{array}
$$

where the second integral above picks up terms from the last two terms of the PVW expression. Both the integrands must be zero because the virtual displacement $\delta u(x)$ and $\delta w(x)$ are arbitrary. These equations become:

$$
\begin{array}{r}
E A\left(u^{\prime}(x)+\frac{1}{2}\left[w^{\prime}(x)\right]^{2}+\varepsilon_{R}\right)^{\prime}=0 \\
E I w^{\prime \prime \prime \prime}(x)-E A\left[\left(u^{\prime}(x)+\frac{1}{2}\left[w^{\prime}(x)\right]^{2}+\varepsilon_{R}\right) w^{\prime}(x)\right]^{\prime}=0
\end{array}
$$


Expanding the derivatives of the last term in the second equation by the chain rule, these equations become:

$$
\begin{array}{r}
E A\left(u^{\prime}(x)+\frac{1}{2}\left[w^{\prime}(x)\right]^{2}+\varepsilon_{R}\right)^{\prime}=0 \\
E I w^{\prime \prime \prime \prime \prime}(x)-E A\left[\left(u^{\prime}(x)+\frac{1}{2}\left[w^{\prime}(x)\right]^{2}+\varepsilon_{R}\right)\right]^{\prime} w(x) \\
-E A\left[\left(u^{\prime}(x)+\frac{1}{2}\left[w^{\prime}(x)\right]^{2}+\varepsilon_{R}\right)\right] w^{\prime \prime}(x)=0
\end{array}
$$

But the middle term is zero, according to 3.12 above. The equations now become:

$$
\begin{array}{r}
E A\left(u^{\prime}(x)+\frac{1}{2}\left[w^{\prime}(x)\right]^{2}+\varepsilon_{R}\right)^{\prime}=0 \\
E \operatorname{Iw}^{\prime \prime \prime \prime}(x)-E A\left[\left(u^{\prime}(x)+\frac{1}{2}\left[w^{\prime}(x)\right]^{2}+\varepsilon_{R}\right)\right] w^{\prime \prime}(x)=0
\end{array}
$$

Assuming plane stress (the results are identical for plane strain, provided one substitutes $(1+v) \varepsilon_{R}$ for $\varepsilon_{R}$ and $E /\left(1-v^{2}\right)$ for $E$.), the principle of virtual work and 3.15 yields the condition that:

$$
\left(u^{\prime}(x)+\frac{1}{2}\left[w^{\prime}(x)\right]^{2}+\varepsilon_{R}\right)=\text { constant }=\lambda^{2}
$$

where $\lambda$ is a solution variable to be computed. $\lambda^{2}$ represents the spatially uniform mechanical strain in the deformed state (i.e. that associated with the state created by applying the point load) along the neutral axis. Hence, $\lambda^{2}$ also represents the average stress via $\sigma=E \lambda^{2}$. Here, we consider only positive values of $\lambda^{2}$, i.e. tension in the beam. Hence, the first governing equation becomes:

$$
w^{\prime \prime \prime \prime}(x)-\frac{12 \lambda^{2}}{h^{2}} w^{\prime \prime}(x)=0
$$

The boundary conditions are obtained from the terms in the PVW statement where the variations are evaluated at the boundaries. Where the displacements are known, the variations are zero. The prescribed boundary conditions are $w(0)=0, w^{\prime}(0)=0, w^{\prime}(L / 2)=0, u(0)=$ 
0 , and $u(L / 2)=0$. The terms involving $\delta w(0), \delta w^{\prime}(0), \delta u(0), \delta u(L / 2)$ and $\delta w^{\prime}(L / 2)$ are zero. The remaining boundary condition (implied by the PVW statement) is:

$$
E I w^{\prime \prime \prime}\left(\frac{L}{2}\right)=-\frac{P}{2}
$$

which is simply a statement the the shear force at the centerline is equal to the applied load. In summary, the boundary conditions are:

$u(x=0)=u\left(x=\frac{L}{2}\right)=w(x=0)=w^{\prime}(x=0)=w^{\prime}\left(x=\frac{L}{2}\right)=0 ; \quad w^{\prime \prime \prime}\left(x=\frac{L}{2}\right)=-\frac{P}{2 E}(3.20)$

Thus we have four bc's for the fourth order differential equation given by 3.18 . However, the parameter $\lambda$ is an unknown, and we need another equation to completely specify the solution.

The last piece of the puzzle is that the axial displacement at the centerline should be zero at $x=\frac{L}{2}$ from symmetry. Integrating 3.17 to solve for $u(L / 2)=0$, one obtains:

$$
\lambda^{2}-\varepsilon_{R}-\frac{1}{L} \int_{0}^{L / 2}\left[w^{\prime}(x)\right]^{2} d x=0
$$

In 3.21, the first term represents the axial elongation due to the axial stress generated in the beam, the second term represents contraction due to cooling from elevated temperature, while the third term represents the out of plane stretching.

\subsubsection{Normalization}

A few simple normalizations will lead to simplified governing equations and solutions. Let $\bar{x}=\frac{x}{L}$, such that $\frac{d}{d x}=\frac{d}{d(L \bar{x})}=\frac{1}{L} \frac{d}{d \bar{x}}$ Let $\bar{w}=w / h$. Now 3.18 becomes:

$$
\bar{w}^{\prime \prime \prime \prime}(\bar{x})-\frac{12 \lambda^{2} L^{2}}{h^{2}} \bar{w}^{\prime \prime}(\bar{x})=0
$$


where the primes are now indicating derivatives with respect to $\bar{x}$. The final boundary condition in 3.20 becomes:

$$
\bar{w}^{\prime \prime \prime}\left(\frac{1}{2}\right)=-\frac{6 P L^{3}}{E b h^{4}}
$$

It is convenient to normalize the load by a reference load. For an unstressed beam with small deflections, the classical beam result predicts:

$$
w\left(\frac{L}{2}\right)=\frac{P L^{3}}{16 E b h^{3}}
$$

Thus, the load at which the classical deflection result equals one beam thickness is given by:

$$
P_{0}=\frac{16 E b h^{4}}{L^{3}}
$$

If we define $\bar{P}=P / P_{0}$, then:

$$
\frac{6 P L^{3}}{E b h^{4}}=96 \bar{P}
$$

Let $\bar{u}=2 u / L$. The other boundary conditions become:

$$
\bar{u}(0)=\bar{u}\left(\frac{1}{2}\right)=\bar{w}(0)=\bar{w}^{\prime}(0)=\bar{w}^{\prime}\left(\frac{1}{2}\right)=0
$$

3.21 becomes:

$$
\lambda^{2}-\varepsilon_{R}-\left(\frac{h}{L}\right)^{2} \int_{0}^{1 / 2}\left[\bar{w}^{\prime}(\bar{x})^{2}\right] d \bar{x}=0
$$

We can see the if we let $\Lambda=\sqrt{12} \lambda L / h$ and $\overline{\varepsilon_{R}}=12 \varepsilon_{R} L^{2} / h^{2}$, we can remove $h / L$ completely from the equations, yielding:

$$
\begin{array}{r}
\bar{w}^{\prime \prime \prime \prime}(\bar{x})-\Lambda^{2} \bar{w}^{\prime \prime}(\bar{x})=0 \\
\Lambda^{2}-\overline{\varepsilon_{R}}-12 \int_{0}^{1 / 2}\left[\bar{w}^{\prime}(\bar{x})\right]^{2} d \bar{x}=0 \\
\bar{w}(0)=\bar{w}^{\prime}(0)=\bar{w}^{\prime}\left(\frac{1}{2}\right)=0 ; \quad \bar{w}^{\prime \prime \prime}\left(\frac{1}{2}\right)=96 \bar{P}
\end{array}
$$




\subsubsection{Solutions}

The solution to the governing equations is given by:

$$
\bar{w}(\bar{x})=\frac{96 \bar{P}\left(\Lambda \bar{x}-\sinh \Lambda \bar{x}+(\cosh \Lambda \bar{x}-1) \tanh \frac{\Lambda}{4}\right)}{\Lambda^{3}}
$$

where the mechanical strain in the beam in the deformed state is given by the solution to

$$
\Lambda^{2}-\bar{\varepsilon}_{R}=12 \Delta^{2} \cdot F(\Lambda)
$$

where $\Delta=\bar{w}(1 / 2)$ is the deflection of the load point, and

$$
F(\Lambda)=\frac{\Lambda\left(\operatorname{sech} \frac{\Lambda}{4}\right)^{2}\left(2 \Lambda+\Lambda \cosh \frac{\Lambda}{2}-6 \sinh \frac{\Lambda}{2}\right)}{\left(\Lambda-4 \tanh \frac{\Lambda}{4}\right)^{2}}
$$

The above reflects an implicit load-deflection solution, with $\Lambda$ determined via the root of equation 3.33. That is, the load-deflection response can be predicted parametrically in terms of the mechanical strain in the beam $\Lambda$ and pre-strain $\bar{\varepsilon}_{R}$ using eqn 3.33 to calculated $\Delta\left(\Lambda, \bar{\varepsilon}_{R}\right)$ and the load $\bar{P}\left(\Lambda, \varepsilon_{R}\right)$ expressed as:

$$
\bar{P}=\frac{\Lambda^{3}}{48\left(\Lambda-4 \tanh \frac{\Lambda}{4}\right)} \Delta
$$

That is, for a given level of mechanical strain in the beam $(\Lambda)$ and pre-strain $\left(\bar{\varepsilon}_{R}\right)$, one can predict the associated deflection using eqn. (3.33) and the corresponding load using eqn. 3.35. Examples of the resulting load-deflection relationships are shown in Figure 3.2 for several values of normalized pre-strain.

An approximate and explicit expression for the load-deflection curve, i.e. $\bar{P}(\Delta)$ can be obtained by recognizing that $F(\Lambda)$ is a weak function of $\Lambda$ across the entire range of possible values, falling from $F(0)=12 / 5$ to $F(\infty)=2$. This is illustrated in Figure 3.3 a; assuming that $F(\Lambda)$ is a constant, $c$, leads to the approximation that $\Lambda=\sqrt{12 c \Delta^{2}+\bar{\varepsilon}_{R}}$. 


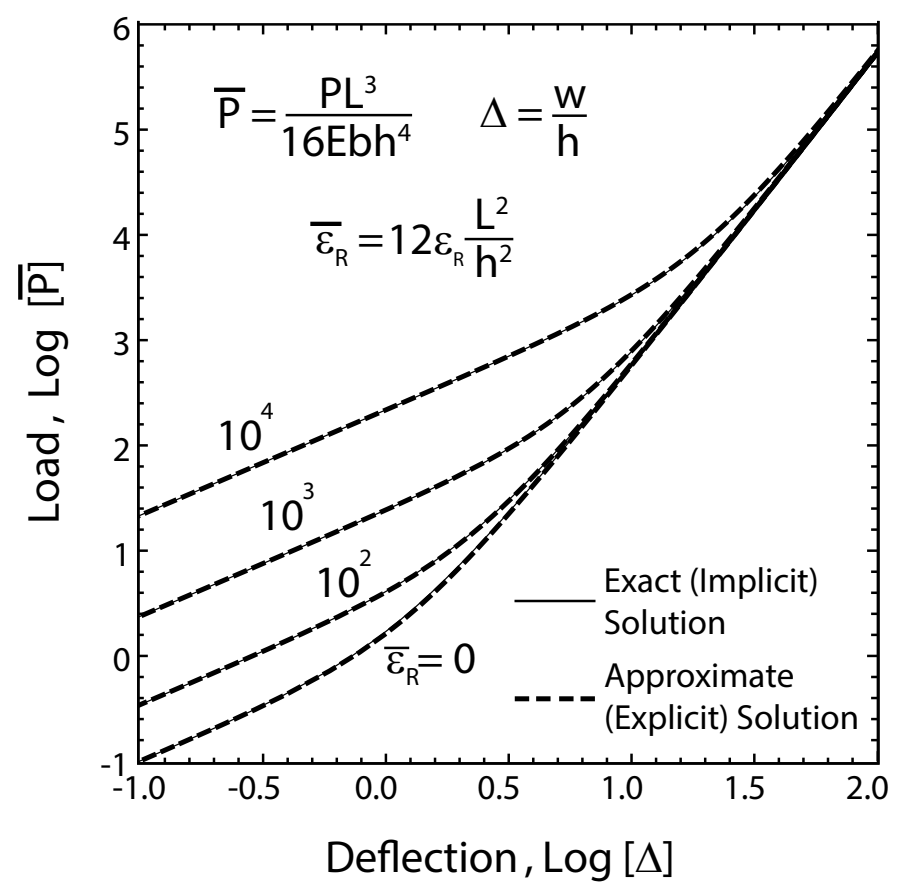

Figure 3.2: Exact and approximate load-deflection relationships for a broad range of prestretch.

Using this approximation in eqn. 3.35, one obtains an explicit relationship for $\bar{P}\left(\Delta, \bar{\varepsilon}_{R}\right)$ :

$$
\bar{P}=\frac{\Delta\left(12 c \Delta^{2}+\bar{\varepsilon}_{R}\right)^{3 / 2}}{48\left(\sqrt{12 c \Delta^{2}+\bar{\varepsilon}_{R}}-4 \tanh \frac{\sqrt{12 c \Delta^{2}+\bar{\varepsilon}_{R}}}{4}\right)}
$$

Adopting $c=2.12$ as an approximation, one obtains a load prediction for a given level of deflection that is within $6 \%$ of the exact solution: a plot of the error in predicted load as a function of applied deflection is shown in Figure $3.3 \mathrm{p}$ for several values of normalized prestrain $\bar{\varepsilon}_{R}$. This expression allows for trivial determination of the modulus and pre-strain from a load-deflection measurement, by least squares fitting of the above non-linear but explicit expression. For completeness, the approximate load-deflection curve is superimposed on the exact solution in Figure 3.2. as expected from the less than 6\% error over the entire range, the differences appear negligible. It should be noted that the exact solution (and hence the approximate form) assumes moderate rotations, which invokes $\sin w^{\prime} \approx w^{\prime}$. This approximation has less than 5\% error for $w^{\prime}<\sim 0.53\left(30^{\circ}\right)$. The consistency check to 

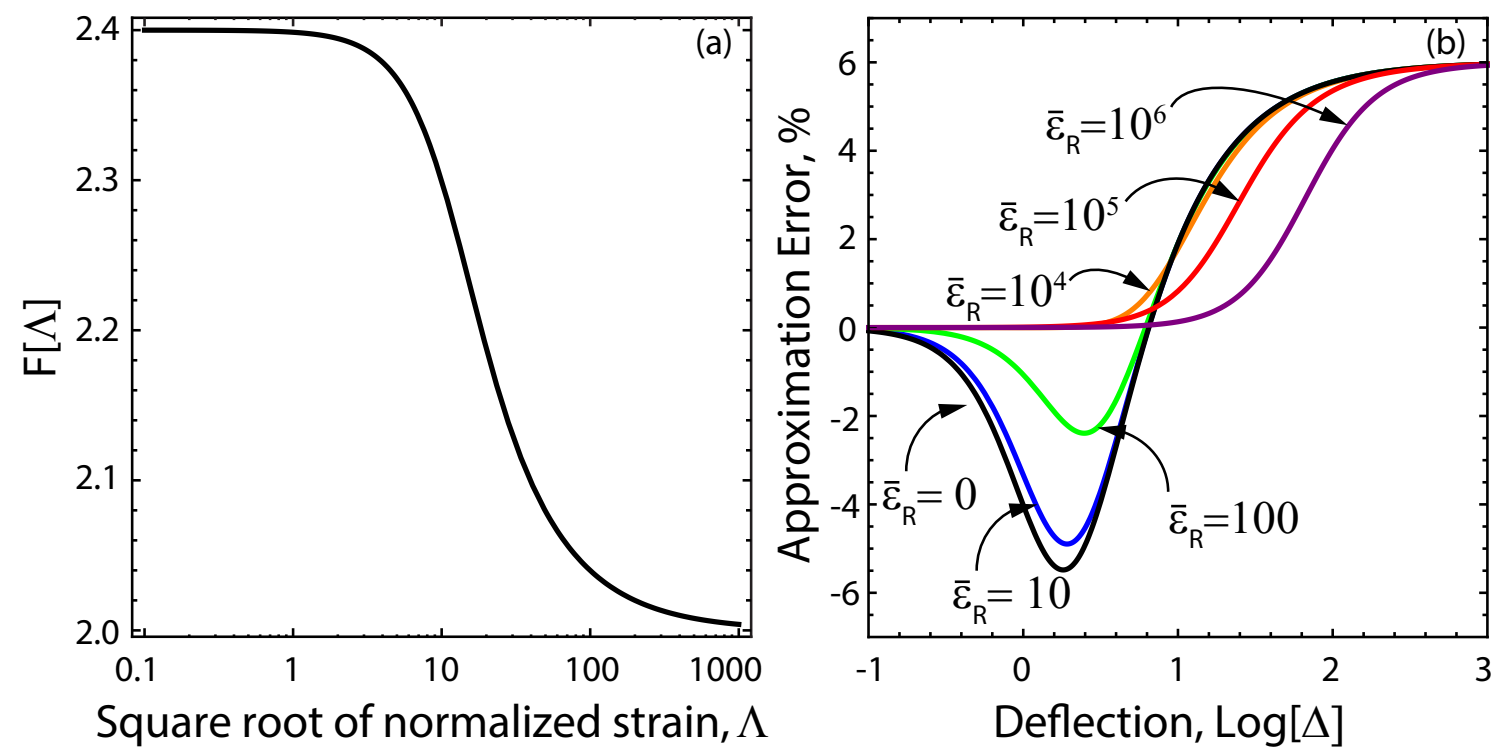

Figure 3.3: (a) The full range of $F[\Lambda]$ and (b) error in the predicted load as a function of applied deflection for values of $\bar{\varepsilon}_{R}$ from $0-10^{6}$ when $c=2.12$.

ensure moderate rotations is therefore given by:

$$
\frac{4 \Delta \sqrt{12 c \Delta^{2}+\bar{\varepsilon}_{R}}\left(\operatorname{sech}\left[\frac{1}{4} \sqrt{12 c \Delta^{2}+\bar{\varepsilon}_{R}}\right]-1\right)}{\sqrt{12 c \Delta^{2}+\bar{\varepsilon}_{R}}-4 \tanh \left[\frac{1}{4} \sqrt{12 c \Delta^{2}+\bar{\varepsilon}_{R}}\right]} \leq \frac{L}{h}
$$

This limit on validity will be reached in most cases when $12 c \Delta^{2}>>\bar{\varepsilon}_{R}$, in which case it is simply $w(L / 2)<L / 4$, i.e. the deflection of the center must be smaller than one fourth the beam length; otherwise, rotations will not be moderate. This simply states that if the deflection shape is a' $\mathrm{V}$ ', the angle of the deformed shape must be less than $30^{\circ}$.

\subsection{Asymptotic limits}

The approximate solution clearly illustrates that linear load-deflection response is observed when $\bar{\varepsilon}_{R}>>12 c \Delta^{2}$, i.e. in the limit of small deflections. The response in this 
scenario is given by:

$$
\bar{P}=\frac{\bar{\varepsilon}_{R}^{3 / 2}}{48\left(\sqrt{\bar{\varepsilon}_{R}}-4 \tanh \frac{\sqrt{\bar{\varepsilon}_{R}}}{4}\right)} \cdot \Delta
$$

This is exact for small deflections, since the choice of $c$ is immaterial upon neglect of the $\Delta^{2}$ term. For small deflections, the additional deformation due to the applied load is negligible in comparison to the pre-strain $\bar{\varepsilon}_{R}$. Critically, it should be noted that this limit captures both bending and membrane stretching: the stiffness of the beam is a function of both the bending stiffness and the pre-strain in the beam.

The role of pre-strain in altering the stiffness of the beam for small deflections can be seen more clearly as follows. The pre-factor to $\Delta$ in 3.38 that depends on $\bar{\varepsilon}_{R}$ represents the increase in stiffness in the linear regime, due to residual stress in the beam. This pre-factor, found by taking a power series expansion of 3.38 about $\bar{\varepsilon}_{R}=0$, is equal to

$$
\bar{P}=\left(1+\bar{\varepsilon}_{R} / 40\right) \Delta .
$$

Similarly the upper limit is taken as

$$
\bar{P}=\left(\frac{\bar{\varepsilon}_{R}}{48}\right) \Delta,
$$

in the limit $\bar{\varepsilon}_{R} \rightarrow \infty$. In the lower limit, bending stiffness still makes a significant contribution to stiffness, while in the upper limit, the stiffness of the beam is entirely due to pre-stretch.

Approximating the pre-factor in 3.38 with $1+\bar{\varepsilon}_{R} / 40$ is within $5 \%$ of 3.38 for $\bar{\varepsilon}_{R} \leq$ 337 , which corresponds to scenarios where stiffening due to pre-stretch is on the same order as the bending stiffness. The upper asymptotic limit (i.e. assuming the pre-factor is $\bar{\varepsilon}_{R} / 48$ ) is within $5 \%$ of the full solution for $\bar{\varepsilon}_{R} \geq 6400$, which simply implies the stiffness increase due to pre-stretch dominates contributions due to bending stiffness. Assuming residual strains on the order of $0.2 \%$, this implies that the stiffness increase of $1+\bar{\varepsilon}_{R} / 40$ 

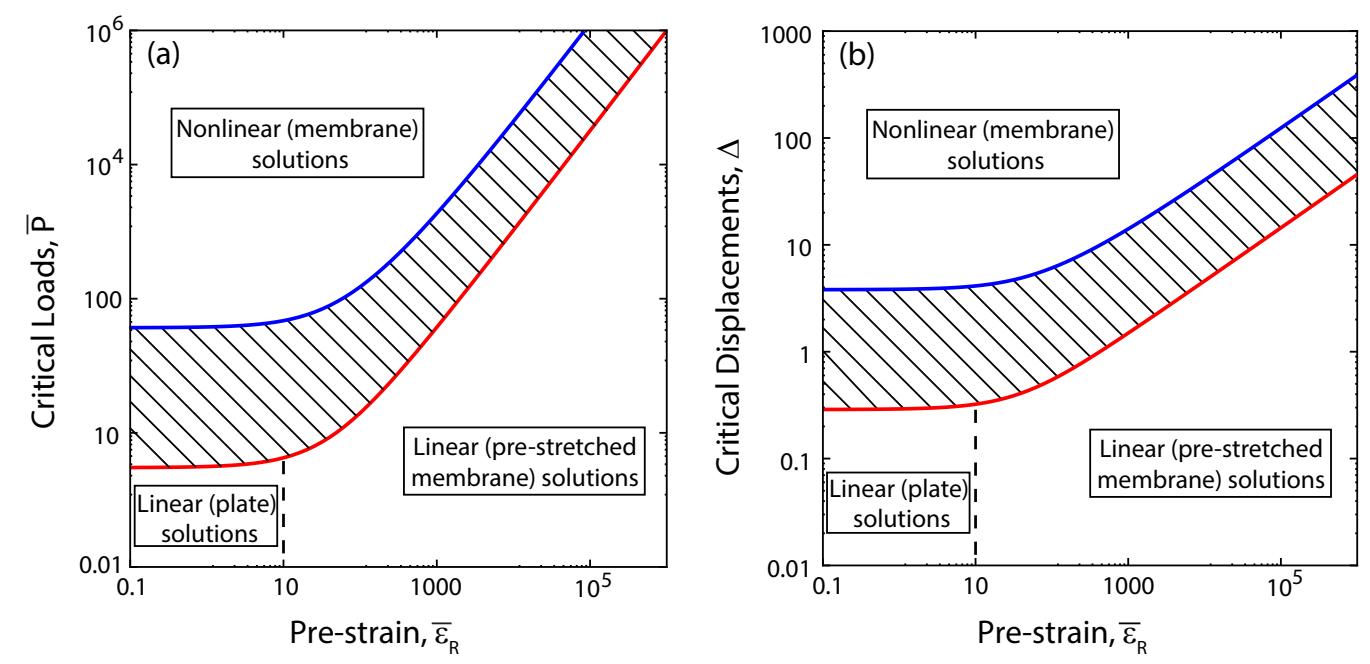

Figure 3.4: Illustration of combinations of (a) normalized critical loads, (b) normalized critical displacements and normalized pre-stretch for which asymptotic solutions are accurate: the shaded region represents the transition from linear regimes to the nonlinear regime where the analytical solution can be used to extract material properties.

is accurate for beams up to $L / h \leq 400$. On the other hand, for $L / h=400$, the upper limit (where stiffness scales with $\bar{\varepsilon}_{R} / 48$ ) is only accurate for residual strains greater than $4 \%$. Considering both asymptotes of the correction with respect to $\bar{\varepsilon}_{R}$, the linear prediction for the stiffness increase (i.e. $1+\bar{\varepsilon}_{R} / 40$ ) is less than $20 \%$ different from eqn. 3.38 for all values of residual strain.

In the other extreme (i.e. large deflections), the approximate solution clearly indicates that classical membrane theory with $\bar{P} \propto \Delta^{3}$ results for scenarios where $12 c \Delta^{2}>>\varepsilon_{R}$. In the asymptotic limit where the deflections are quite large, the strain in the beam scales with $\Delta^{2}$ and the exact solution in this limit corresponds to $c=2$. The approximate solution stated above assumes $c=2.12$, implying a $6 \%$ error in this limit. One can naturally recover an exact expression in the limit of membrane theory using $c=2$. The error in the approximate form for intermediate deflections (see Figure $3.3 \mathrm{~b}$ ) will be larger than $6 \%$, and hence, $c=$ 2.12 is used in the following: it produces relatively small errors for all scenarios. Again, it should be noted that for large deflections, one must check the size of the rotations (i.e. $\left.w^{\prime}(x)\right)$ to ensure the moderate rotation approximation is valid. 


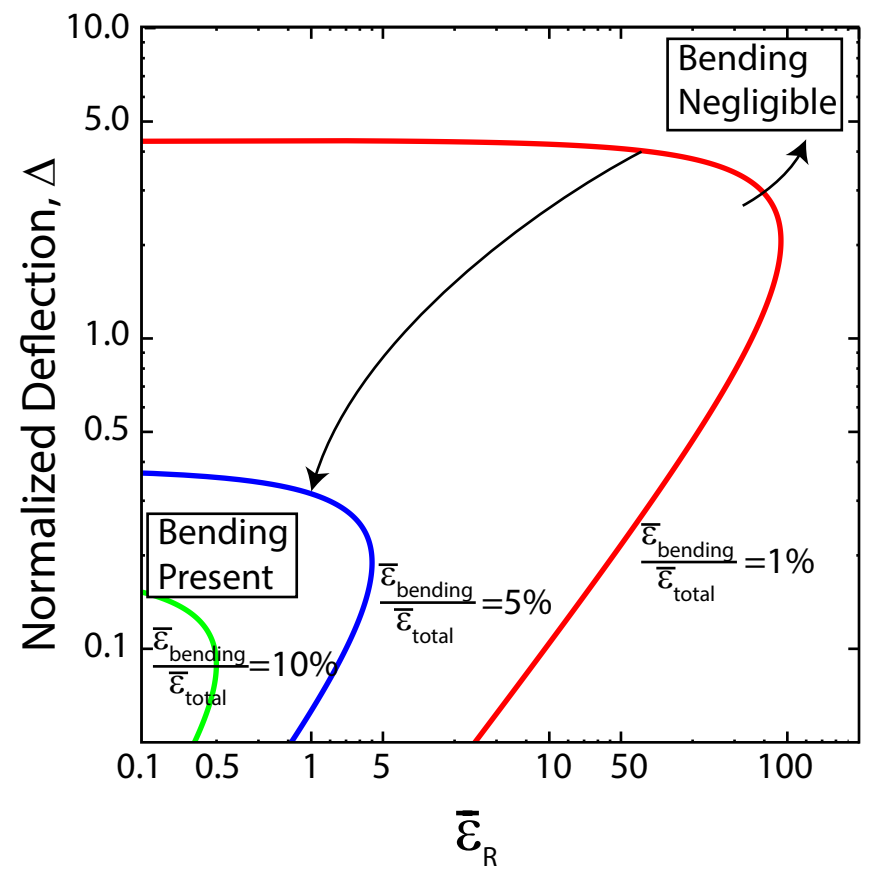

Figure 3.5: Contours showing combinations of pre-strain and deflections where the contribution of bending strain to the total strain in the beam is 1, 5 and 10\%. For applied displacements greater than $\sim 5$ times the film thickness bending strains are negligible regardless the level of pre-strain.

\subsection{Design of experiments}

Given the sophistication of modern instrumentation, tests often span multiple response regimes. To guide the design of experiments that corresponding to a specific regime, Fig. 3.4 shows combinations of loads, displacements and pre-stretch where solutions are governed by linear, membrane and the transition from linear to non-linear membrane behavior. The transition region, denoted by hatching in the Fig. 3.4, is represented by eqn. 3.36 . Upon assuming properties of the material being tested, it is possible to choose beam geometries that will position a large portion of the test in a particular regime. For example if neither modulus or pre-strain are known, it is desirable to test the beam in the mixed region, because non-linear coupling allows for accurate extraction of both properties. This technique will be shown in the experimental study discussed below.

In addition to identifying the relevant type of response in a given scenario, the above analysis also provides insight into values of pre-strain and applied deflection where stretch- 
ing strains dominate bending strains. In some instances, notably the design of tests to explore non-linear material response, it is desirable to by-pass small deflection behavior in favor of the regime associated with pure stretching. While we do not consider non-linear material response here, the use of the present model is nonetheless informative, as we seek to eliminate the bending regime, which generally corresponds to small strains where elasticity plays a role. The maximum bending strain occurs at the outer skin, i.e. $y=h / 2$ : one can calculate the bending strain at this location for a given position according to:

$$
\bar{\varepsilon}_{\text {bending }}(\bar{x})=\frac{L^{2} \varepsilon_{\max }(\bar{x})}{12 h^{2}}=\frac{1}{48} \bar{w}^{\prime \prime}(\bar{x})
$$

As one would expect, the bending strains are maximum at the clamp and under the pointload (and those two are equal, because of symmetry).

Using the approximation that $\Lambda \approx \sqrt{12 c \Delta^{2}+\bar{\varepsilon}_{R}}$, one obtains the following for the maximum bending strain in the beam:

$$
\bar{\varepsilon}_{\text {max }}=\frac{\Delta \sqrt{12 c \Delta^{2}+\bar{\varepsilon}_{R}}}{24\left(\sqrt{12 c \Delta^{2}+\bar{\varepsilon}_{R}} \operatorname{coth} \frac{\sqrt{12 c \Delta^{2}+\bar{\varepsilon}_{R}}}{4}\right)-96}
$$

The above can be used to estimate the deflection that is required to reach yielding at the clamps. The ratio between the contribution due to bending, the last term, and the total strain is of central interest: for large enough deflection, bending strains are negligible and membrane approximations are valid.

$$
\frac{\bar{\varepsilon}_{\text {bending }}}{\bar{\varepsilon}_{\text {total }}}=\frac{\Delta}{\Delta+24\left(\sqrt{12 c \Delta^{2}+\bar{\varepsilon}_{R}} \operatorname{coth} \frac{\sqrt{12 c \Delta^{2}+\bar{\varepsilon}_{R}}}{4}\right)-96}
$$

Though not immediately obvious, the strain ratio given above asymptotes to unity when $\Delta \rightarrow 0$, as expected in the small-deflection, pure bending limit. Figure 3.5 illustrates critical values of residual strain and applied displacement where bending strains contribute a given percentage of the total strain, again assuming $c=2.12$. The results illustrate that 
pure stretching is obtained for nearly all values of applied displacements (even small ones) when the residual strain is large, as one expects. It is interesting to note that for all values of residual strain bending contributes less than $1 \%$ of the total strain for $\Delta>5$. This is probably an upper limit for the required displacement, because yielding at the clamps and load point causes plastic hinges to form, such that bending is further reduced.

\subsection{Geometric formulation}

In the case where the beam is a membrane loaded in tension it is useful to examine the loading of the beam from a purely geometric approach in order to recreate a traditional stress strain curve commonly used to extract material properties such as residual stress, yield stress, fracture stress and fracture strain. Figure 3.5 clearly shows that at moderately large values of deflection relative to film thickness $(\Delta>5)$, bending effects are negligible and can be ignored in the membrane formulation. Additionally it is assumed that the load is distributed evenly across the cross-section of the beam as the beam is pushed further into the membrane stretching regime. Figure 3.6 shows the relevant loads and displacements necessary to derive stress in the cross-section of the deformed beam. It should be noted that strain has been derived elsewhere as eqn. 2.18 and is simply:

$$
\varepsilon=\sqrt{4\left(\frac{\delta}{L}\right)^{2}+1}-1
$$

The angle between the undeformed and deformed beam states, $\theta$, is defined as:

$$
\theta=\tan ^{-1}\left(\frac{2 \delta}{L}\right)
$$

Realizing the that load in the cross-section, referred to here as the membrane load $P_{m}$, can be defined as a function of the applied load, $\mathrm{P}$, and $\theta$ :

$$
P_{m}=\frac{P}{2 \sin (\theta)} \text {. }
$$




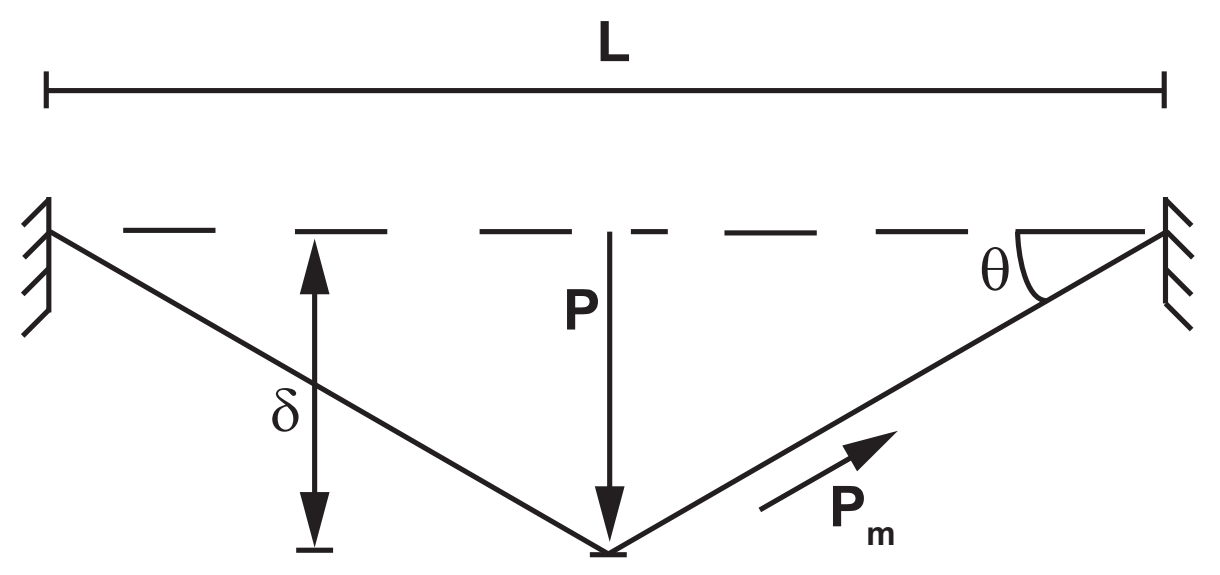

Figure 3.6: Relevant loads, displacements and angles used to geometrically derive stress and strain

The load in the cross-section divided by the cross-sectional area yields the stress in the cross section:

$$
\sigma=\frac{P}{2 A \sin (\theta)}
$$

It should be noted that this formulation assumes a loading function where the load is evenly distributed through the cross-section of the beam. It is evident that the effects of residual stress in the beam are not included in this analysis. When stress is plotted as a function of strain, residual stress appears as the intercept on the stress axis. Discussion of this result with respect to experimental testing will be addressed in Chapter 6. 


\section{Chapter 4}

\section{Material Properties of npAu and AuAg}

\section{Films and Beams}

Nanoporous gold (npAu) has been shown to have promising applications in sensors and coatings due to its increased surface area, chemical inertness and biological compatibility [39. [40]. Using freestanding structures to characterize nanoporous gold yields insight into mechanical properties of these films and allows comparisons to be made regarding the links between microstructure and properties. Of additional interest is how the evolution of npAu changes depending on the geometric constraints (i.e. film-on-substrate (FOS), fixed-fixed beam or cantilever), especially with regard to films that have been subjected to thermal loading. In conjunction with microstructural studies and wafer curvature stress measurements [1, 2, 41], the effects of pre and post fabrication annealing on the mechanical properties and fabrication yield of npAu beams, cantilevers and FOS were investigated via nanoindentation. Preannealing here refers to thermally treating AuAg beams after they are released but before they are dealloyed which is shown to be a necessary step in order to maximize fabrication yield[1]. Postannealing refers to thermally treating beams postrelease and after dealloying to alter the microstructure and properties of beams. A detailed description of geometric and porosity characterization can be found elsewhere[2, 41] 1 . The

\footnotetext{
${ }^{1}$ It should be noted that all geometric characterization and residual stress on blanket film measurements were performed by Erkin Şeker. All figures and work that use parts of his work are cited appropriately.
} 


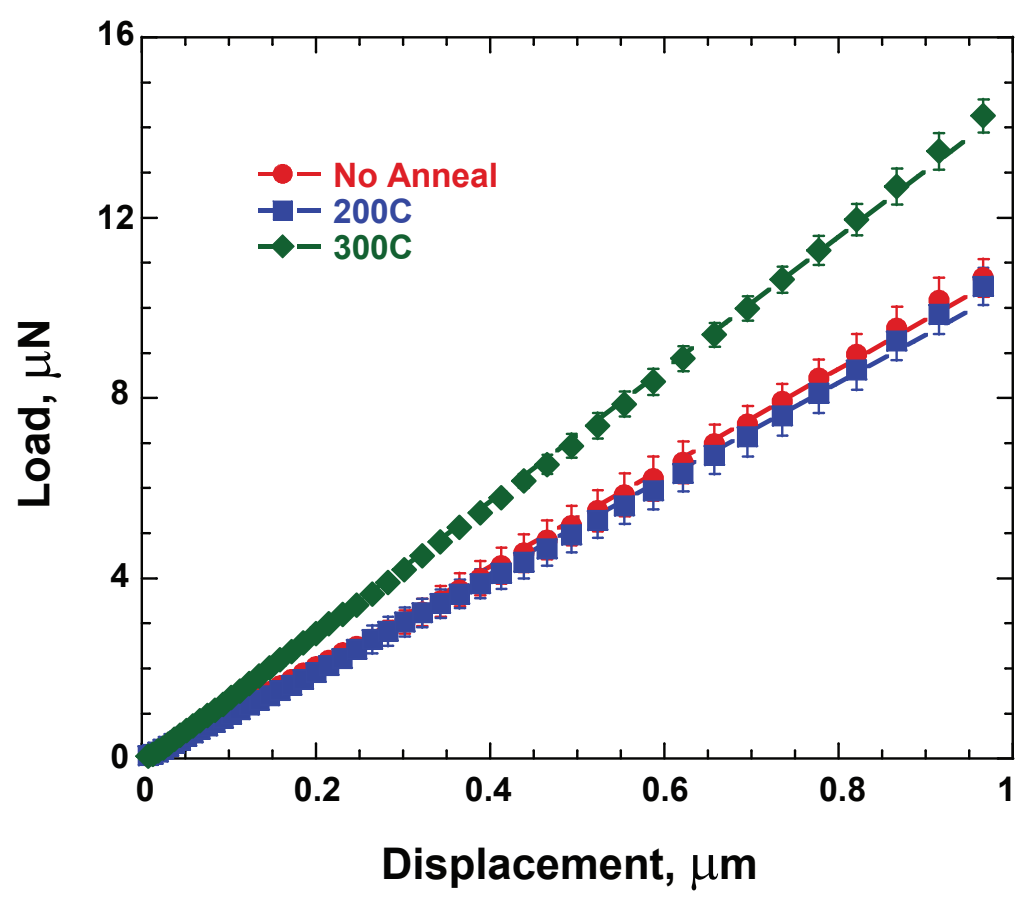

Figure 4.1: Representative load-displacement curves on beams on $100 \mu \mathrm{m}$-long clamped npAu beams with various heat treatments

focus here will be to show the use of nanoindentation to extract material properties from films and structures that are particularly challenging due to their low stiffness. These tests serve as experimental validation of the testing method set out in Chapter 2 and a portion of the test interpretation from Chapter 3.

\subsection{Fixed-fixed beams}

Beams were probed using the DCM head with a Berkovich diamond tip using the surface find method described in Chapter 2 with an oscillation height of 15nm for AuAg beams and $20 \mathrm{~nm}$ for $\mathrm{npAu}$ beams. Three different beams from beam sets with length 100 and 150 $\mu \mathrm{m}$ were tested for each annealing temperature. Each beam was indented at its center three times to ensure test repeatability and confirm that there was no anelastic deformation occurring at small scale deformations which are assumed to be entirely elastic for the analyses to follow. Representative load-displacement curves are shown in figure 4.1. Error bars are 
the standard deviation of nine measurements for each annealing temperature. Only loading curves are shown; unloading curves are virtually identical but have been removed to simplify the figure. The results clearly demonstrate that the fabrication and measurement approaches produce highly repeatable results. A mixture of 15 and $30 \mu \mathrm{m}$ wide beams for each length was tested, and it was observed that there was not a significant difference in the stiffness of the two widths when scaled by the difference in width.
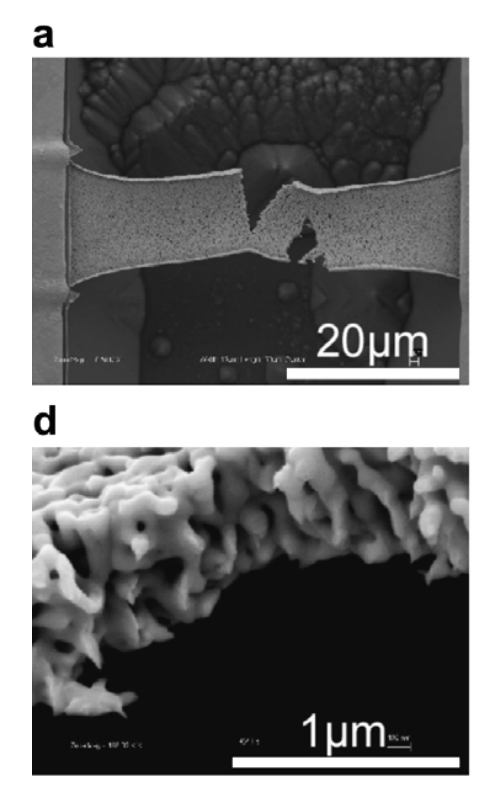

b

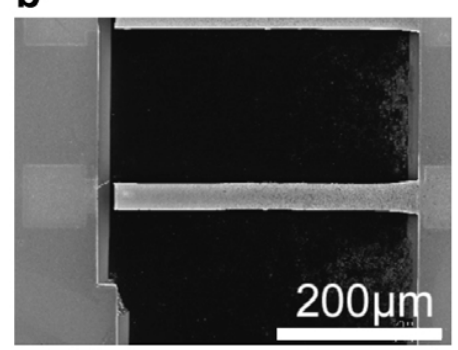

e

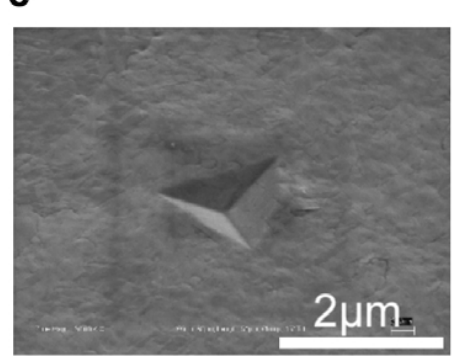

C

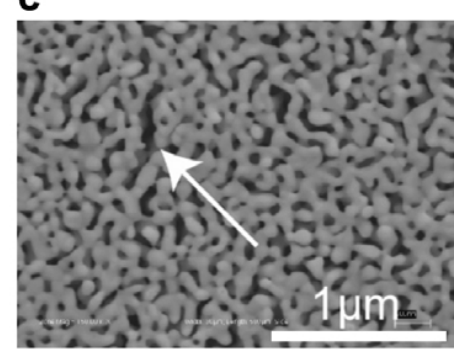

f

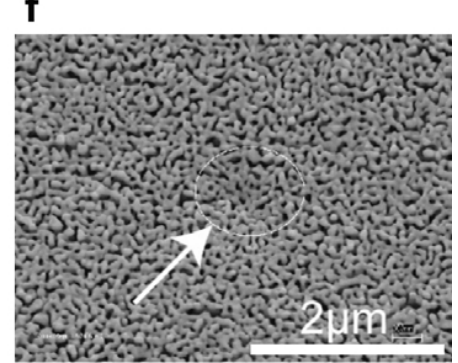

Figure 4.2: npAu beam which has fractured during $400^{\circ} \mathrm{C}$ heat-treatment (a). Tensile beam failure of beam-clamp interface caused by skipping heat-treatment prior to dealloying discussed elsewhere[1] (b). Micro-crack formation during heat-treatment at $200^{\circ} \mathrm{C}$ (c). Micronecking where a npAu beam has been broken with tweezers(d). Nanoindenter tip mark on electroplated Au clamp (e), and npAu beam surface (f).[2]

Indenter penetration into the film, as opposed to beam deflection, is also negligible as seen in figure 4.2 f. Contact penetration into the beam leads to non-linear behavior because of the growing contact area during penetration as shown earlier in eqn 2.3 . The relatively blunt Berkovich indenter tip quickly generates a contact stiffness exceeding that of the beam; hence, the beam deflection dominates the response for probe displacements greater than $\sim 20 \mathrm{~nm}$, as seen in figure 4.1. The results in figure 4.1 are used to calculate the residual stress in clamped beams. For very small loads, the initial load-deflection response is linear, 


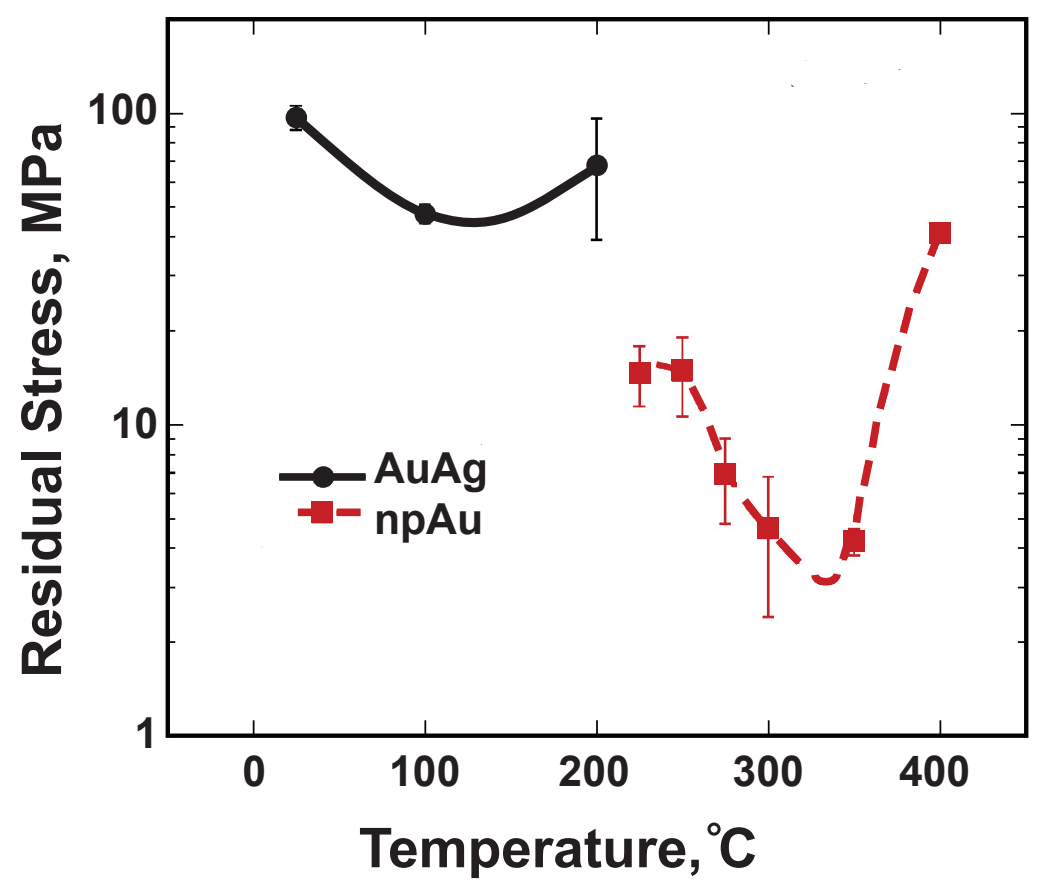

Figure 4.3: Residual stress as a function of pre-annealing for $\mathrm{AuAg}$ beams and npAu beams. The residual stress in the npAu beams is post dealloying, prior to additional annealing.

regardless of whether bending or residual stress dominates. Remembering from Chapter 3 that the small displacement, small prestrain limit is:

$$
\bar{P}=\left(1+\bar{\varepsilon}_{R} / 40\right) \Delta
$$

the residual stress in the beams can be calculated and is shown in figure $4.6 \mathrm{p}$. All beams have $\bar{\varepsilon}_{r} \leq 337$, which is the cutoff for an approximation error of 5\%. Again the error bars are due to the standard deviation from nine distinct load-displacement measurements per annealing temperature.

AuAg beams were also tested and eqn. 4.1 was used to extract residual stress. Figure 4.3 shows the results of residual stress testing on AuAg and npAu beams where the temperature associated with the npAu beams is the preannealing temperature on the $\mathrm{AuAg}$ beams prior to dealloying. It was found that preannealing at $300^{\circ} \mathrm{C}$ induced plastic buckling 


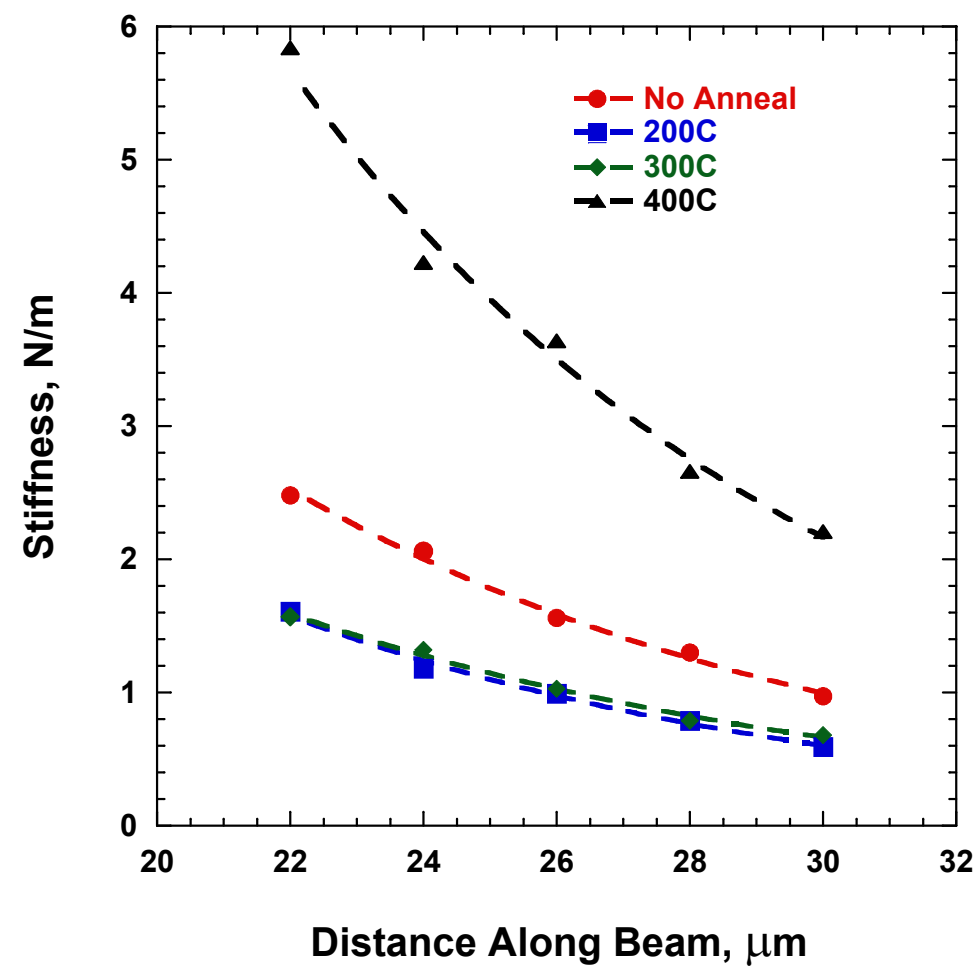

Figure 4.4: Stiffness of npAu FIB-cut cantilevers as a function of distance from the clamp which allow for independent determination of elastic modulus; curves are least-squares fit of the form $\mathrm{k}=\mathrm{c} / \mathrm{l}^{3}$

and counteracts the tensile residual stress from the dealloying process, leaving the beams flat and relatively unstressed. It is not surprising this preannealing temperature also produces the highest yield. The residual stress for beams in figure 4.6 is from beams that have been pre-annealed at $300^{\circ} \mathrm{C}$ and then annealed again. The tensile residual stress increased monotonically with increasing annealing temperature for np-Au beams and is attributed to densification which translates into increasing tensile stress with heat-treatment.

\subsection{FIB-cut cantilevers}

The microstructural evolution of clamped beams and cantilevers respond differently to annealing temperature. As such, "broken" cantilevers made from fractured beams similar to those seen in figures $4.2 \mathrm{a}$ and $\mathrm{b}$ could not be used to determine the elastic modulus of 
annealed beams. Accordingly three annealed beams from 15 and $30 \mu \mathrm{m}$ wide beams were cut into cantilevers using a FIB. Elastic modulus measurements were obtained based on load-deflection measurements. FIB-cut cantilevers were indented at successive distances down the beam (i.e. $22-30 \mu \mathrm{m}$ in $2 \mu \mathrm{m}$ steps) from the edge of the clamp. Three beams were tested for each annealing condition. This allowed an independent calculation of average elastic modulus for each annealing condition. Stiffness measurements as a function of distance from the clamped edge are shown in figure 4.4 which are used to infer the modulus of the beams using the Euler-Bernoulli beam theory:

$$
k=\frac{E b h^{3}}{4 l^{3}},
$$

where $\mathrm{k}$ is the measured cantilever stiffness, $\mathrm{b}$ is the beam width, $\mathrm{h}$ is the beam thickness and 1 is the distance from the clamp to the contact point. Figure 4.4 clearly shows the predicted relationship between stiffness and probe location, again indicating the lack of any significant plastic deformation or indenter penetration. The elastic modulus calculated from the stiffness measurements is shown in fig. 4.6. It is important to note that this modulus corresponds to that of the clamped beams, since cantilevers were created by cutting intact clamped beams post-anneal. These modulus values were the ones used in the calculation of residual stress from the point-loaded beam tests in the section 4.1. There is a slight initial decrease in elastic modulus with increasing annealing temperature suggesting there are two competing mechanisms altering the stiffness of clamped structures. Higher temperatures encourages densification, lowering porosity and increasing elastic modulus similar to that seen for the $400^{\circ} \mathrm{C}$ annealed case. For lower annealing temperatures densification is occurring but the increased tensile stress cracks ligaments, lowering the effective modulus. An example of this ligament failure can be seen in fig. $4.2 \mathrm{k}$ and in the fractured case in fig. $4.2 \mathrm{~d}$ where necking of ligaments can be seen. The test on these cantilevers and the beams from the section above are many of the experimental points used in figure 4.5 to verify the validity of the model used to predict the coupled response of the beam/indenter system. 


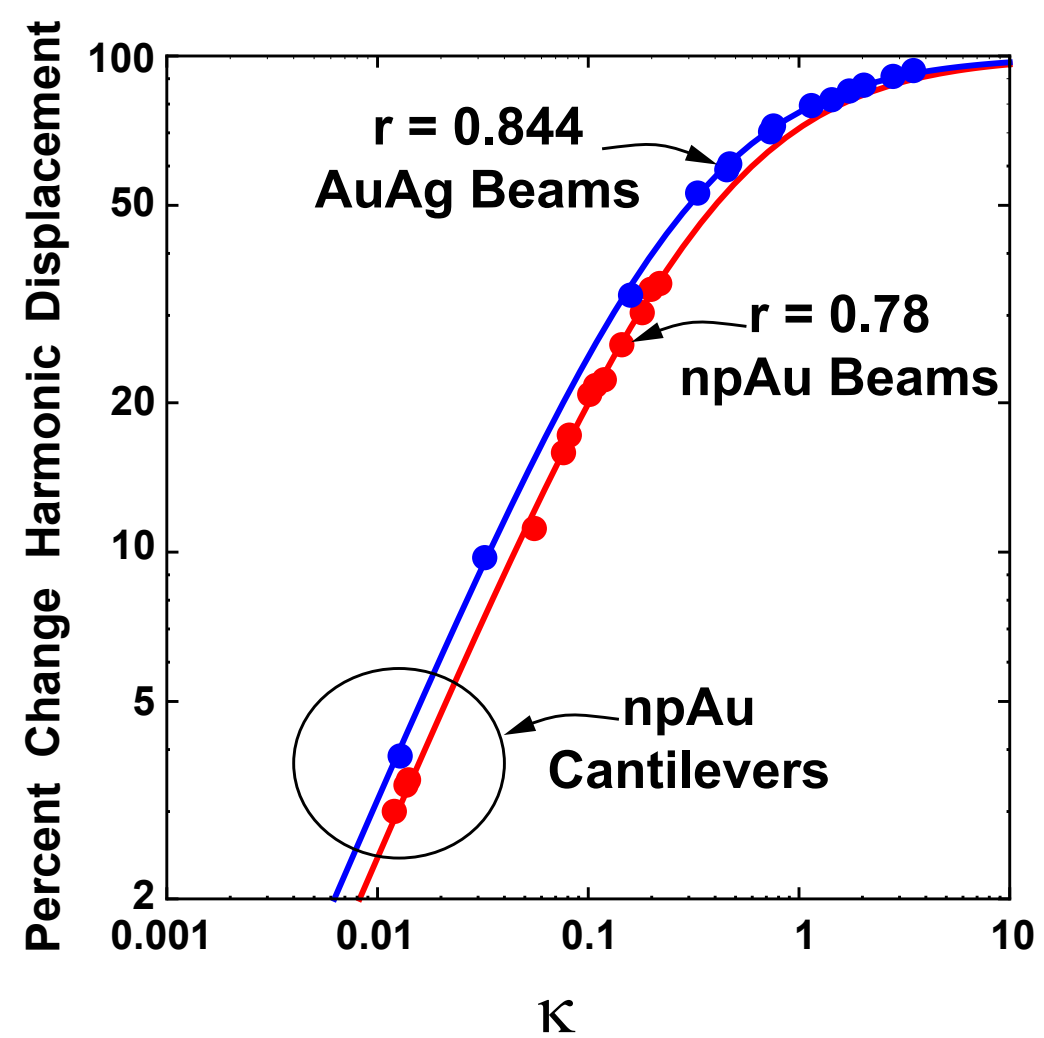

Figure 4.5: Percent change of harmonic displacement as a function of beam stiffness and operating frequency for tests on $\mathrm{AuAg}$ and npAu beams. Lines running through data are theory from chapter 2 .

\subsection{Film-on-substrate}

Blanket films were characterized via conventional nanoindentation techniques, using the DCM head with a Berkovich tip. The tip geometry was calibrated on a quartz sample to determine the tip area coefficients so that modulus could be determined as a function of indentation depth on the blanket films. The calibration in figure 4.7 is valid (i.e. modulus id independent of indentation depth) for depths greater than $\sim 20 \mathrm{~nm}$. The results on $\mathrm{npAu}$ indicated that the elastic modulus was independent of penetration depth for depths less than $\sim 100 \mathrm{~nm}$. For depths greater than $\sim 100 \mathrm{~nm}$ an increase in modulus is seen due to effects of the substrate. The reported modulus values are averages from 10 indents taken over 

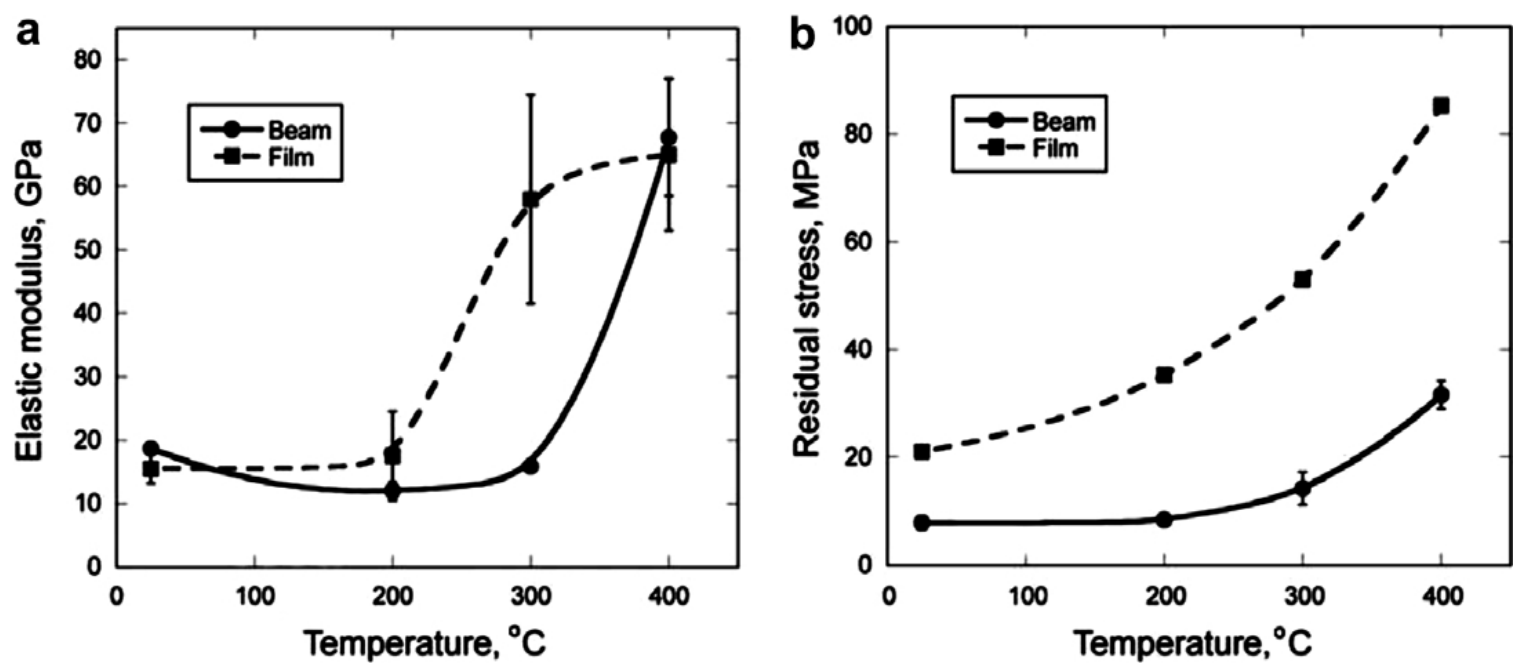

Figure 4.6: Elastic modulus calculated from tests on npAu FIB-cut cantilevers and blanket FOS samples(a). Residual stress calculated from center span point loaded tests on fixedfixed npAu beams and wafer curvature measurements on continuous films(b).[2]

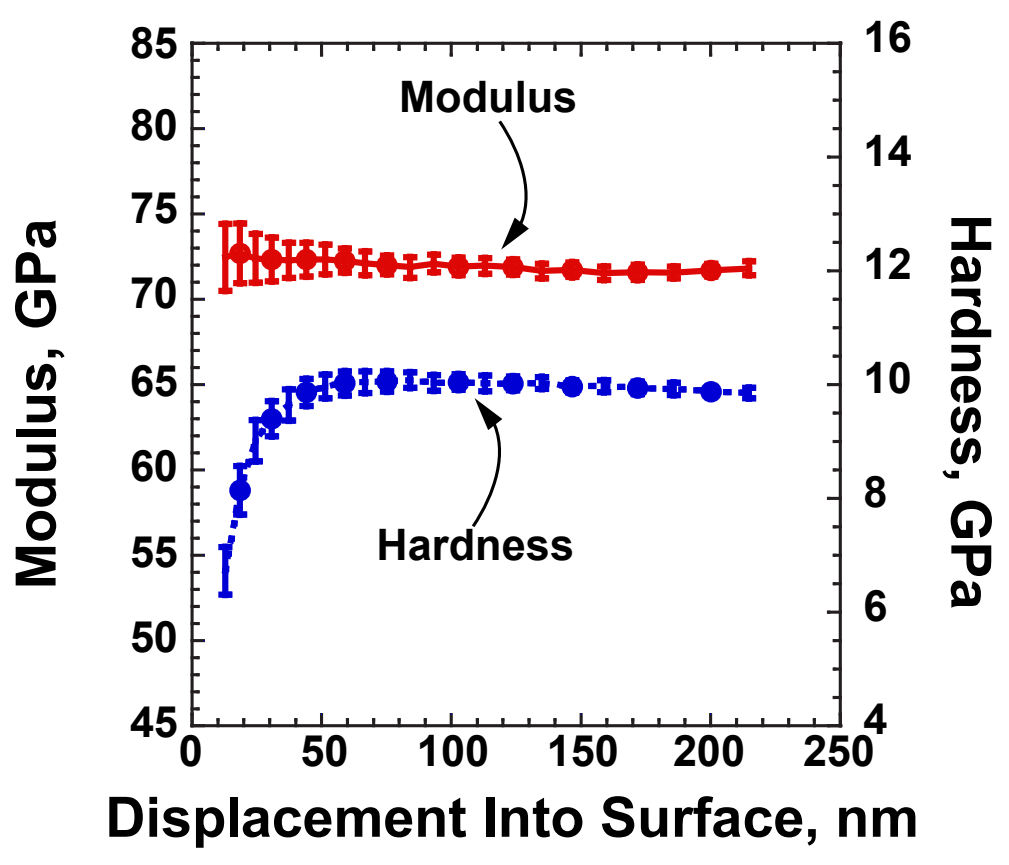

Figure 4.7: Residual stress as a function of pre-annealing for AuAg beams and as a function of post-annealing for npAu beams. Residual stress in the AuAg beams appears to reduce as a function of annealing temperature while the stress state in the npAu beams is variable depending on competing microstructural mechanisms. 
depths ranging from 20 to $100 \mathrm{~nm}$. Modulus was extracted from FOS testing of blanket films and was found to be $72 \pm 2 \mathrm{GPa}$ for all annealing conditions. The averaged results in figure $4.6 \mathrm{a}$ are from tests on blanket npAu films and cantilevers made by using a FIB to cut fixed-fixed beams. Residual stress measurements presented in fig. 4.6p for the FOS sample were measured via wafer curvature measurements described elsewhere and were performed by Erkin Seker[2]. As is expected, the elastic modulus increases with annealing temperature as the films densify reaching a maximum value that is around ten percent of the bulk gold value. Further information regarding the links between microstructural changes and mechanical properties of these films may be found elsewhere[1, 2, 41]. 


\section{Chapter 5}

\section{Fabrication and Film Characterization}

\section{of Nickel Beams}

\subsection{Fabrication}

There are a host of methods used to make test specimens for microscale mechanical testing. Many of these processes involve complicated lithography steps, high thermal treatments and exposure to harsh chemical etchants. A key advantage of the fabrication process described below is simplicity. There are two masks involved in fabrication, beams can be fabricated directly on silicon, the etch is dry and uncomplicated and there is a minimal thermal budget involved including zero thermal treatment post release. The following sections describe the details, difficulties and solutions to issues associated with fabrication. The tools and methods used to characterize film geometries and microstructures will also be discussed.

\subsubsection{Beam design}

A goal of this work was to be able to test beams to a large strain level such that fracture was achieved prior to reaching the maximum travel of the indenter. This dissertation focuses on shorter thinner films, however, the following mask design includes longer beams 


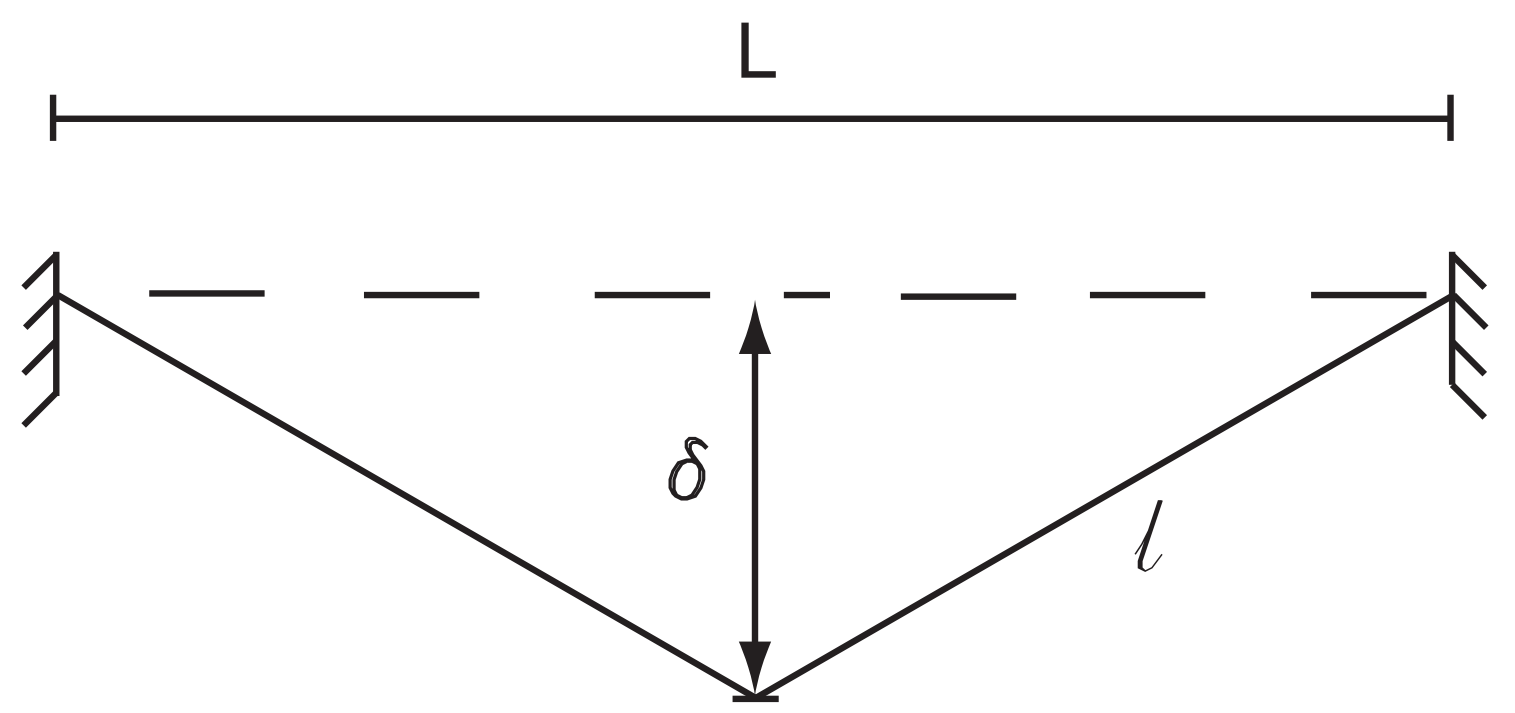

Figure 5.1: Representative diagram of stretch dominated MDE test.

which would allow for thicker (i.e. electroplated) films to be tested. Figure 5.1 again shows the representative geometry of an MDE test. Remembering the strain in the beam from eqn. 2.18 as

$$
\varepsilon=\sqrt{4\left(\frac{\delta}{L}\right)^{2}+1}-1 .
$$

allows an examination of the limits of the indentation system with regard to beam design.

The DCM (low-load) indentation head is limited to $30 \mu \mathrm{m}$ of displacement. For a beam with a length of $200 \mu \mathrm{m}$, this is a corresponding strain of $\sim 4.5 \%$ at $30 \mu \mathrm{m}$ of displacement. For most applications in thin films, especially those with nanocrystalline grain distributions, fracture will precede this as seen in some recent studies[12, 19, 42]. Accordingly we chose lengths of 200, 300, $500 \mu \mathrm{m}$ and $1 \mathrm{~mm}$ combined with widths of 20,30 and $50 \mu \mathrm{m}$ to create twelve different length/width combinations each with five beams per combination. Additionally, a few combinations of cantilevers have been included on the mask. The longer beams will achieve lower strain levels at $30 \mu \mathrm{m}$ of displacement but allow for thicker films to be tested with a high load head which also has a higher displacement range. For 


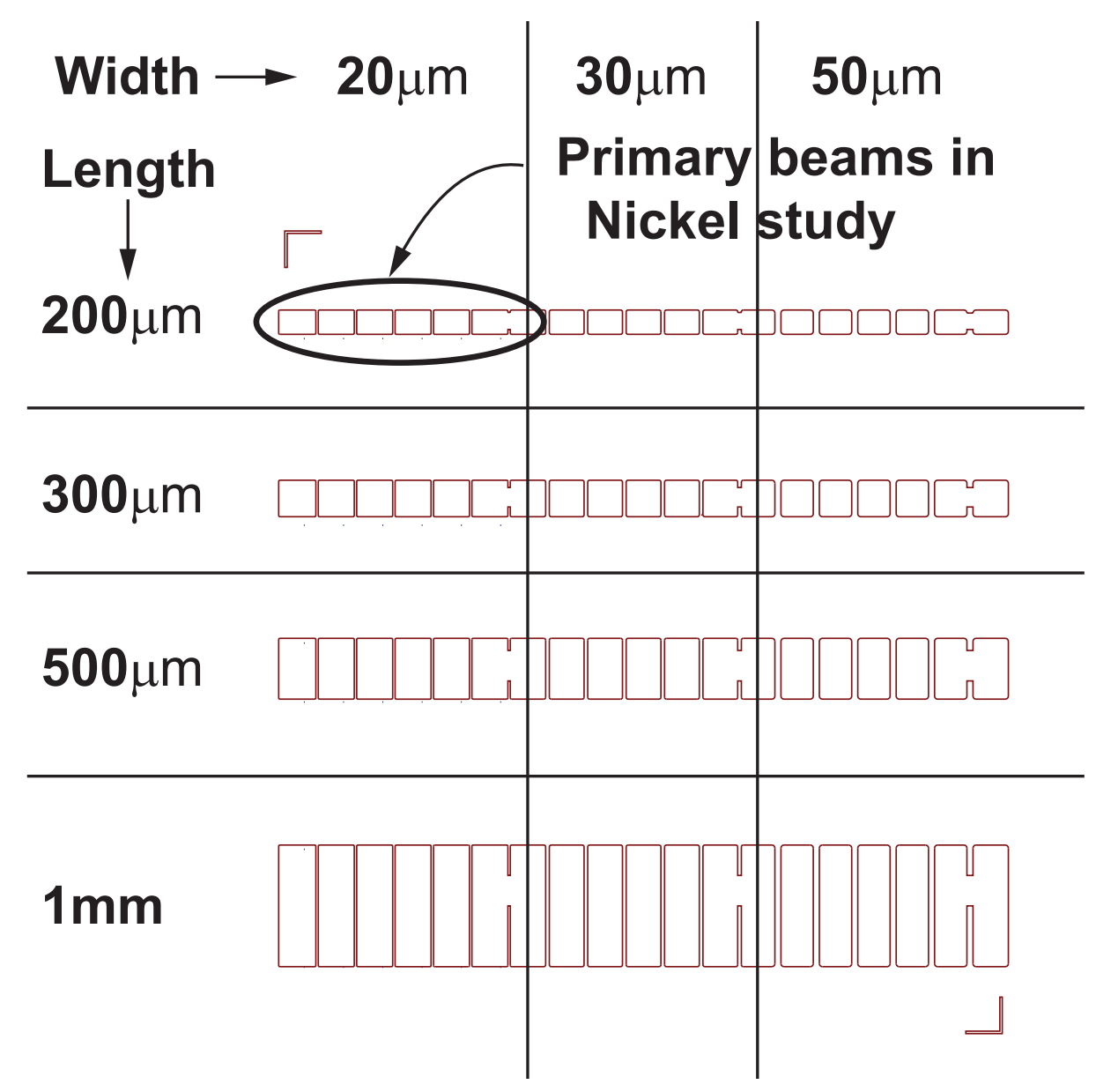

Figure 5.2: Mask 3 used to define beam geometry. Positive resist profile defines evaporation, sputter and electroplating depositions. Negative resist profile can be used to etch beams from a continuous film.

results presented in later chapters, all beams are of nominal length and width $200 \mu \mathrm{m}$ and $20 \mu \mathrm{m}$ respectively in order to ensure fracture prior to reaching the maximum displacement limits.

The beam mask in Figure 5.2 was used for fabricating beams. In order to ensure there were no stress concentrations at the ends of beams, which would act as initiation sites for failure during testing, the corners at the film-beam interface were filleted with a radius equal to the beam width as seen in Figure 5.3. All mask designs were created using AutoCAD and were produced by Microtronics Inc. [43] Appendix 8.1 has the complete mask layout. 


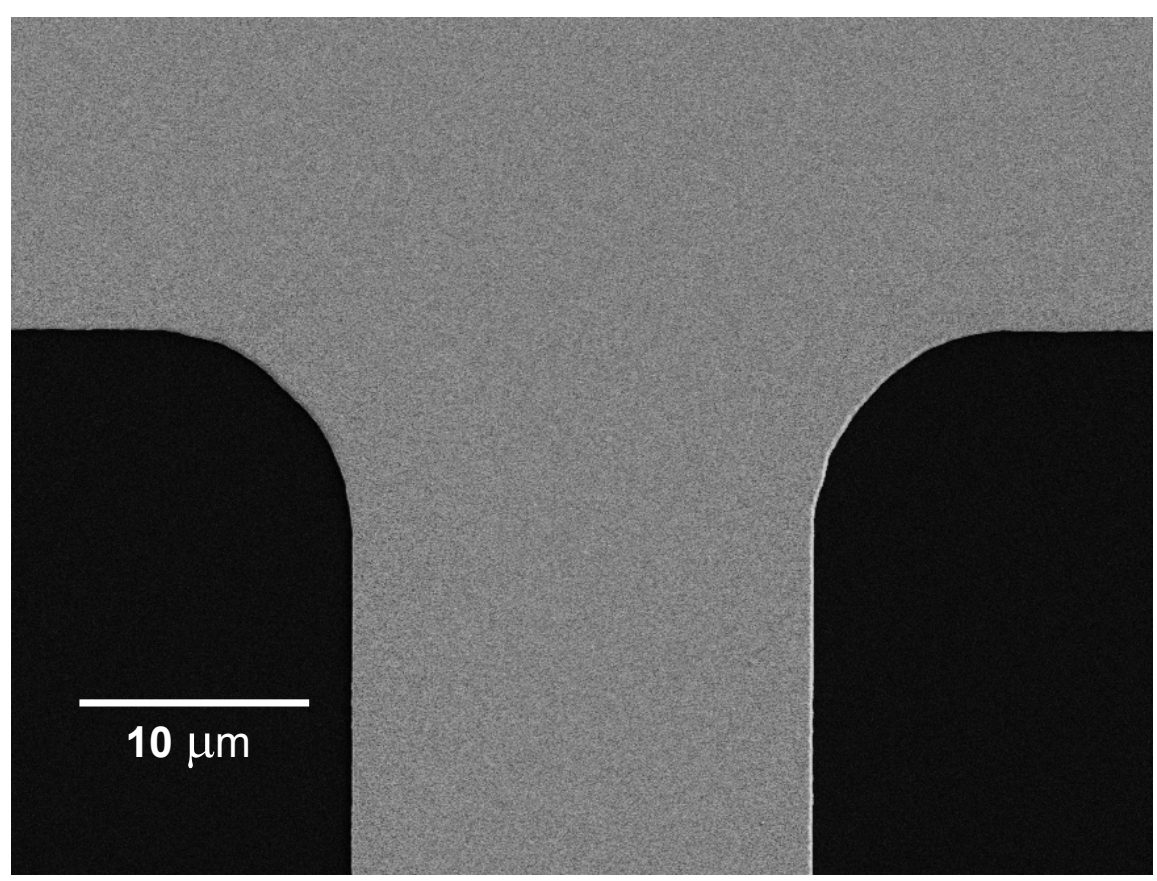

Figure 5.3: SEM image of filleted corner to reduce stress concentrations.

\subsubsection{Beam Fabrication}

This section describes the fabrication process to create freestanding Nickel beams. For this process silicon must be the substrate as it is not only the substrate but also the sacrificial layer. Full process sheets can be found in Appendix 8.2. All fabrication procedures described use etch mask 3 from Figure 5.2 to define beam geometries.

Beams were fabricated on $325 \mu \mathrm{m}$ thick 2-inch $p$-type 100-oriented silicon which acts as the substrate as well as the sacrificial layer. The wafers were initially cleaned with ethanol, trichloroethylene (TCE) and methanol, or ETM clean, before any fabrication steps were carried out. Prior to depositing the beam film, a bilayer photoresist film (lift-off resist LOR10B and positive photoresist AZ4210) was spun on and patterned, which produces a controlled degree of undercut on the bottom layer, preventing irregularities at the bridge edges after the lift-off process. Figures 5.4 and 5.5 show the representative lift-off stack used in this fabrication procedure. A titanium adhesion layer, of nominal thickness 10nm, was first deposited followed by the nickel beam layer. The Nickel (99.995\% pure) beams 
a)

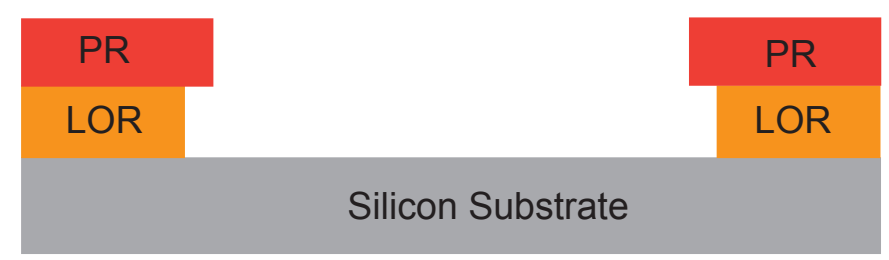

b)

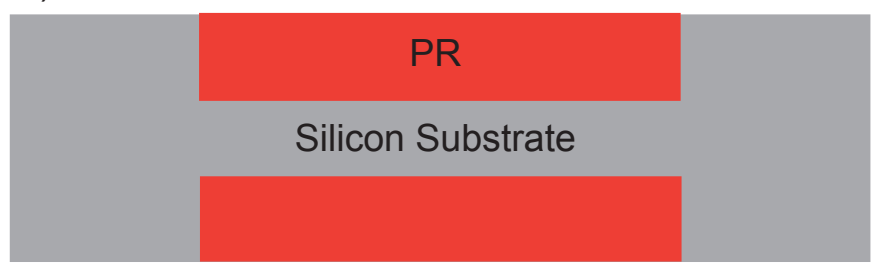

Figure 5.4: Representation of lift-off stack for beam fabrication.

were deposited via electron beam evaporation with a base pressure of $9 \times 10^{-7}$ Torr. Deposition rates were one and two angstroms per second for titanium and nickel, respectively. In order to minimize thermal effects on the beams after deposition, lift-off of the excess metal and PR was performed in room temperature $n$-methyl-2-pyrrolidone(NMP) with the help of a low power ultrasonic bath. Following lift-off the films were cleaned using the ETM clean.

\subsubsection{Alternative Fabrication Methods}

It is important to note beams can be fabricated using a number of similar methods to yield similar beams. Three additional methods to fabricate beams are sputter deposition, electroplating and etching of a continuous film. Fabrication with sputter deposition is achieved by the same steps outlined above, replacing the electron beam evaporation step with sputter deposition. It should be noted that sputter deposition often involves film deposition at an angle which increases the importance of the controlled undercut seen in figure 5.4 $\mathrm{a}$ in order to avoid "flagging" on the beam edges where material is deposited on the PR side wall. Etching a continuous film can be accomplished by reversing the pattern in figure 5.2 by either producing another mask or by using negative PR which requires spinning resist onto the metal surface and applying the needed thermal cycles (soft bake). Appropriate 


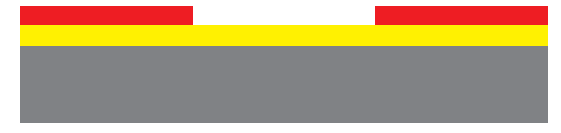

(a)

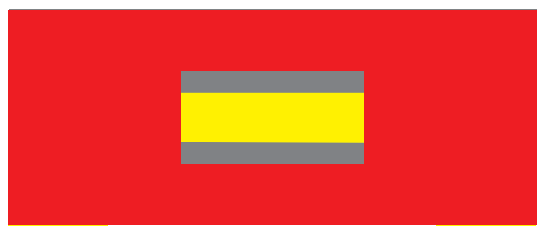

(b)

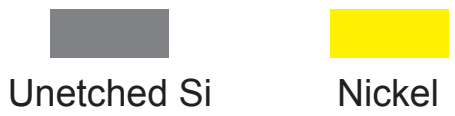

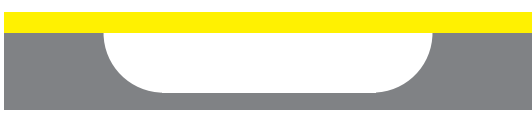

(c)

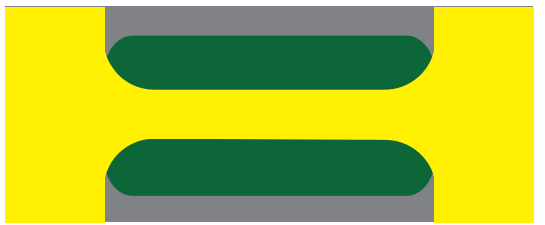

(d)

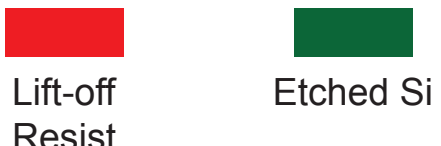

Figure 5.5: Illustration of fabrication method for freestanding Nickel beams. (a) Side and (b) top view of beam and photoresist mask prior to Silicon etch. (c) Side and (d) top view post-etch and lift-off resist removal.

etching procedures, either wet or dry, may be used to define beam geometries. Issues removing the AZ4210 resist are likely to occur, especially with RIE, so it may be advisable to follow the removal procedure of AZ4210 found below, prior to etching.

Electroplating requires a slightly different procedure and will be addressed briefly. This procedure is commonly used to create beams and films of thickness greater than one micron. The first step is deposition of a seed layer on top of the substrate. The seed layer is usually on the order of $\sim 100 \mathrm{~nm}$ and ideally is the same material that is being plated to assist in ease of removal. A positive PR stack is then patterned with mask 3 as seen in Figure 5.6. The PR should be at least as tall as the desired beam height as it defines the beam geometry. After the beams are plated to the desired thickness, the PR is removed with acetone and the seed layer is removed with an appropriate etchant. The resultant beam is a bilayer made of the seed layer and the electrodeposited layer. Process sheets for the electroplating process can be found in Appendix 8.2. 


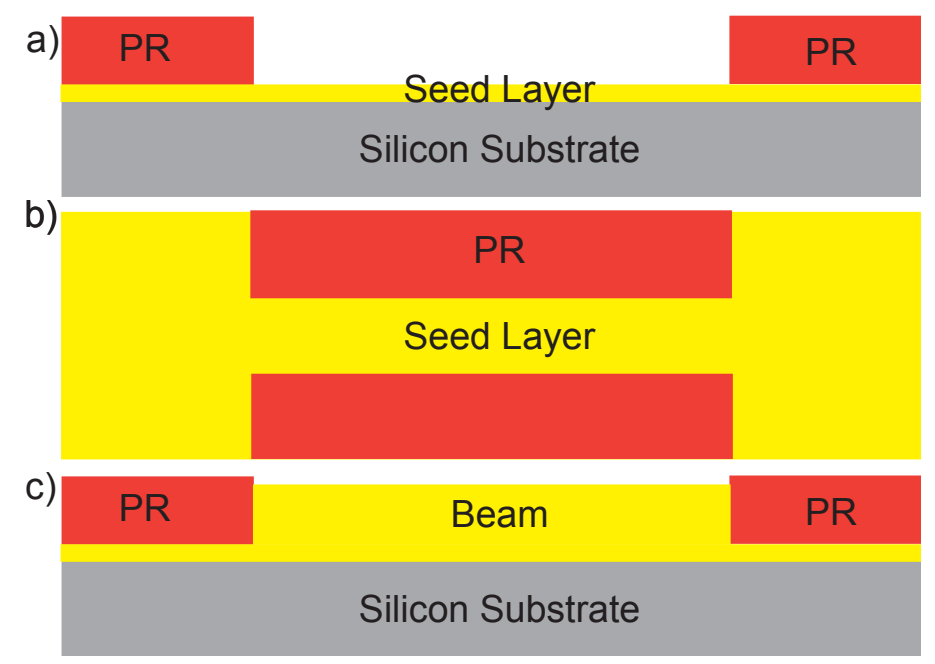

Figure 5.6: Side (a) and top (b) views of the lithography and seed layer prior to electroplating. (c) Plated structure prior to PR and seed layer removal.

\subsubsection{Annealing protocols}

Prior to beam release, annealing experiments were performed to determine the effects of processing temperature on the mechanical and microstructural properties of the film. Chips were cleaved from the 2-inch wafer into $\sim 1 \mathrm{~cm}$ x $1 \mathrm{~cm}$ squares. A total of nine chips were on every wafer. The annealing was performed on individual chips using an AXIC Inc. As-One rapid thermal processor (RTP) at 200 and $300 \mathrm{C}^{\circ}$ for one hour. The temperature ramp rate was $10 \mathrm{C}^{\circ}$ per second. Once the chamber temperature dropped below $40 \mathrm{C}^{\circ}$ the chips were removed. Samples were then tested to determine changes in geometrical, microstructural and mechanical properties due to this annealing treatment and are described in more detail in the sections and chapters to follow.

\subsubsection{Etching}

The result of the above fabrication steps are patterned beams on silicon substrates similar to figure 5.9. A novel mask design is used in conjunction with xenon $\operatorname{diflouride}\left(\mathrm{Xe} F_{2}\right)$ to release the beams from the silicon substrate. All etching was performed with a Xactix $\left(\mathrm{e}^{T M}\right.$ etcher. $\mathrm{Xe} F_{2}$ is a gas phase etch which isotropically etches silicon. The etch rate of $\mathrm{XeF}_{2}$ is dependent on the amount of exposed silicon and is governed by the reaction 


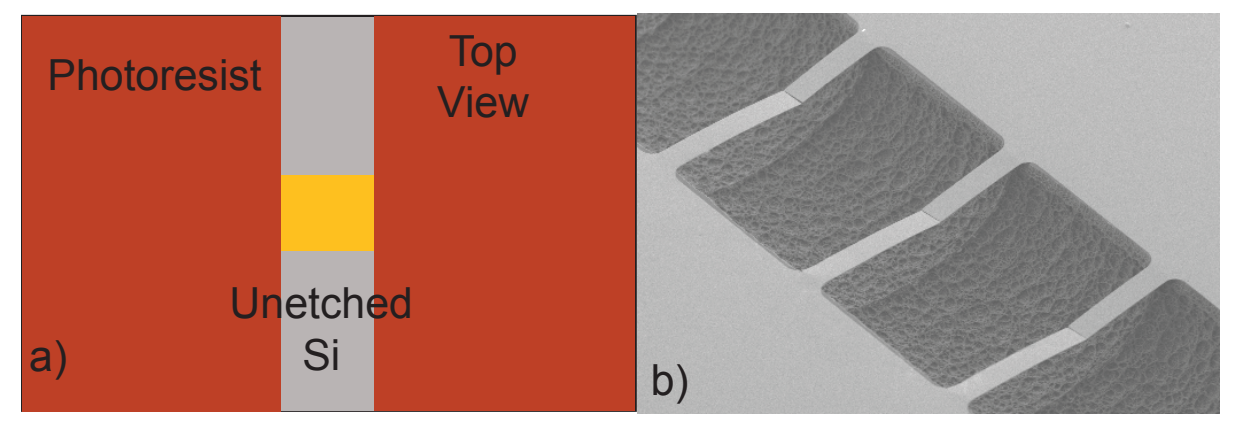

Figure 5.7: Original mask design (a) resulting in beams across an etched trench (b).

equation[44-46]:

$$
2 \mathrm{XeF}_{2}(\text { gas })+\mathrm{Si} \rightarrow 2 \mathrm{Xe}(\text { gas })+\mathrm{SiF}_{4}(\text { gas }) .
$$

In addition to selectively etching silicon it also attacks a variety of materials, including titanium. A large list of materials and their selectivity to etching by $\mathrm{Xe}_{2}$ can be found here[46].

Initial mask designs were a trench that ran along the length of the wafer similar to figure $5.7 \mathrm{a}$ which resulted in etched beams as shown in figure 5.7p. In order to isolate individual beams, the etch mask was changed to the design in figure $5.9 \mathrm{a}$, which results in the final etch configuration in figure 5.9p and c. The edge of the etch mask was designed to be 60 $\mu \mathrm{m}$ from the clamped edge of the beam so the etch would sufficiently etch out the trench under the beam while etching toward the edge. There were a number of samples that failed in the early stages of this project which led us to believe the global bending stiffness of the entire chip was being reduced by the etch trench. This stiffness reduction would allow the chip to bow, imparting undesired stress on the released beams. These beam failures ended up being caused by low quality sputtered films near the end of the target's life which cracked upon deposition as seen in Fig 5.8 .

To define the etch area a second bilayer photoresist film, identical to the first described 


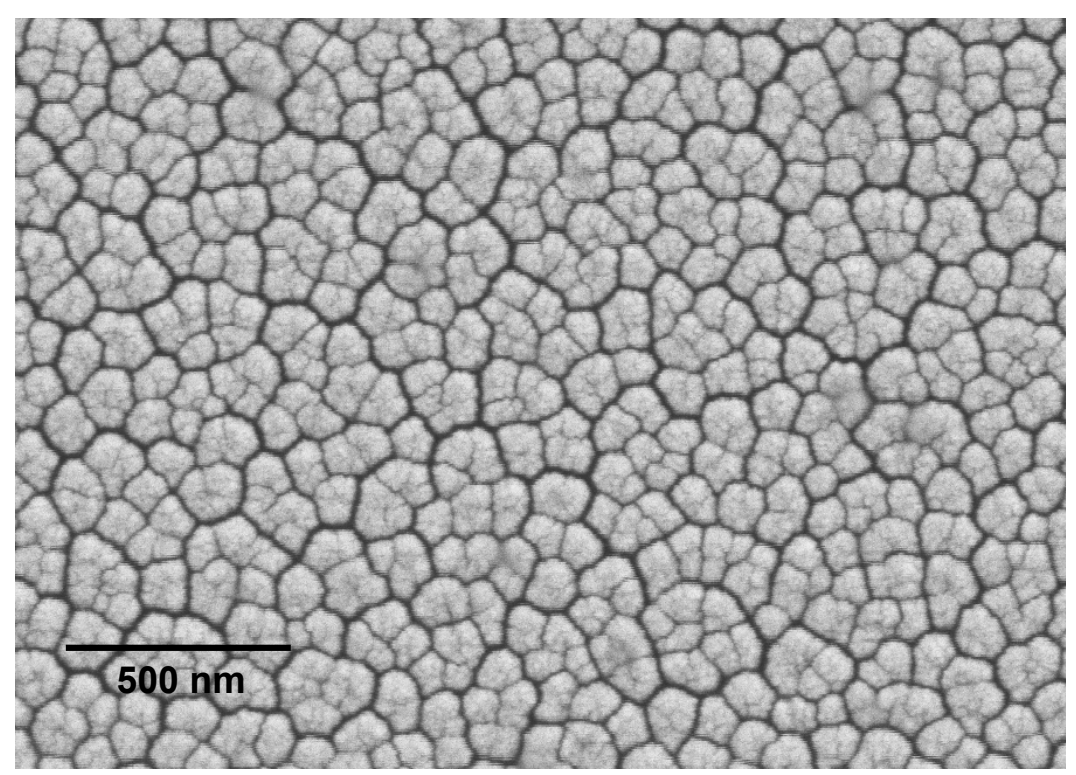

Figure 5.8: Films with microcracks from early beams where visible microcracks caused low beam yield from fabrication, low strength and reduced effective modulus when tested.
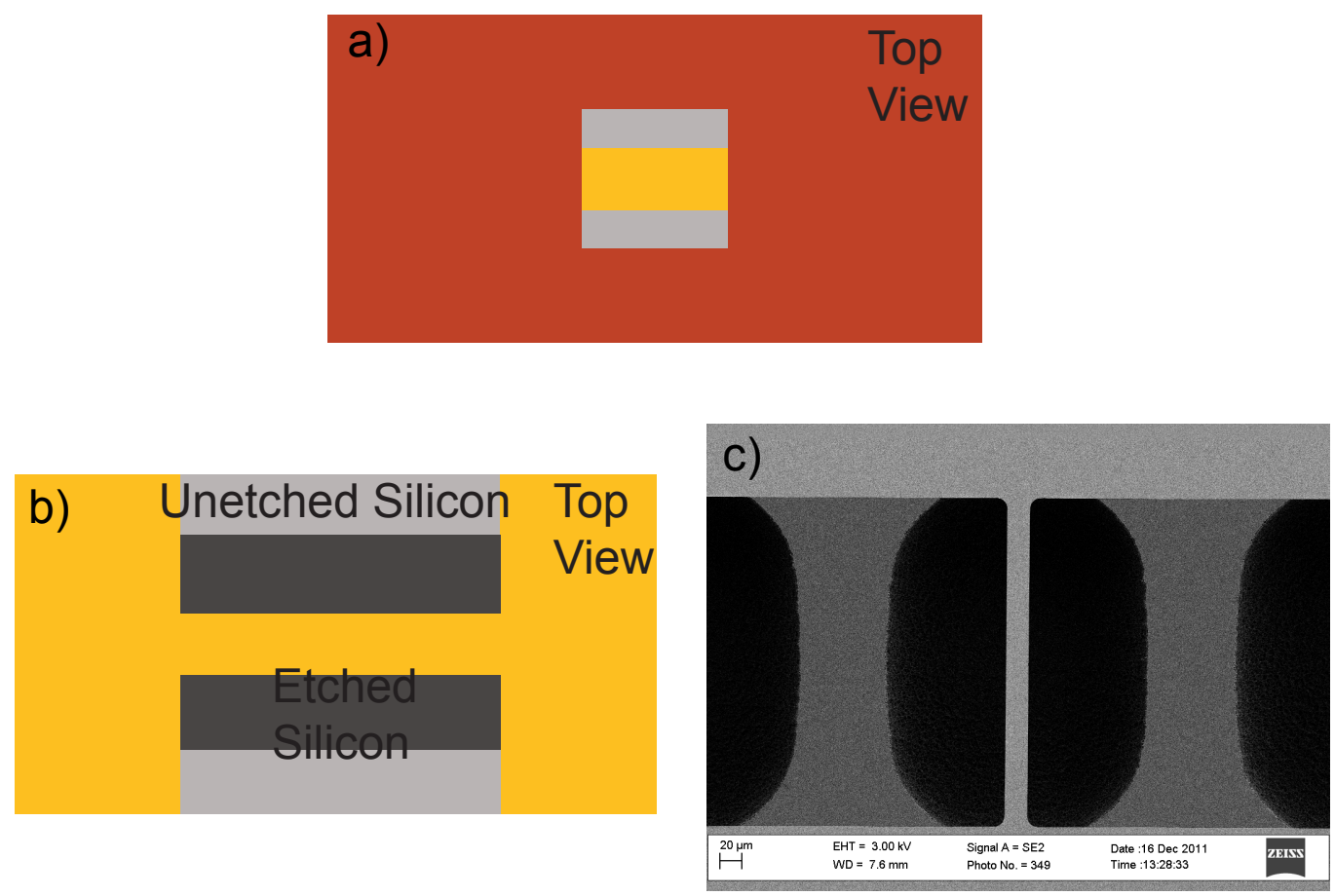

Figure 5.9: Photoresist mask prior to etching (a), representation of etched beam (b) and SEM of $300 \mu \mathrm{m}$ long by $20 \mu \mathrm{m}$ wide beam showing unetched silicon supports (c). 


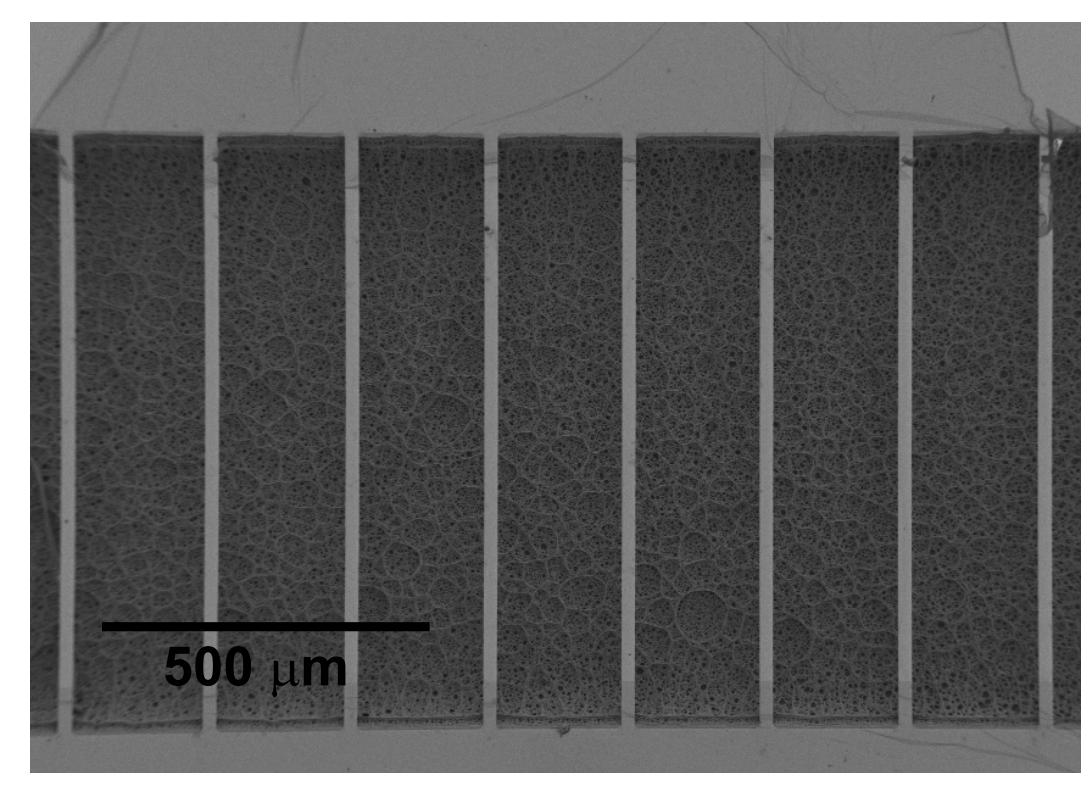

Figure 5.10: Residual PR remaining on released beams.

above, was patterned as shown in Figs. 5.5 a and 5.5p. After development the top layer, AZ4210, was removed with acetone leaving the LOR10B. This relatively minor step turned out to be a pivotal advancement in the fabrication procedure. During the evolution of the fabrication process it was determined that AZ4210 hardens during the etch phase and became too difficult to remove without a high temperature NMP bath, which destroyed the beams. An example of the residual AZ4210 can be seen in Figure 5.10 on 500 $\mu \mathrm{m}$ long beams. The silicon and titanium layer beneath the beam were removed using an $X$ etch ${ }^{\circledR}$ e1 etcher by cycling 2 mTorr of $\mathrm{XeF}_{2}$ gas into the etching chamber for two minutes per cycle. The beams were inspected via optical microscopy after three etch phases and every phase thereafter to determine when the etch was complete. Beams were stopped before overetching occurred so the length of the beam could be determined. The LOR10B layer was removed after etching by soaking in a $\mathrm{KOH}$ based developer, AZ400K, for an hour or until the LOR10B is fully removed. Figure 5.9a and Figs. 5.5 $\mathrm{c}$ and 5.5 d show a beam post-release. The trench under the beams is on the order of $\sim 60 \mu \mathrm{m}$ which allows for large deflections without etching from the backside of the wafer. 


\subsection{Length-scale characterization}

The discussion of beam results in the following chapter requires beam dimensions to analyze the data. This section presents an overview of the methods, measurement techniques and resulting experimental errors, both geometric and microstructural, used in chapters to follow.

\subsubsection{Geometric characterization}

Thickness measurements were performed with a Veeco Dektak 8 mechanical profilometer that was calibrated on a $48 \mathrm{~nm}$ standard to within $\pm 0.5 \mathrm{~nm}$. Standard deviation in average beam thickness for a set of five beams is the average of one measurement on each beam in a set. The thickness measured is the combined thickness of the nickel beam film and the titanium adhesion layer. Because the titanium layer is removed during the etch phase it is taken as $9 \mathrm{~nm}$ and subtracted from the thickness measurement to obtain the thickness of the nickel film. The titanium film thickness was taken as $9 \mathrm{~nm}$ even thought the optimal thickness of the titanium film was 10nm. During the evaporation process, the thickness of the deposition was monitored by a quartz crystal microbalance and the final total film thickness, measured via mechanical profilometry, was consistently lower than the desired thickness. The standard deviation for all beam sets is taken as $\pm 1 \mathrm{~nm}$ which takes into account the uncertainty from the thickness of the titanium film as well as deviations due to surface roughness. In order to ensure that the profilometry measurement did not damage the beams, measurements were taken on the set of beams with dimensions $20 \mu \mathrm{m}$ in width by $300 \mu \mathrm{m}$ in length with a stylus force of $1 \mathrm{mg}$, the lowest setting. These beams are in close proximity to the beams tested $(20 \times 200 \mu \mathrm{m})$ and as such were considered a representative sample.

Width and length measurements were performed via SEM using a Zeiss FESEM SUPRA 40 instrument. A working distance of $\sim 8 \mathrm{~mm}$ was used and then the beams were zoomed 

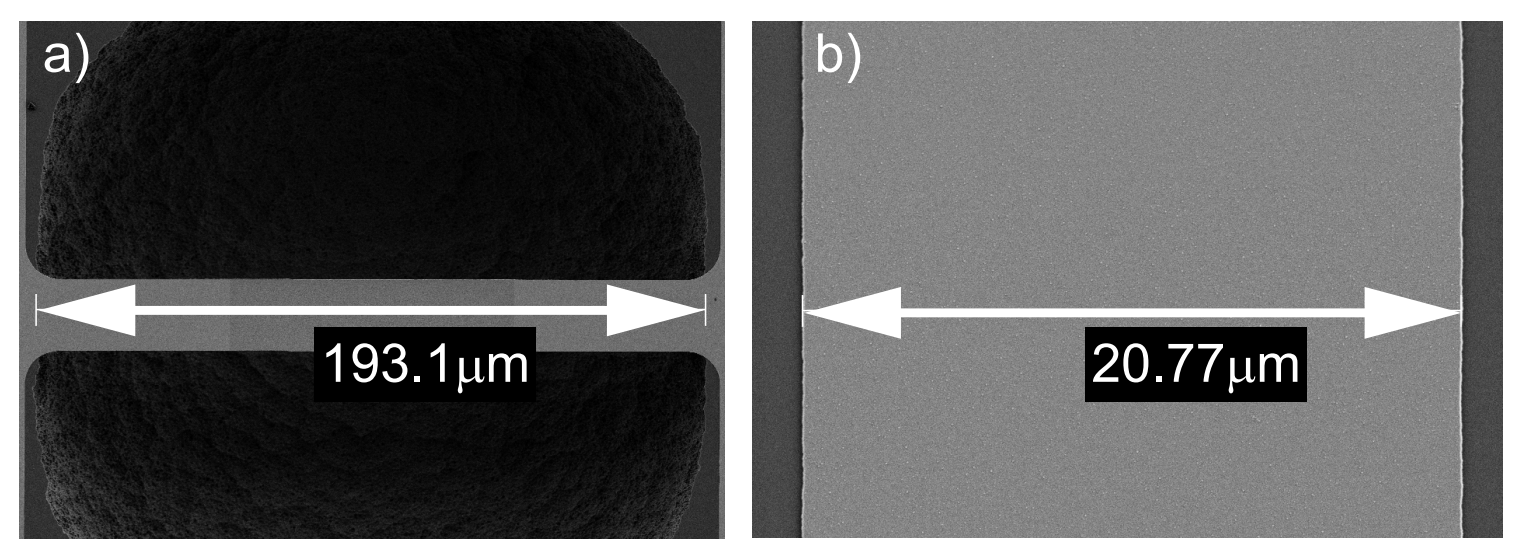

Figure 5.11: a) Length measurement of $81 \mathrm{~nm}$ thick released beam. b) Width measurement of released beam post thermal treatment, before etching.

in on as much as possible while keeping the relevant dimension in view. The instruments measurement tool was used to measure dimensions. Figure 5.11 shows a representative measurement on an $81 \mathrm{~nm}$ thick $200 \mu \mathrm{m}$ by $20 \mu \mathrm{m}$ beam. Again the standard deviations in average width and length are from one measurement on each beam in a set. In general most beams etched to the same approximate length with a few anomalies most likely due to residual dirt or PR remaining on the surface of the silicon. For the sake of accuracy, all results in the following chapter use the actual beam length instead of the average value for the set. For all results in the following chapter where width and thickness are a variable in single beam calculations, the average value of both are used as the width and thickness only vary slightly across the length of the beam (and wafer).

The length of the beam was confirmed via optical profilometry using a Wyko NT1100 optical profiler. Figure 5.12 a shows a top view contour of the beam. Because the etch starts from the sides of the beams and works in, there is a slight curl across the width of the beam in the center of the beam. For all beams this curl is less than one micron and does not affect indentation testing. Figure $5.12 \mathrm{p}$ shows the raw data along the length of the beam that is used to calculate the length for the same beam measured in Fig. 5.11a. The data shown is the average of a ten micron wide section along the length of the beam. The sharp 


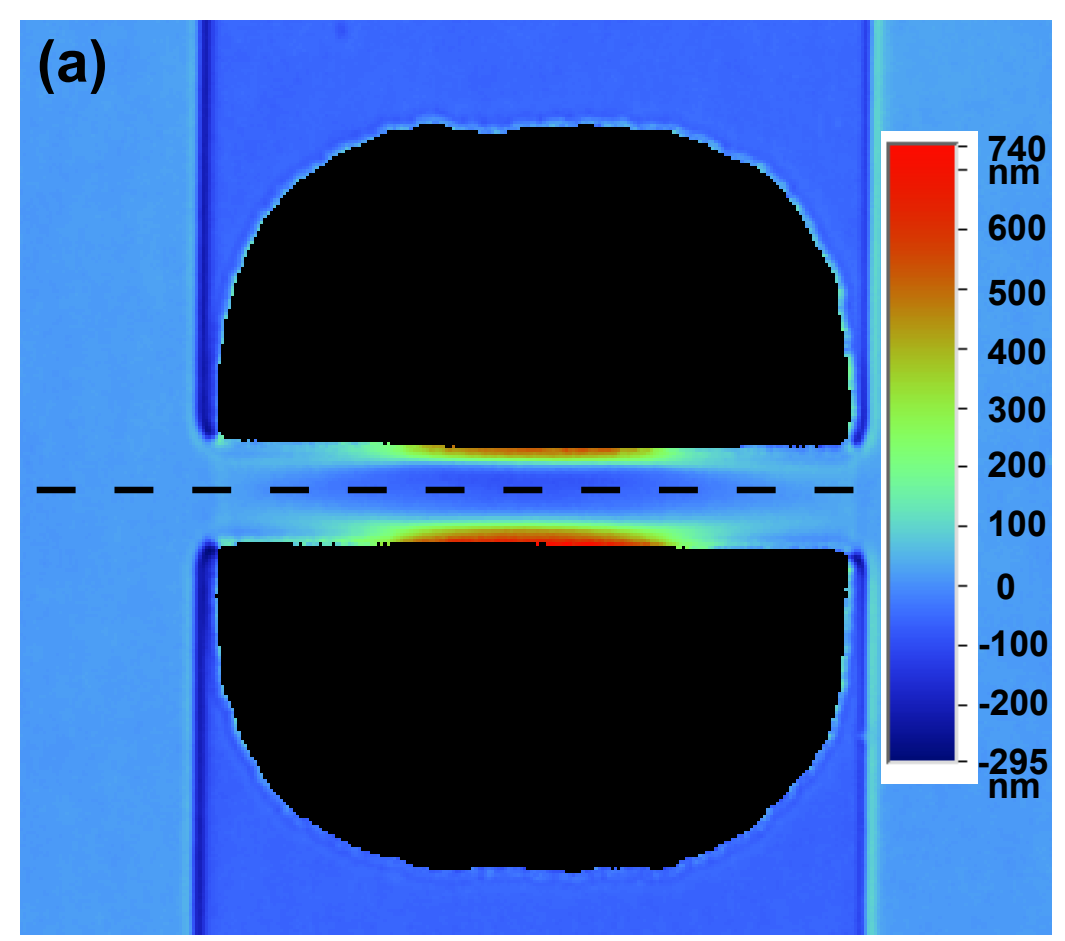

(b)

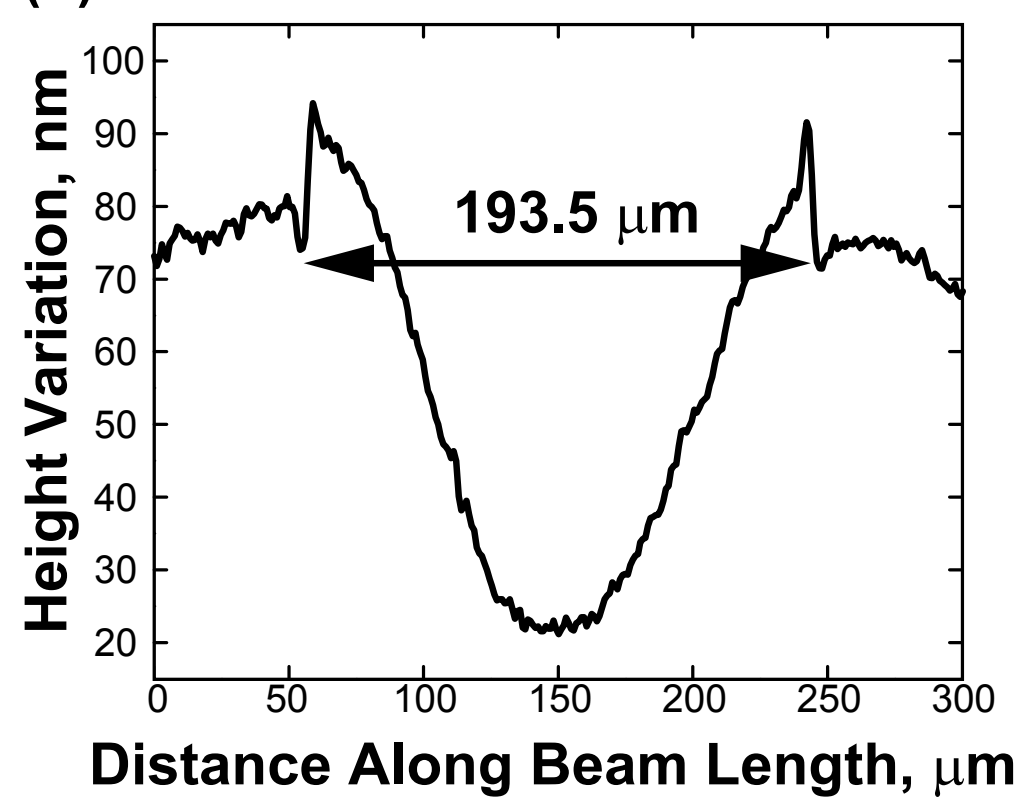

Figure 5.12: a) 2D contour map of beam where the difference between the darkest blue and red contours on the beam is less than one micron. Dashed line across beam center shows 2D cross-section seen below. b) 2D raw data along length of beam using the abrupt change in height due to the interface as the definition for the length of the beam. 
transition on either side of the beam designates the silicon-nickel interface (i.e. a change in the interference patern) and agrees well with the SEM data. The same beam in Fig. 5.11 shows a length of $193.1 \mu \mathrm{m}$. A standard deviation of $\pm 0.5 \mu \mathrm{m}$ was chosen which consistently captured the differences between the WLI and SEM measurements. Beams lengths were triple checked via the optical microscopy stage on the nanoindentation system prior to testing.

\subsubsection{Microstructural characterization}

Average grain size, crystallite size and crystal orientation data were collected via X-ray diffraction and SEM. High magnification SEMs of the as-deposited and 200C annealing condition were used to determine approximate grain size using the image processing software ImageJ[47]. It was very difficult to get clear SEMs at the scale needed to automate the entire process (i.e. crisp definition of grain boundaries) so each grain was hand drawn to clearly define the boundary between grains as seen in Fig. 5.13a. After assigning a pixel value to the length of the scale bar and converting the image to a binary image, as in Fig. $5.13 \mathrm{p}$, Image $\mathrm{J}$ is then able to process the area of the outlined grains. Because we assume the grains to be approximately spherical, the diameter of each grain is then converted using the equation for the area of a circle. For each of the as-deposited and 200C anneal conditions for each film thickness at least 100 grains were analyzed.

It was not possible to analyze the 300C annealed condition via SEM because the grains appeared to coarsen and it was impossible to pick individual grains apart. An example SEM of a 300C annealed condition is seen in Fig. 5.14 where various portions of the film appear to be starting to coalesce into larger grains. The smaller crystallites are still evident on the surface even with the appearance of the larger grains forming. In order to have a consistent picture of the grain size and crystallite distribution of all the films, the average crystallite

sizes were analyzed via X-ray diffraction (XRD) as a comparison to the SEM analysis. The 
(a)

(b)

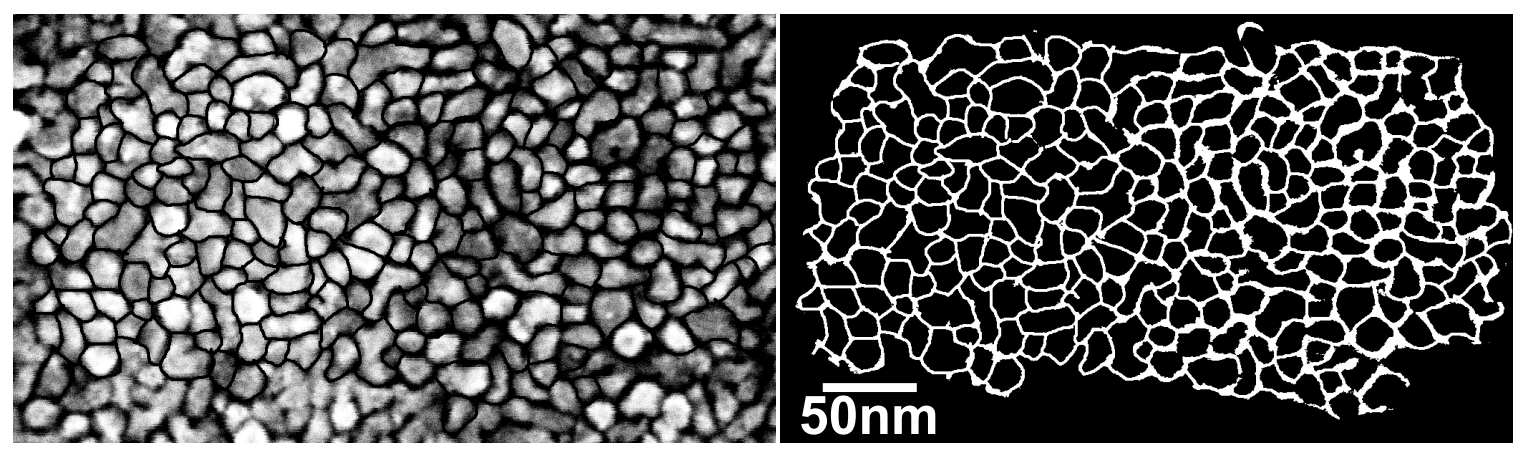

Figure 5.13: a) Outlined grains allowing image to be converted to a binary image. b) Binary image which allows for automated grain size analysis.

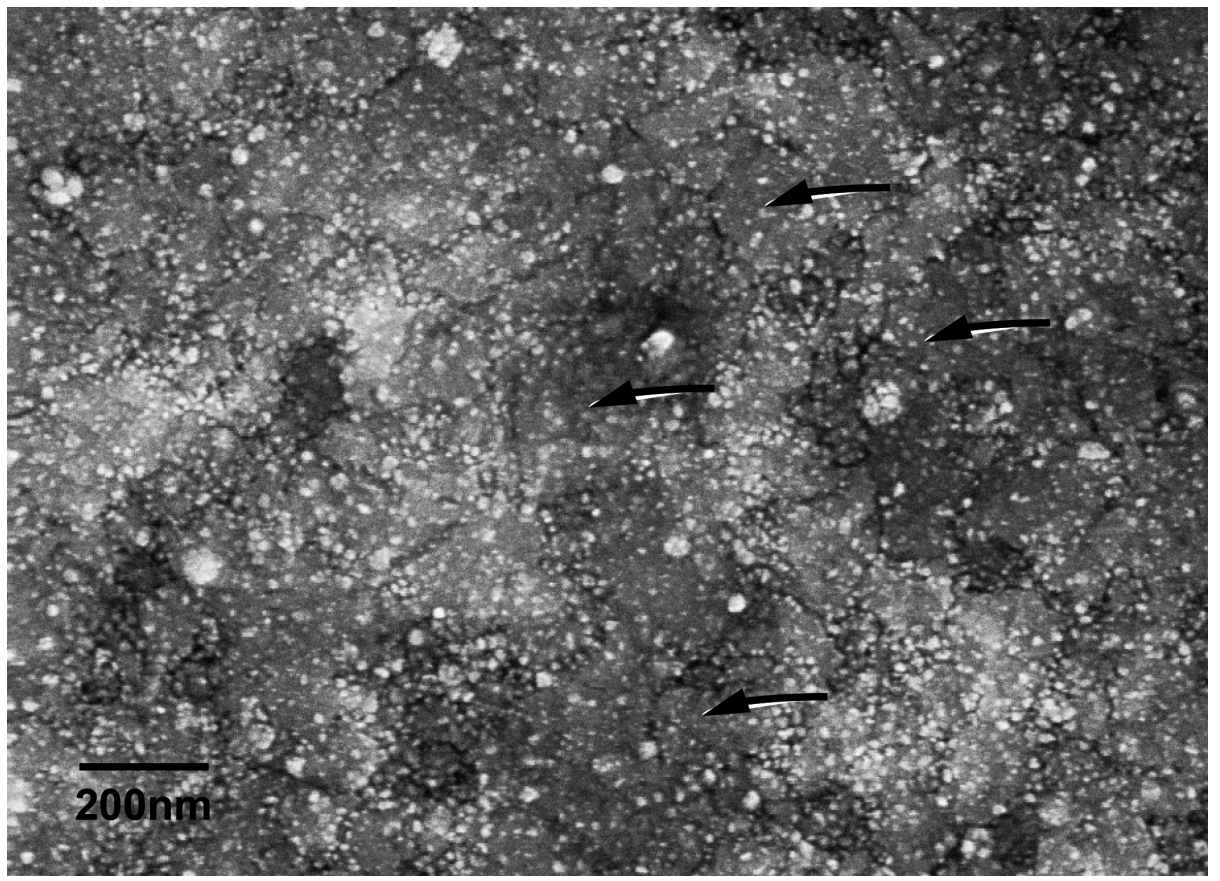

Figure 5.14: $232 \mathrm{~nm}$ thick film annealed at 300C for one hour. Larger grains appear to be forming, indicated by black arrows, while evidence of the smaller grains seen in the previous annealing conditions still exist. 
grain size from SEMs and the crystallite size are of the same general magnitude for the asfabricated and $200 \mathrm{C}$ annealed cases. Although it was not possible to get a quantitative grain size measurement from the $300 \mathrm{C}$ case from SEM, it is clear that there is a wide variety of grain sizes from tens of nanometers to hundreds of nanometers. The assumption is taken that XRD crystallite size deviates from the actual grain size at sizes larger than about a hundred nanometers. Previous work has shown good agreement between XRD results and TEM grain counting studies so we will assume the XRD results for the as-fabricated and $200^{\circ} \mathrm{C}$ are the actual grain size which is reinforced by the SEM results[48 -51$]$.

The X-ray diffraction measurements were taken with a Rigaku SmartLab ${ }^{\circledR}$ using $\mathrm{Cu}$ X-rays. A Germanium double bounce $\left(\begin{array}{lll}2 & 0 & 0\end{array}\right)$ monochromator was used to strip the $\mathrm{Cu}$ $K \alpha 2$ x-rays. Although the monochromator does not completely remove the $K \alpha 2$ radiation, it is $99 \%$ effective and thus the effects of $K \alpha 2$ radiation in the analysis is ignored. The diffractometer was operated at $40 \mathrm{kV}$ and $44 \mathrm{~mA}$ in continuous mode with a scan speed of 1 degree/minute. In order to extract the necessary data to determine the crystallite size the (1 11 ) diffraction peak, such as those seen in figure 5.15a, is fit to a Gaussian distribution. The Gaussian distribution is represented by

$$
y=y_{o}+\frac{A}{\beta \sqrt{\frac{\pi}{2}}} e^{-2 \frac{\left(x-\theta_{0}\right)^{2}}{\beta^{2}}}
$$

where $y_{o}$ represents the background noise in the data, $\beta$ is the full width of the Gaussian fit at half the maximum intensity, $\mathrm{A}$ is the area under the curve and $\theta_{o}$ is the Bragg angle where the incident $\mathrm{x}$-ray radiation interferes constructively with a given crystal plane creating a maximum signal. All film thickness/annealing combinations were highly textured in the (1 11 ) direction. This can be seen in the representative XRD measurements in figure $5.15 \mathrm{~b}$. Using the Scherrer formula[52, 53]

$$
D_{\text {grain }}=\frac{K \lambda_{K \alpha 1}}{\beta \cos \left(\theta_{o}\right)}
$$




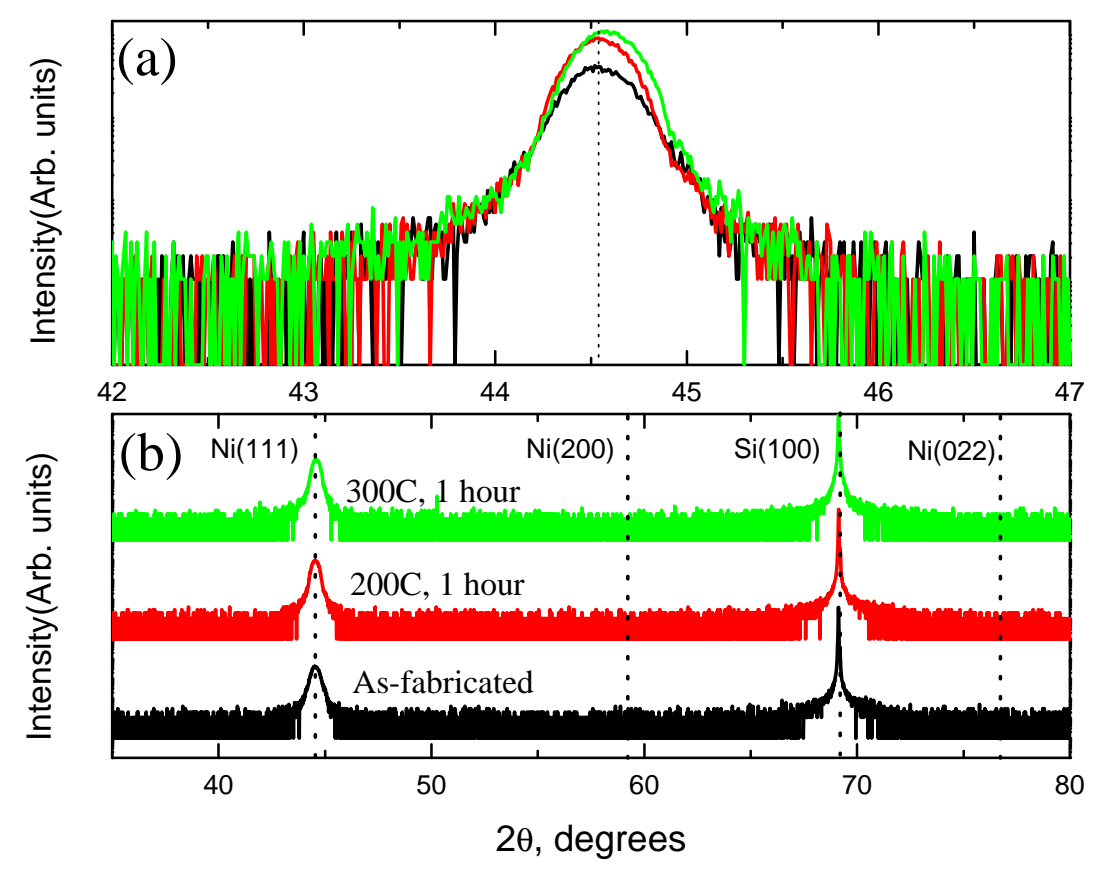

Figure 5.15: Nickel (111) peak for all thermal treatments (a) the full x-ray diffraction spectrum showing a highly textured film in the $\left(\begin{array}{lll}1 & 1 & 1\end{array}\right)$ direction as well as the $\left(\begin{array}{lll}1 & 0 & 0\end{array}\right)$ silicon peak.

where $D_{\text {grain }}$ is the diameter of the crystallite size with an assumed spherical shape, $\mathrm{K}$ is a dimensionless shape factor taken as 0.9 for spherical crystals and $\lambda_{K \alpha 1}$ is the wave length of the $\mathrm{Cu} X$-ray with the $K \alpha 2$ radiation stripped off. The results, presented in Table 5.1, will be discussed in the following chapter with respect to mechanical properties of these nickel films. The XRD results are used for quantitative comparisons in the for the as-fabricated and $200^{\circ} \mathrm{C}$ films. 
Table 5.1: Nickel Grain and Crystallite Size

\begin{tabular}{ccc}
\hline \hline Beam Set & SEM Grain Size $(\mathrm{nm})$ & XRD Crystallite Size $(\mathrm{nm})$ \\
\hline 42nm As-Deposited & $12.4 \pm 3.9$ & 16.7 \\
42nm 200C & $15.5 \pm 3.8$ & 17.1 \\
42nm 300C & - & 17.5 \\
81nm As-Deposited & $14.9 \pm 3.9$ & 17.9 \\
81nm 200C & $15.4 \pm 3.6$ & 20.4 \\
81nm 300C & - & 21 \\
232nm As-Deposited & $22.1 \pm 5.3$ & 20.7 \\
232nm 200C & $22.9 \pm 5.0$ & 23.5 \\
232nm 300C & - & 23.1 \\
377nm As-Deposited & $25.4 \pm 6.6$ & 19.1 \\
377nm 200C & $25.2 \pm 5.7$ & 24.9 \\
377nm 300C & - & 23.5 \\
\hline \hline
\end{tabular}




\section{Chapter 6}

\section{Elastic-plastic Properties of Nickel}

\section{Beams}

The objective of this chapter is to provide a cohesive example of test theory, interpretation and fabrication laid out in the preceding chapters. Nickel beams of thickness 42 , 81, 232 and 377nm were fabricated using the procedure outlined in Chapter 5. The beams were in either their fabricated state or were annealed for one hour at 200 or $300{ }^{\circ} \mathrm{C}$ prior to release. This annealing, as well as heat seen during the fabrication process, resulted in variations in the initial stress state of the beam as well as alterations of the microstructure of the films via grain growth. The effects of both annealing temperature and film thickness are presented below using the interpretations laid out in Chapter 3. Beams were tested via center span point-loaded indentation using the methods from Chapter 2. The results show the fabrication procedure, testing method and data analyses presented may be used to investigate a host of different mechanical behaviors including modulus, residual strain, yield stress and fracture properties. 


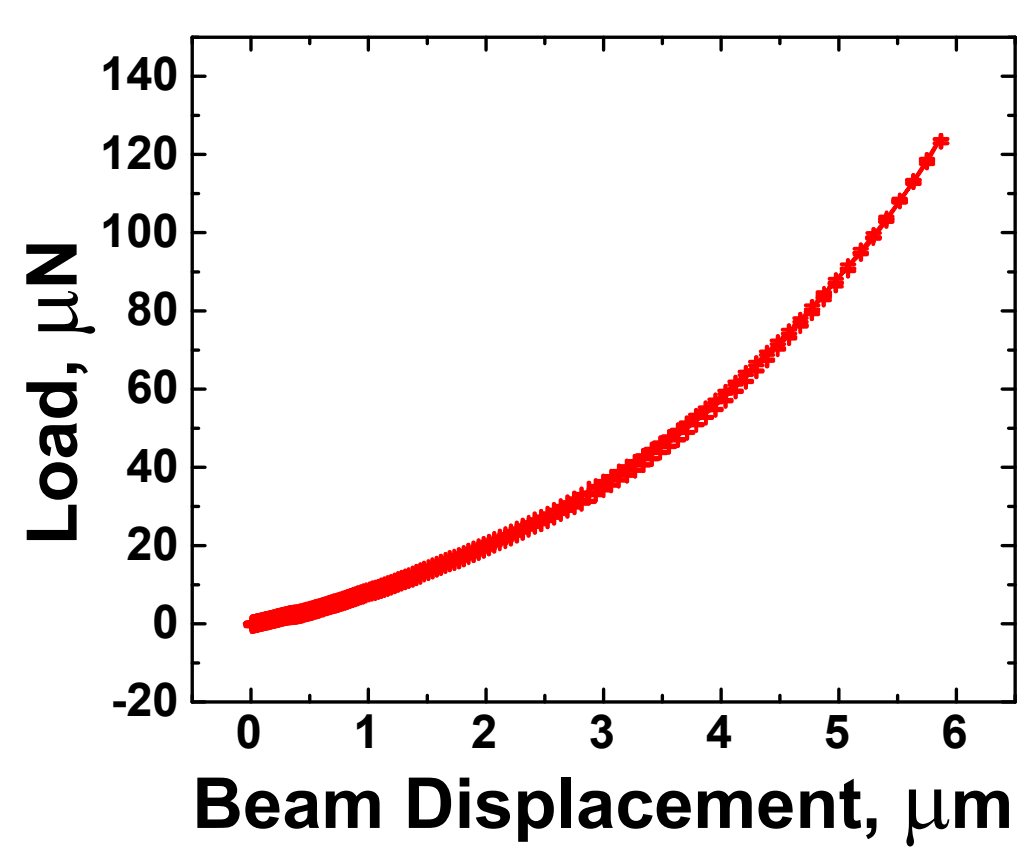

Figure 6.1: Load and unload curves on $81 \mathrm{~nm}$ thick unannealed beam where the maximum displacement corresponds to a strain of 0.002. Standard deviations are the average of 6 tests on the same beam showing identical behavior in the load and unload portions.

\subsection{Test parameters and protocols}

Beams with thickness 42, 81, 232 and 377nm (full dimensions can be found in tables 8.2, 8.3, 8.4 and 8.5 were probed in the center of their spans using the indenter equipped with a $50 \mu \mathrm{m}$ radius Sapphire sphere. Three different beams from each thickness and annealing combination were tested. The transition point from the ramp loading in the elastic regime to constant strain rate prior to the plastic regime was chosen as $\varepsilon=0.001$, which is equivalent to $4.5 \mu \mathrm{m}$ for a beam of length $200 \mu \mathrm{m}$. Using either figure 2.17 or eqn. 2.23 , the error between the prescribed strain rate, $\dot{\varepsilon}=0.0001 s^{-1}$ for all tests, and the actual strain rate is shown to be approximately $2 \%$ at the transition point. It is important to note this error continues to decrease as the beam displacement increases and quickly becomes less than one percent.

In order to examine the effects of thermal drift in the nanoindenter column on load- 
displacement behavior, a series of six tests on a single $81 \mathrm{~nm}$ as-fabricated beam were performed over 120s as shown in figure 6.1. All tests were performed after the system settled to a drift of $<0.05 \frac{\mathrm{nm}}{\mathrm{s}}$. The load and unload curves show nearly identical behavior suggesting entirely elastic behavior below $\varepsilon=0.002$. There is a small load offset of 490 $\mathrm{nN}$ at the completion of the tests in figure 6.1 which is attributed to drift in the system. Taking the drift rate as $0.05 \frac{\mathrm{nm}}{\mathrm{s}}$ gives a total displacement drift of $6 \mathrm{~nm}$ over the span of the test(120s), which is clearly negligible compared to the maximum beam displacement of $\sim$ $6 \mu \mathrm{m}$. Multiplying by the column stiffness of $\approx 82 \frac{\mathrm{N}}{\mathrm{m}}$ gives a theoretical load drift of 492 $\mathrm{nN}$ which corresponds well to the total drift seen experimentally. It becomes evident that the load accrued from thermal drift is negligible for a test of this length as the contribution of the drift load to the total load at the maximum displacement is $<0.2 \%$.

An examination of the $42 \mathrm{~nm}$ thick unannealed condition, which is the film and thermal treatment state most susceptible to the effects of thermal drift as it is the most compliant beam, show total test times in the range of 360-390s and maximum loads from $420-500 \mu \mathrm{N}$. At these times and loads, the contribution of drift load to the total load is on the order of $<0.3 \%$ and thus is considered negligible. Taking into account thicker films and annealed films will produce stiffer responses for the same general testing time scales (i.e. the same approximate total strains), it is safe to assume thermal drift can be ignored for all presented test results.

The load-deflection response of the $81 \mathrm{~nm}$ beam in figure 6.1 sheds light on a few other important details of these tests. The repeatability of the tests and similarity between the loading and unloading curves suggests that anelastic (time dependent) deformations are not present over this time scale. Additionally, the repeatability of the data over six tests points to the absence of inelastic deformations, meaning under a strain of $0.2 \%$ the entire test remains in the elastic regime. It should be noted this series of tests was only performed on the $81 \mathrm{~nm}$ unannealed sample and this observation in particular may not (and appears 


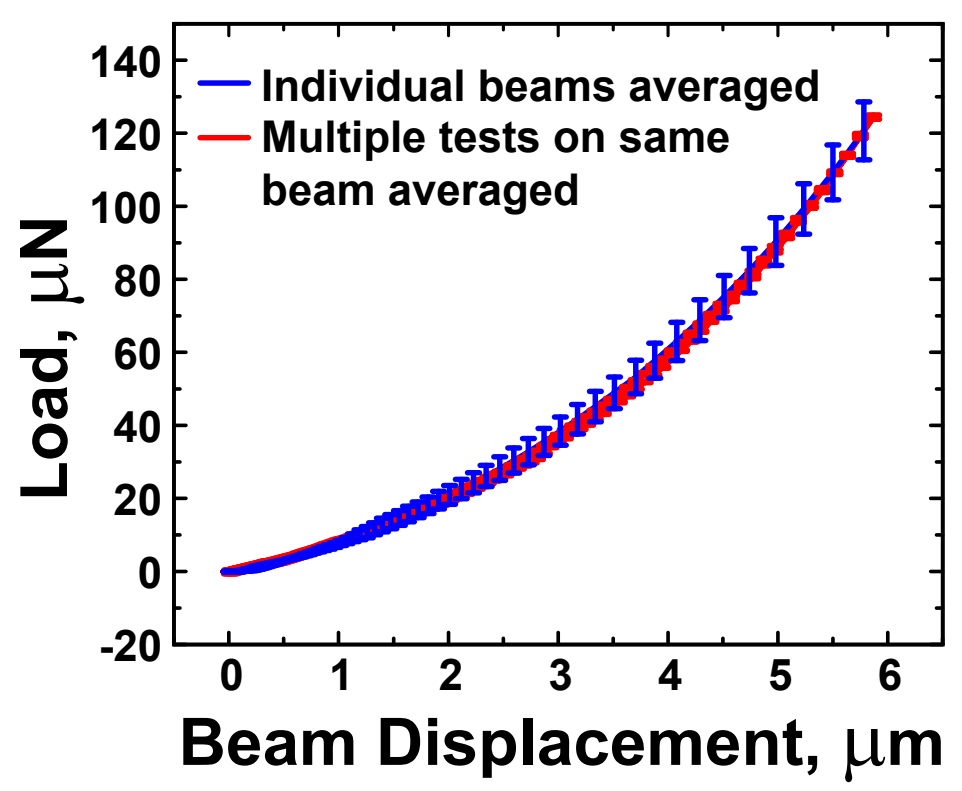

Figure 6.2: Loading curves for (red) multiple test on the same beam averaged and (blue) three different beams averaged together. The similarity between curves suggests excellent repeatability from beam to beam and suggests the first loading to be indicative of the true response of the beam.

not to) hold true for all other cases. In hindsight it would have been advisable to examine repeated loading on a beam in each set to determine elasticity at small deflections.

Repeatability of results from beam to beam is demonstrated by examining the average of individual tests on multiple beams to the average of multiple tests on the same beam as seen in figure 6.2. The average of multiple tests on the same beam appears to have little to no variation from the average of tests on individual beams. It should be noted the loaddisplacement curves seen in figure 6.2 do not take into account the difference in length from beam to beam, in this case all beams are within a micron in length of each other, which likely accounts for the error in the measurement (i.e. a longer beam will exhibit a more compliant load deflection curve). 


\subsection{Data post-processing}

All figures in this section used to show examples of post-processing are from the same $81 \mathrm{~nm}$ as-fabricated beam unless explicitly stated. In order to extract the elastic modulus, unloading segments were inserted at specified strain intervals. For the as-fabricated and $200^{\circ} \mathrm{C}$ anneal cases these intervals were at $\varepsilon=0.005,0.0075$ and 0.01 , unloading by a strain of 0.002 (i.e. the unload segment from $\varepsilon=0.005$ unloads down to $\varepsilon=0.003$ then begins to reload) which is clearly demonstrated in figure $6.3 \mathrm{a}$. For the $300^{\circ} \mathrm{C}$ anneals the unloads were performed at $\varepsilon=0.003$ and unloaded to $\varepsilon=0.002$, as the fracture stress/strain levels were much lower for these cases and this strain level assured an unload segment prior to fracture. While the analysis provided in Chapter 3 allows for the determination of modulus and residual strain through fitting of the normalized load-deflection curve, here the elastic modulus is determined from the normalized unloading curve in the membrane regime (or very close to the membrane regime) where the approximation variable $\mathrm{c}=2$. This approach is taken primarily because: (i) the unloading curve will be absent of any inelastic deformations and (ii) the solution becomes exact in the membrane regime. A representative stress strain curve can is seen in figure 6.3 a showing the full range of a test.

Using the elastic modulus from the unloading curves the residual strain was computed from the loading curve using eqn. 3.36 with $\mathrm{c}=2.12$. The residual strain is computed between $\varepsilon=0.0001-0.001$. This range was chosen in an effort to ensure that the beam is uniformly loaded on the lower end and stays away from a strain regime where permanent deformation may be in play on the upper end. Figure 6.3 b shows a representative normalized load vs. displacement curve, noting that the area fitted for residual strain in this particular case is from $\Delta \approx 15-55$.

At this point it is useful to recognize that the residual strain in the beam presents itself in the stress strain curve as the intercept of the stress axis (i.e. residual stress). The entire 

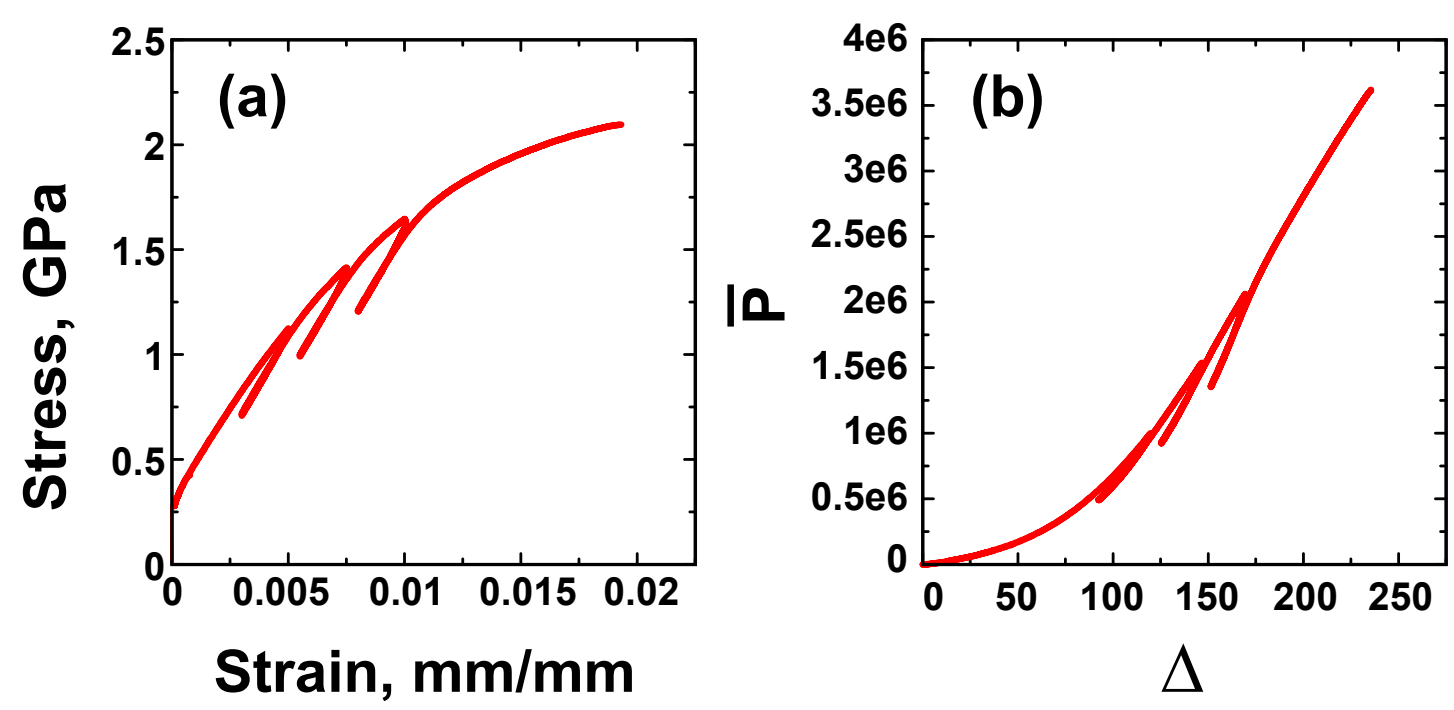

Figure 6.3: Stress strain behavior (a) and normalized load vs. displacement(b)

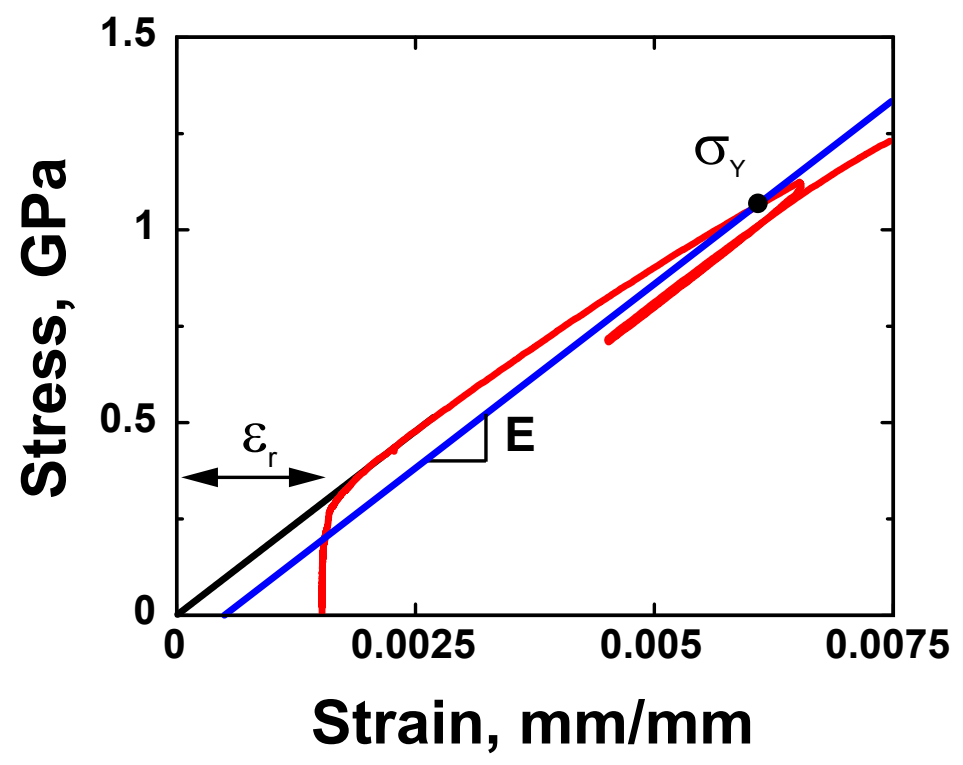

Figure 6.4: Example of the procedure used to create stress strain curves and determine the flow stress. The black line is the linear extrapolation of modulus, the red line is the original stress strain curve which has been shifted to the right by an amount equal to the residual strain and the blue line is the modulus offset by $0.05 \%$ strain which is used to determine the flow stress. 
stress strain curve starting at zero stress and strain was created by shifting the stress strain curve to the right by an amount equal to the residual strain and extrapolating a line from the origin to the shifted curve with a slope equal to the elastic modulus. This extrapolated line fits the calculated residual strain within $10 \%$ in all cases. An example of this procedure is seen in figure 6.4 and also shows the modulus offset by a strain of $0.05 \%$ which is used to calculate the flow stress. This offset strain was chosen because it allows for determination of a flow stress in all cases, even those where there appears to be very little ductility before fracture which can be seen in figure 6.13 in the 42 and $81 \mathrm{~nm}$ thick films for the $300^{\circ} \mathrm{C}$ annealed case. The fracture stress is simply the stress at fracture and the fracture strain is defined as the strain at fracture after the stress strain curve has been shifted. It is important to note this procedure is done on each individual beam's data set and the individual properties are extracted and then averaged together before averaging the data together to get average stress strain curves shown in figure 6.13. All of the raw properties from each thickness/annealing combination can be found in appendix 8.4.

\subsection{Results}

\subsubsection{Residual Strain}

Residual strain results as a function of film thickness for each annealing condition are shown in figure 6.5. Examining the as-fabricated and $200^{\circ} \mathrm{C}$ cases, there appears to be little difference between the two. If the assumption is made that the residual strain is purely from mismatch of thermal expansion coefficients, the three dashed lines in figure 6.5 represent the maximum temperature seen by each of the three thermal conditions (the asfabricated sample sees $160^{\circ} \mathrm{C}$ for five minutes after deposition). The deposition temperature is assumed to be at room temperature $\left(\sim 30^{\circ} \mathrm{C}\right)$ and the mismatch in coefficients of thermal expansion between nickel and silicon is $10 \times 10^{-6} /{ }^{\circ} \mathrm{C}$. This thermal mismatch strain is 


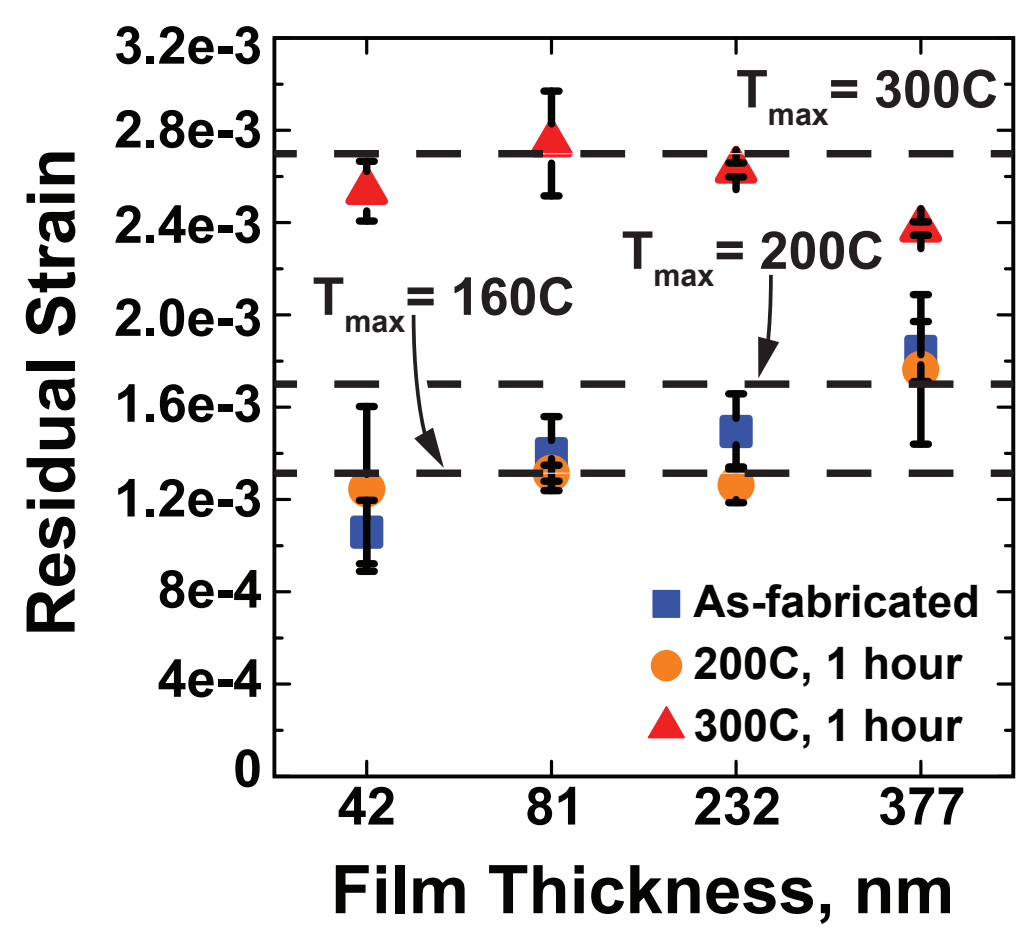

Figure 6.5: Residual strain shows little variation between the as-fabricated and $200^{\circ} \mathrm{C}$ anneal but sees a large jump at $300^{\circ} \mathrm{C}$. The dashed lines represent the expected residual strain due to thermal mismatch strains where the deposition temperature is assumed to be room temperature. 


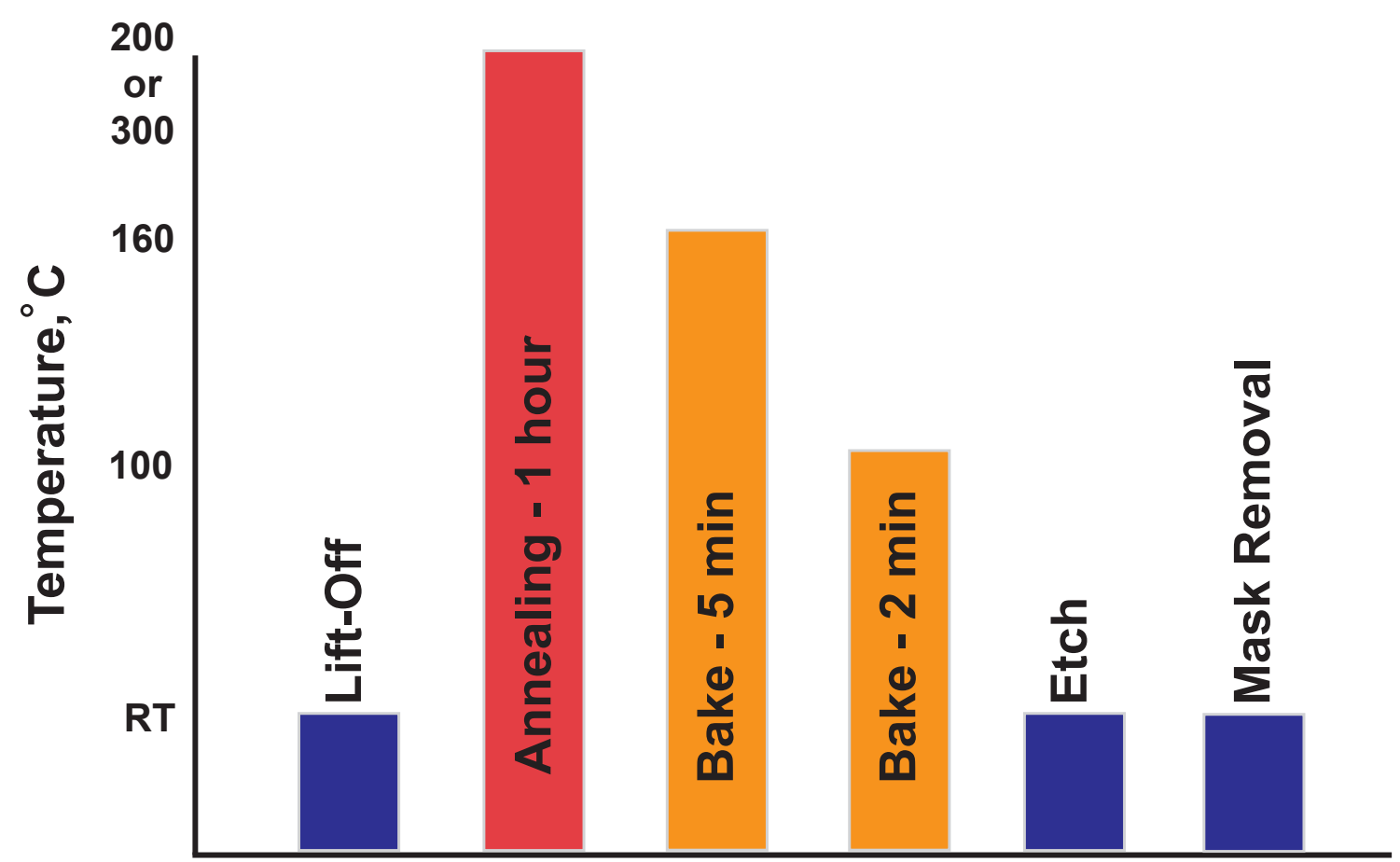

\section{Process Timeline Post Deposition}

Figure 6.6: Timeline of thermal loads post deposition

defined as:

$$
\varepsilon_{t}=\Delta \alpha \cdot \Delta T
$$

where $\Delta \alpha$ is the difference between the coefficients of thermal expansion of the substrate and the film and $\Delta T$ is the difference between the maximum temperature and the deposition temperature, $T-T_{d}$.

In addition to thermal mismatch strain, there are intrinsic strains that may evolve during fabrication that are not linked to thermal mismatch[54]. The difference between expected thermal strains and experimental residual strains may be a representation of the intrinsic strain, $\varepsilon_{i}$, generated during fabrication. Here we simply compare between expected thermal and experimental strains. It proves useful for further discussion to define the final residual strain in the beam as:

$$
\varepsilon_{r}=\varepsilon_{t}+\varepsilon_{i}
$$



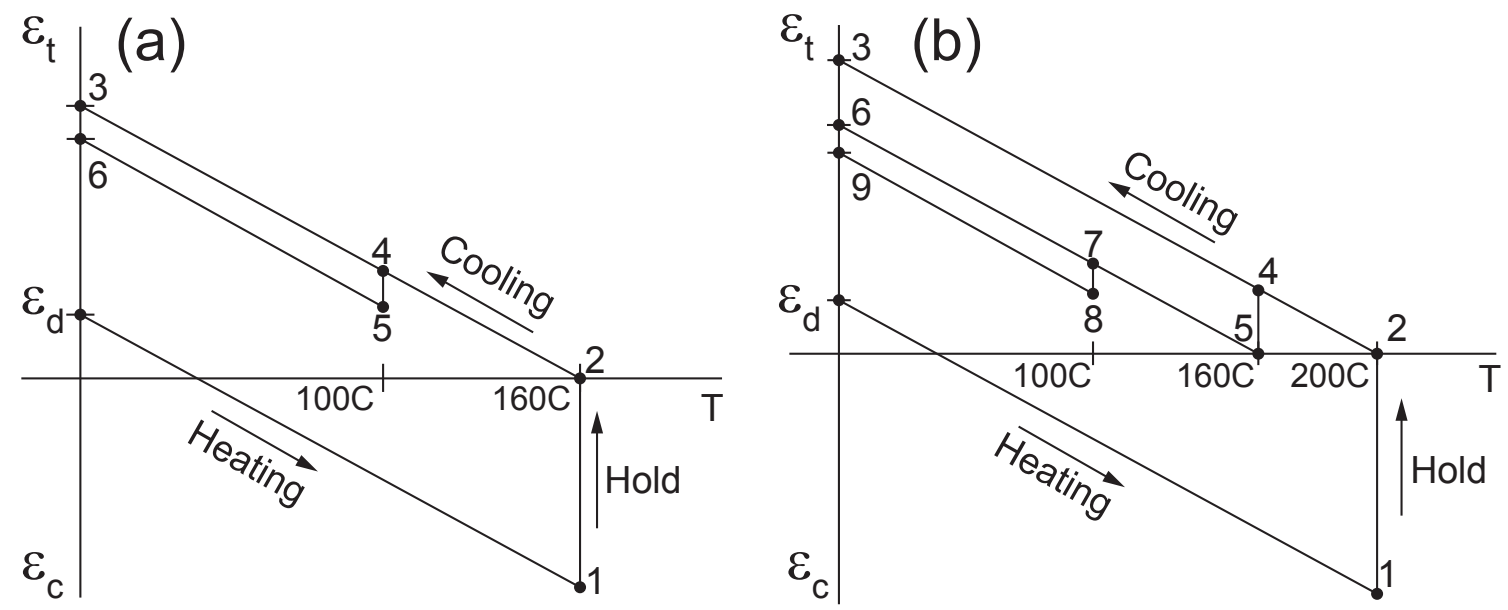

Figure 6.7: Evolution of strain as a function of the temperature seen during fabrication and annealing for the as-fabricated (a) and $200^{\circ} \mathrm{C}$ annealed beams (b). Annealing occurs before the mask is deposited for the etch procedure which influences the strain state in the beam for the $200^{\circ} \mathrm{C}$ annealed beams.

An examination of the thermal processing during fabrication and annealing leads to a better understanding of these strains and how they may vary from their expected strain state.

During the fabrication process all the beams experience $160^{\circ} \mathrm{C}$ for five minutes followed by $100^{\circ} \mathrm{C}$ for two minutes as a part of the mask deposition process. The history of thermal loads seen by beams is shown in figure 6.6. It should be noted that annealing occurs before the mask deposition procedure. Figure 6.7 shows the assumed evolution of strain in the beam as a function of temperature for the as-fabricated (a) and $200^{\circ} \mathrm{C}$ annealed beams (b). The cycle from $1 \rightarrow 3$ in figure 6.7 a represents the five minute bake at $160^{\circ} \mathrm{C}$ during fabrication. The loop from $4 \rightarrow 6$ is the two minute bake at $100^{\circ} \mathrm{C}$. Looking at the results in figure 6.5, it appears the residual strain is primarily from the five minute hold at $160^{\circ} \mathrm{C}$. The $4 \rightarrow 6$ cycle is included as there may be some amount of strain relaxation during the $100^{\circ} \mathrm{C}$ bake. The lower strain in the $42 \mathrm{~nm}$ film suggests there may be some strain relaxation while the $377 \mathrm{~nm}$ thick film appears to have additional intrinsic strain present that cannot be accounted for from thermal mismatch strains. 

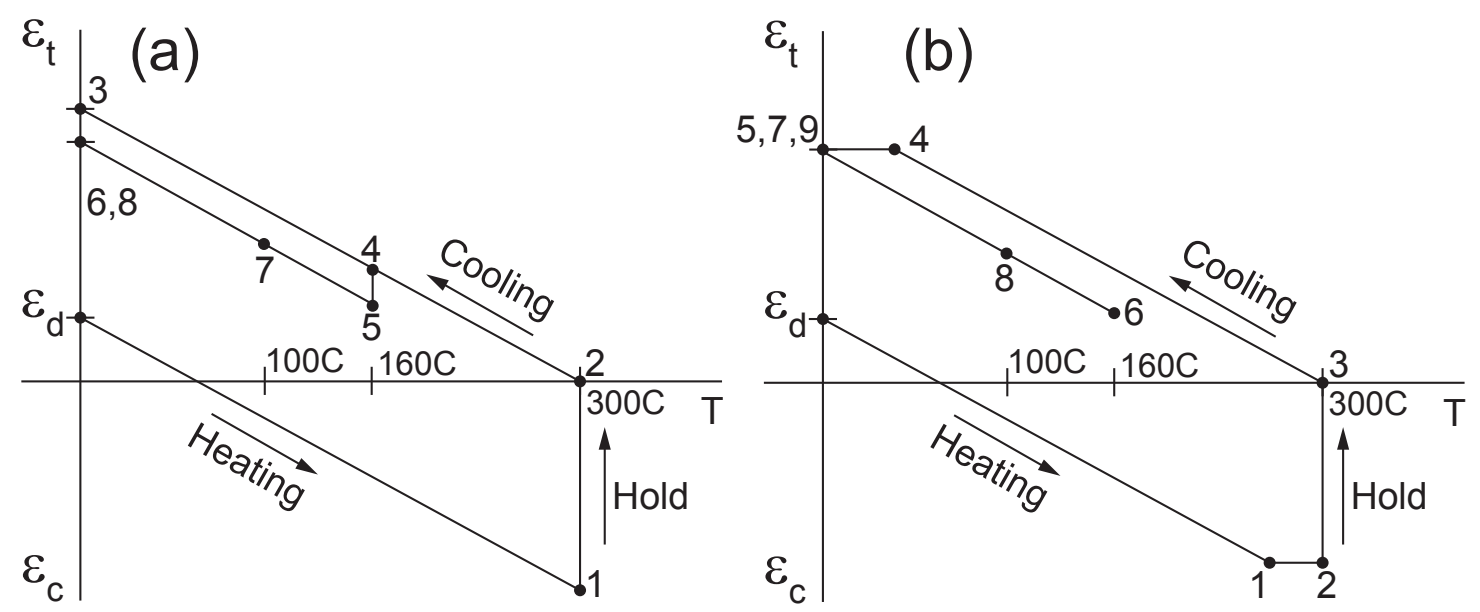

Figure 6.8: Evolution of strain as a function of the temperature seen during fabrication and annealing for the $300^{\circ} \mathrm{C}$ case where the beam is assumed to be unyielded (a) and yielded (b). Relaxation during the fabrication bakes is assumed to be neglible.

Figure 6.7p shows the evolution of strain over the temperature cycling for the $200^{\circ} \mathrm{C}$ annealed beams. The anneal is cycle takes place from $1 \rightarrow 3$. Heating the film to $160^{\circ} \mathrm{C}$ for the PR bake (4) and then holding for five minutes (5) appears to fully relax the strain in the film. At this point the strain-temperature curve becomes identical to that of the as-fabricated film. The results for the $200^{\circ} \mathrm{C}$ anneal in figure 6.5 show nearly identical behavior to the as-fabricated state.

Figure 6.8 1 shows a possible representation of the strain-temperature profile for the $300^{\circ} \mathrm{C}$ annealed case. The assumption in this case is that strain relaxation is either absent or minimal and restricted to the $160^{\circ} \mathrm{C}$ bake. Alternatively, there might be a bit of relaxation from the bake which will not be evident if there is an equal amount of intrinsic stress in the film. Figure $6.8 \mathrm{~b}$ shows the case where the film yields in compression during heating $(1 \rightarrow 2)$ then yields in tension upon cooling $(3 \rightarrow 5)$. Again there is assumed to be no strain relaxation during the fabrication bake cycles. The assumption is that this is the strain state present in the $377 \mathrm{~nm} 300^{\circ} \mathrm{C}$ annealed case and that the reduced residual strain relative to the thinner films is from yielding and not strain relaxation. 


\subsubsection{Modulus}

Much work has been performed to determine the effect of film thickness and grain size on the elastic modulus of thin films[12, 55-60]. Lower modulus due to decreased film thickness is most commonly attributed to the increased volume percentage of softer GBs to the overall makeup of the film[55]. The effects of free surfaces present a higher number of compliant grains and surface atoms with respect to the total volume of very thin films and has been shown via MD to have a significant influence on the elastic modulus of thin films [60]. While there are a host of studies experimentally examining the links between microstructure and mechanical behavior of nickel films [61-66], the large majority have focused on thicker films, primarily fabricated via electrodeposition, and few have investigated the effects of temperature on mechanical properties.

The modulus results, seen in figure 6.10, presents a few interesting and not entirely expected results. The three thicker films appear to have a steady increase in modulus with increasing annealing temperature, plateauing around $200 \mathrm{GPa}$ which is close to the Young's modulus for bulk nickel (205 GPa). The reduced modulus in all cases is generally attributed to the higher percentage of softer grain boundary material present because of the small grain size. This mechanism accurately describes the observed modulus in all cases except the $42 \mathrm{~nm}$ as-fabricated and $200^{\circ} \mathrm{C}$ annealed case where there appear to be additional softening mechanisms in play.

The volume percentage of grain boundary material has been described elsewhere[55] and is represented by:

$$
V_{g b}=1-\left(\frac{d-\Delta_{g}}{d}\right)^{3}
$$

where $V_{g b}$ is the total volume of grain boundary material, $\mathrm{d}$ is the grain diameter and $\Delta_{g}$ is the grain boundary thickness. Figure 6.9 a shows the volume fraction of grain boundary material as a function of grain diameter and grain boundary thickness of $\Delta_{g}=0.5,1.0$ and $1.5 \mathrm{~nm}$. The volume fraction of grain boundaries becomes less than $5 \%$ of the total 

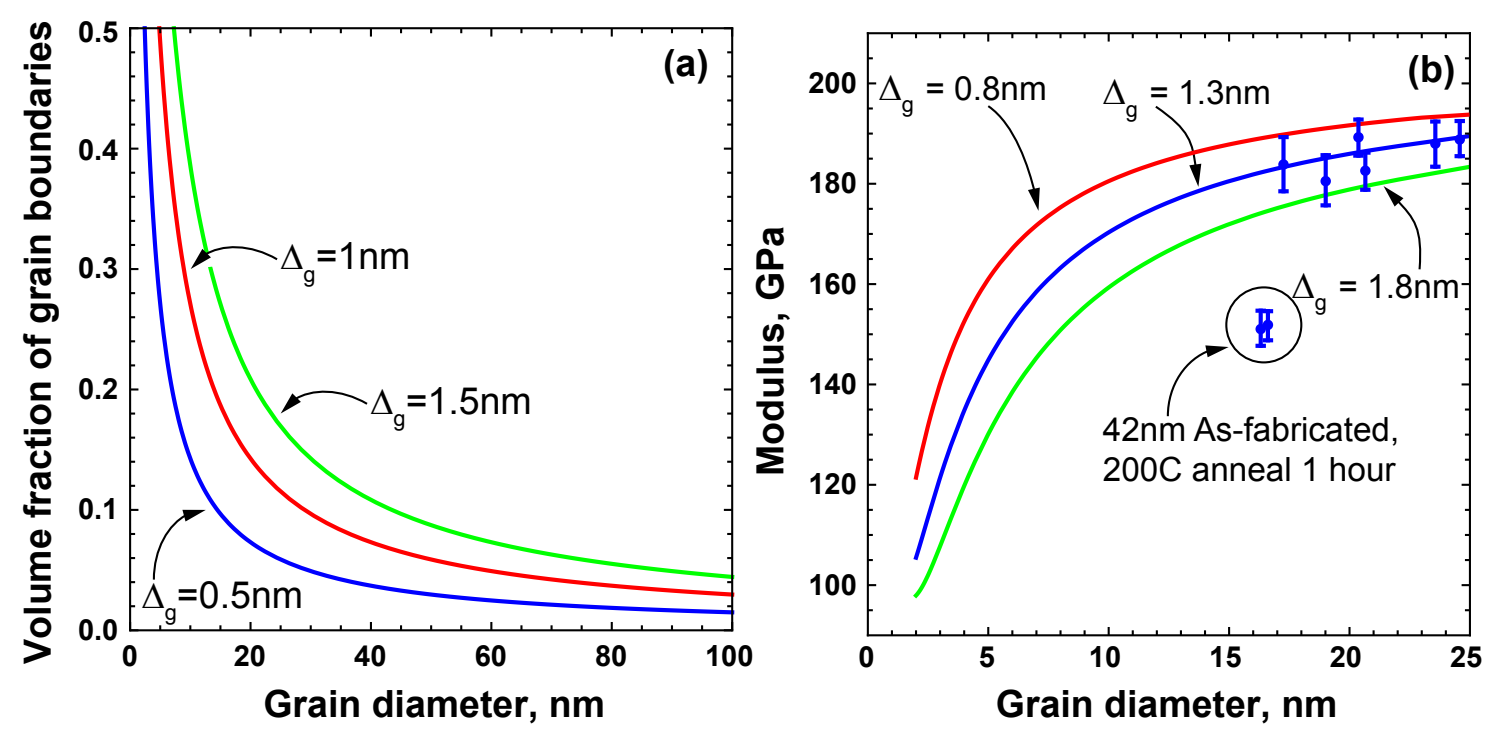

Figure 6.9: Volume fraction of grain boundary material as a function of grain size for three different grain boundary thicknesses (a). Theoretical and experimental modulus as a function of grain diameter. Modulus of the intergranular material and grain boundary material were taken as $205 \mathrm{GPa}$ and 100GPa and the grain boundary thickness appears to vary between 0.8 and $1.8 \mathrm{~nm}(\mathrm{~b})$.

material for all grain boundary thicknesses. Molecular dynamics simulations have recently suggested the modulus of nickel grain boundary material, $E_{g b}$, is around $100 \mathrm{GPa}[60]$. We will use this value in our analyses and assume the intergranular material, $E_{i g}$, to have a modulus of $205 \mathrm{GPa}$. The composite modulus will be defined as:

$$
E_{c}=\left(V_{g b} \cdot E_{g b}+\left(1-V_{g b}\right) \cdot E_{i g}\right) .
$$

Figure 6.9b shows the expected modulus using the values above and grain boundary thickness of $0.8,1.3$ and $1.8 \mathrm{~nm}$. The modulus is within three percent of the bulk value by the time the grain size reaches 50nm. This coincides with the SEM observations for all $300^{\circ} \mathrm{C}$ annealed films as it appears the grain size is larger than approximately 100nm. The modulus for the $300^{\circ} \mathrm{C}$ anneal is around $200 \mathrm{GPa}$ for all thicknesses which corresponds well with the proposed model. 


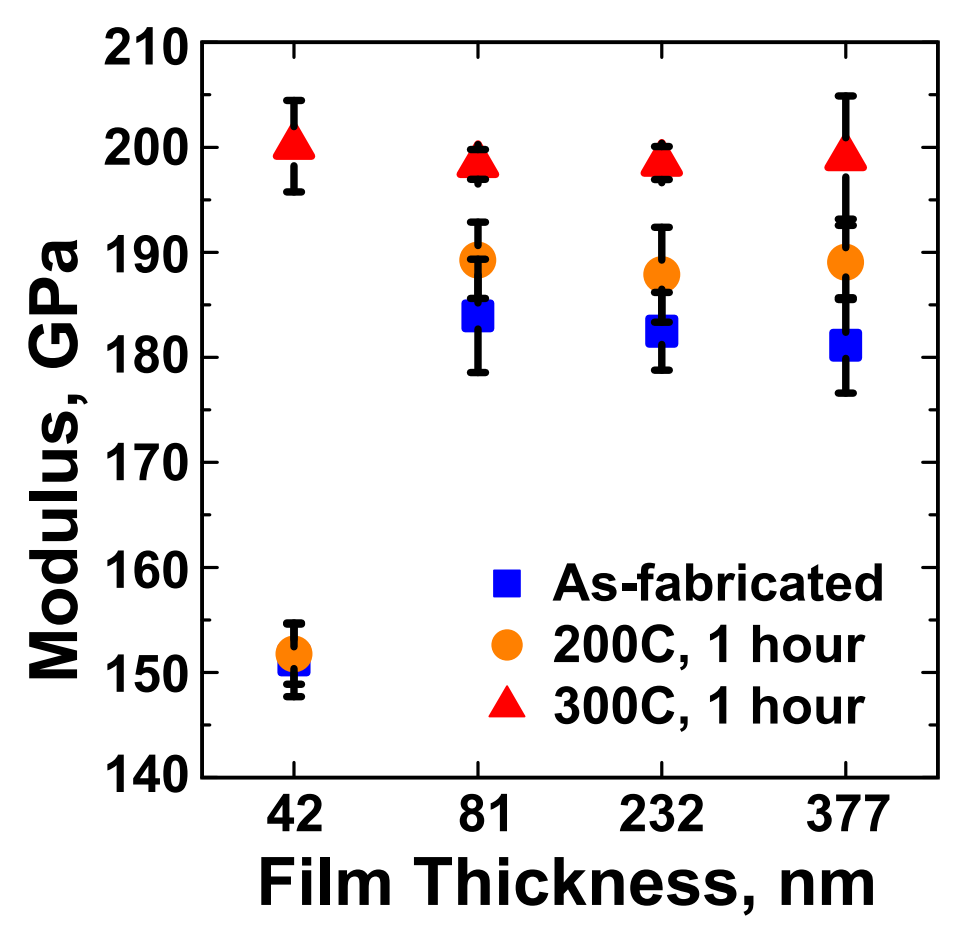

Figure 6.10: Modulus increase with annealing condition which is likely linked to grain size coarsening. The as-fabricated and $200^{\circ} \mathrm{C}$ annealed $42 \mathrm{~nm}$ thick films exhibit $\approx 20 \%$ lower modulus than the thicker films.

The 42nm film shows a significantly lower modulus for both the as-fabricated and $200^{\circ} \mathrm{C}$ anneal condition than the thicker films with the same approximate grain sizes. Recent studies have suggested reduction in modulus for thin films may be linked to surface effects lowering the modulus of surface atoms or variations in surface stress [25, 27, 59, 60, 67]. The pronounced reduction of modulus suggests thickness plays a significant role in the modulus decrease, however, the absence of any reduction in modulus for the $300^{\circ} \mathrm{C}$ case seems to contradict this idea. It appears the $300^{\circ} \mathrm{C}$ anneal may alter any surface effects present in the $42 \mathrm{~nm}$ thick film, raising the modulus to a value in agreement with the thicker $300^{\circ} \mathrm{C}$ annealed films. Additionally, it is possible the reduced modulus effect in the $42 \mathrm{~nm}$ films is only present when the grain size is on the order of the film thickness, which would explain why the $42 \mathrm{~nm} 300^{\circ} \mathrm{C}$ case does not display this additional reduction in modulus. 


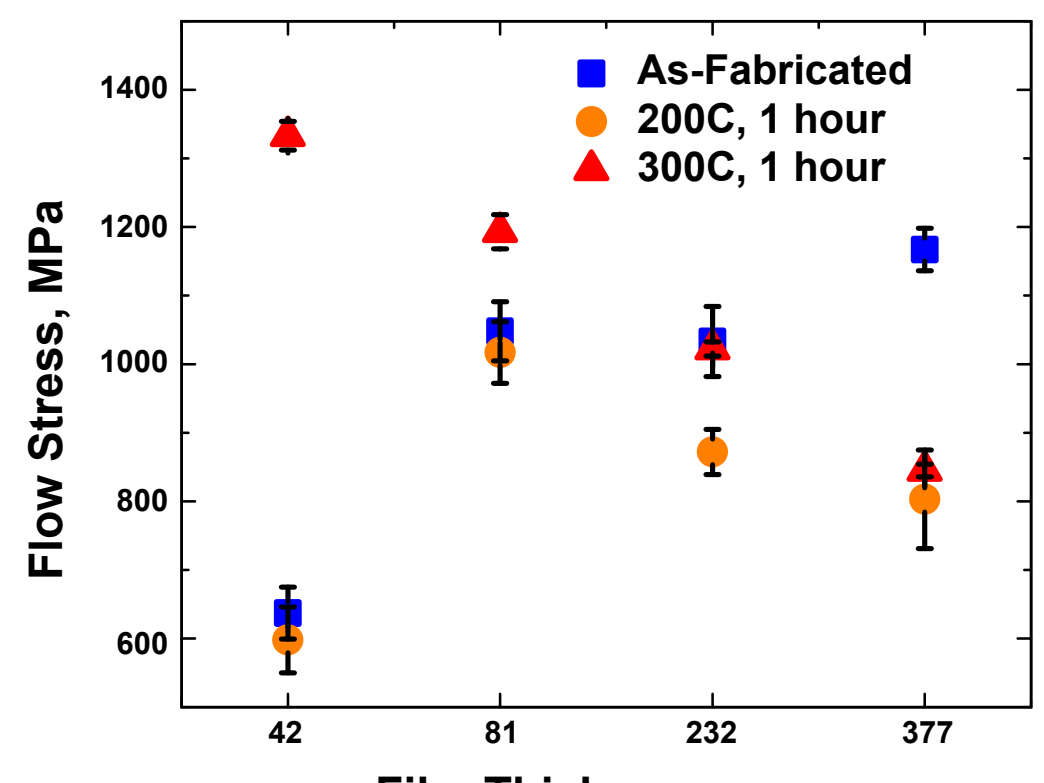

Film Thickness, nm

Figure 6.11: Flow stress as a function of film thickness. Flow stress is defined as the intersection of the stress strain curve with a line of slope equal to the modulus, offset by $0.05 \%$ as seen in figure 6.4 .

\subsubsection{Yield Stress}

The deviation from traditional Hall-Petch behavior in nanocrystalline metals has been studied via molecular dynamics simulations (MD) [42, 68, 69], statistical and continuum mechanics[70, 71] and experiments[61, 62, 72--74]. It is generally accepted that deformation following Hall-Petch behavior is dominated by dislocation plasticity, transitioning to grain boundary (GB) mediated deformations such as GB sliding and GB plasticity as grain sizes decrease below $\sim 20 \mathrm{~nm}[68,71]$.

Initially it is useful to examine the flow stress 1 as a function of film thickness in figure 6.11 to identify trends. One of the more interesting observations is the high initial flow stress in the $300^{\circ} \mathrm{C}$ anneal case for the thinnest film which steadily decreases with increasing film thickness. This increased flow stress for thinner films is likely due to increased difficulty of dislocation motion due to restricted volumes, delaying the onset of plastic

\footnotetext{
${ }^{1}$ The terms yield stress and flow stress will be used interchangeably in this section
} 


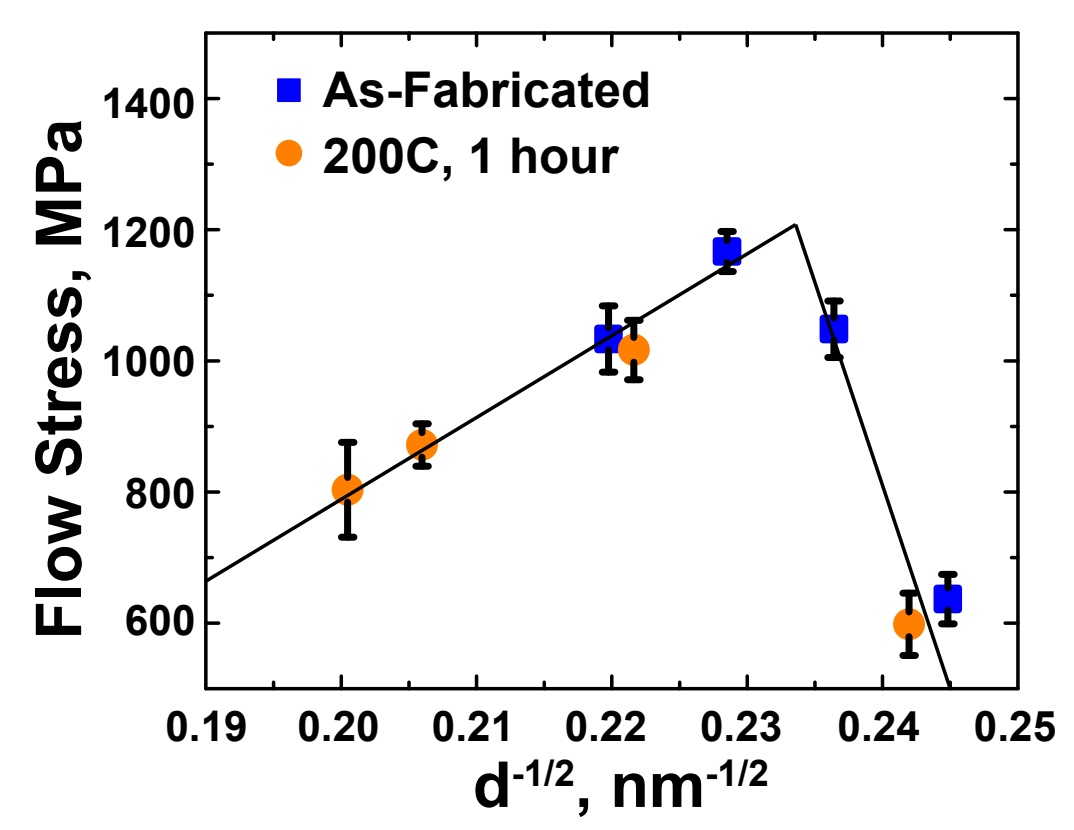

Figure 6.12: Flow stress as a function of square root of inverse grain size. There appears to be a transition from HP to RVHP behavior around a grain size of $18 \mathrm{~nm}$. The lines are added as visual aids.

deformation, and the ability of dislocations to annihilate at the film surface, reducing the accumulation of dislocations in the film interior. The flow and fracture stress of the 42 and $81 \mathrm{~nm}$ thick films appears to be the same as seen in figure 6.13 , which suggests these films exhibit entirely elastic behavior up to fracture. This will be examined in further detail in the discussion of fracture. For the time being we will abandon the $300^{\circ} \mathrm{C}$ films to focus on the as-fabricated and $200^{\circ} \mathrm{C}$ films because the smaller grain sizes tend to display more interesting behavior.

Flow stress as a function of inverse grain size shows the change from traditional HallPetch (HP) behavior, where yield stress increases with decreasing grain size, to reverse Hall-Petch (RVHP), where yield stress decreases with decreasing grain size, as seen in figure 6.12. This effect has been seen a few times experimentally and is largely contributed to deformation mechanisms shifting from dislocation dominated to grain boundary sliding and grain boundary plasticity. The regime where this shift occurs has been postulated, 
and shown in a few cases, to range anywhere from $\sim 5 \mathrm{~nm}$ up to tens of nanometers $[68$ 70, 72, 73]. Here it appears to transition near a grain size of $18 \mathrm{~nm}$ which is consistent with values found in literature. The traditional HP relationship is defined as:

$$
\sigma_{Y S}=\sigma_{Y B}+k \cdot d^{-1 / 2}
$$

where $\sigma_{Y S}$ is the size dependent yield stress, $\sigma_{Y B}$ is the bulk yield stress of nickel and $\mathrm{k}$ is the strengthening coefficient. Fitting the data to the left of the intersection of the HP and RVHP lines in figure 6.12 and taking the bulk yield stress as $\sim 20 \mathrm{MPa}$, the hardening coefficient is about $4500 \frac{M P a}{\sqrt{n m}}$. Previous studies on fine grained nickel have shown the hardening coefficient to be in the range of $\approx 5500 \frac{M P a}{\sqrt{n m}}$ so the value calculated here seems to be reasonable [64, 75].

\subsubsection{Fracture}

The full stress strain curves give insight into fracture stress and fracture strain properties as seen in figure 6.13. The final point on each of the curves is the average of fracture stress and fracture strain from the individual tests. Clearly there is little difference from beam to beam in the general stress strain curve as well as in the fracture properties. Comparing the assumed fracture mode based on the stress strain curve to SEM micrographs we are able to make some general observations about fracture in these films.

Immediately we see that all the $42 \mathrm{~nm}$ thick films exhibit stress strain curves indicative of brittle fracture because of the relative lack of strain hardening upon yielding. The fracture surfaces in figures 6.15 and 6.16 for the as-fabricated and $200^{\circ} \mathrm{C}$ annealed films appear to be nearly identical in nature as are their stress strain curves. The $300^{\circ} \mathrm{C}$ case exhibits a stress strain curve that appears to be almost completely elastic up until fracture. For this film the fracture stress is almost identical to the yield stress from above. The fracture surface in this case from figure 6.17 is different from the other two and looks to be almost 

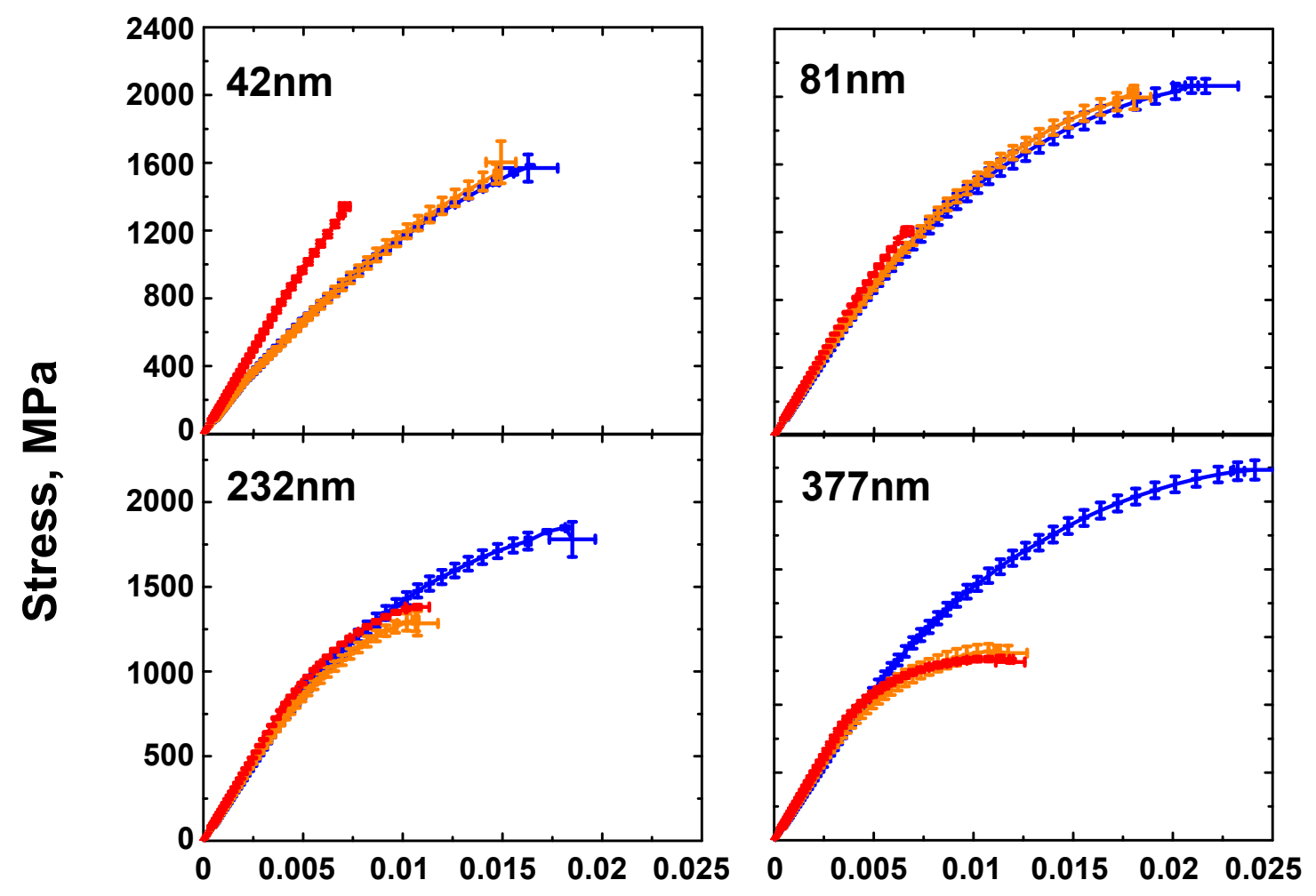

\section{Strain, $\mathrm{mm} / \mathrm{mm}$}

Figure 6.13: Averaged stress strain curves for all tests. Blue lines represent as-fabricated, orange the $200^{\circ} \mathrm{C}$ and red the $300^{\circ} \mathrm{C}$ case. The final data point in each curve is the average fracture stress and fracture strain from the individual tests.

"paper thin" on the top and bottom of the film.

For the $81 \mathrm{~nm}$ thick films the as-fabricated and $200^{\circ} \mathrm{C}$ annealed films again appear to exhibit similar stress strain behavior. An initial look at the as-fabricated fracture surface in figure 6.15 looks like brittle fracture. Upon closer examination in figure 6.14a, there appears to be evidence of plasticity which is consistent with the behavior from the stress strain curve. The $300^{\circ} \mathrm{C}$ shows the brittle fracture expected from the stress strain curve in figures $6.14 \mathrm{~b}$ and 6.17 and again the fracture stress is essentially identical to the yield stress.

The as-fabricated $232 \mathrm{~nm}$ thick film shows similar response to the $81 \mathrm{~nm}$ thick film but 


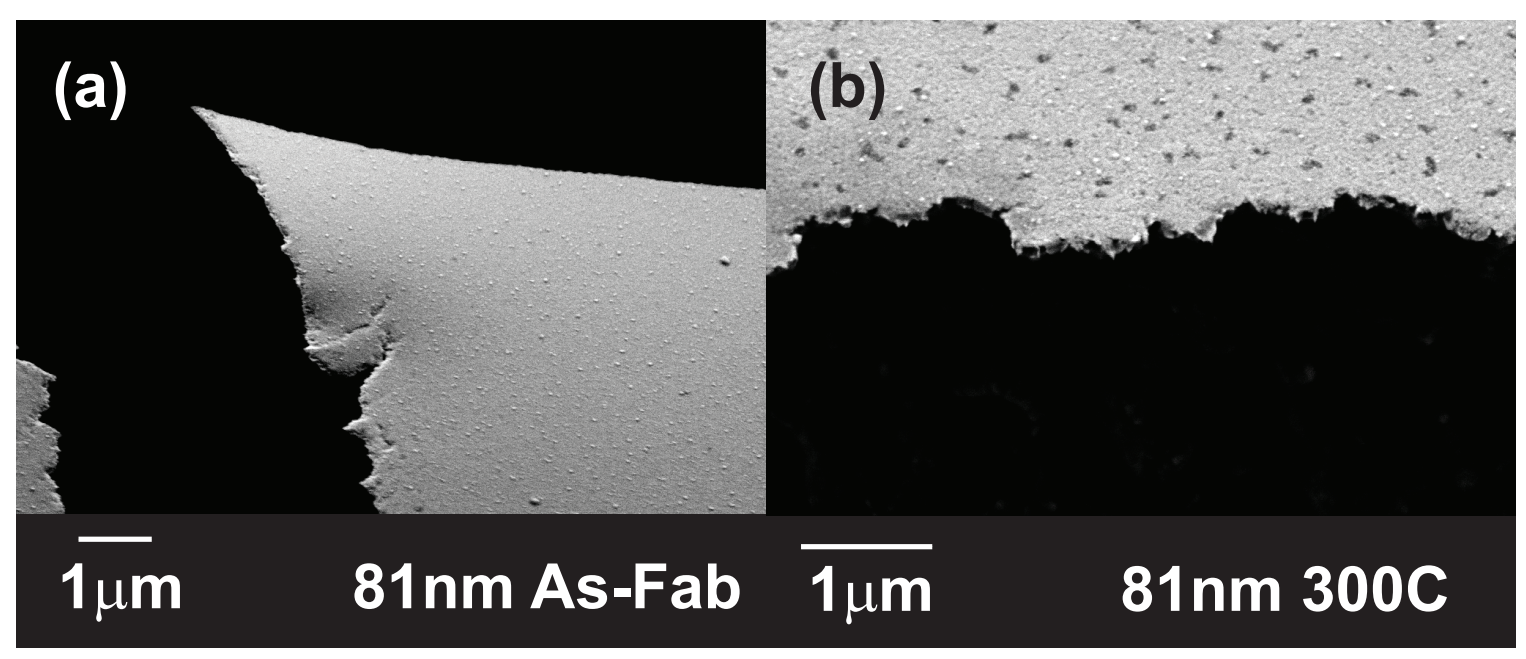

Figure 6.14: SEM of $81 \mathrm{~nm}$ as-fabricated(a) and $300^{\circ} \mathrm{C}(\mathrm{b})$ annealed fracture surface from a tilt angle of 45 degrees. Close examination of the as-fabricated fracture shows evidence of plasticity. The $300^{\circ} \mathrm{C}$ film appears to undergo brittle fracture which is expected from the stress strain curve.

fractures at a lower stress/strain. Interestingly the 200 and $300^{\circ} \mathrm{C}$ annealed films show similar stress-strain behavior whereas they were different in the thinner films. It seems the increase in film thickness for the $200^{\circ} \mathrm{C}$ case has altered the ductility of the $232 \mathrm{~nm}$ film. These observations suggest the thickness of the film has a effect on the fracture properties. The fracture pattern of the $377 \mathrm{~nm}$ film seen in figure 6.17 appears to behave more like the "classic" angled fracture pattern due to shear and less like the brittle fracture seen in the thinner films.

One of the most interesting results is the difference between the stress-strain behavior of the $377 \mathrm{~nm}$ thick films. The as-fabricated film shows twice the ductility and more than twice the fracture stress of the annealed films, which behave almost identically. Again there is a similarity in the yield and fracture behavior of the 200 and $300^{\circ} \mathrm{C}$ films that was not seen in the two thinner films.

Finally it is interesting to examine fracture stress as a function of fracture strains as shown in figure 6.18. Again the absence of scatter should be noted as it speaks to the re- 

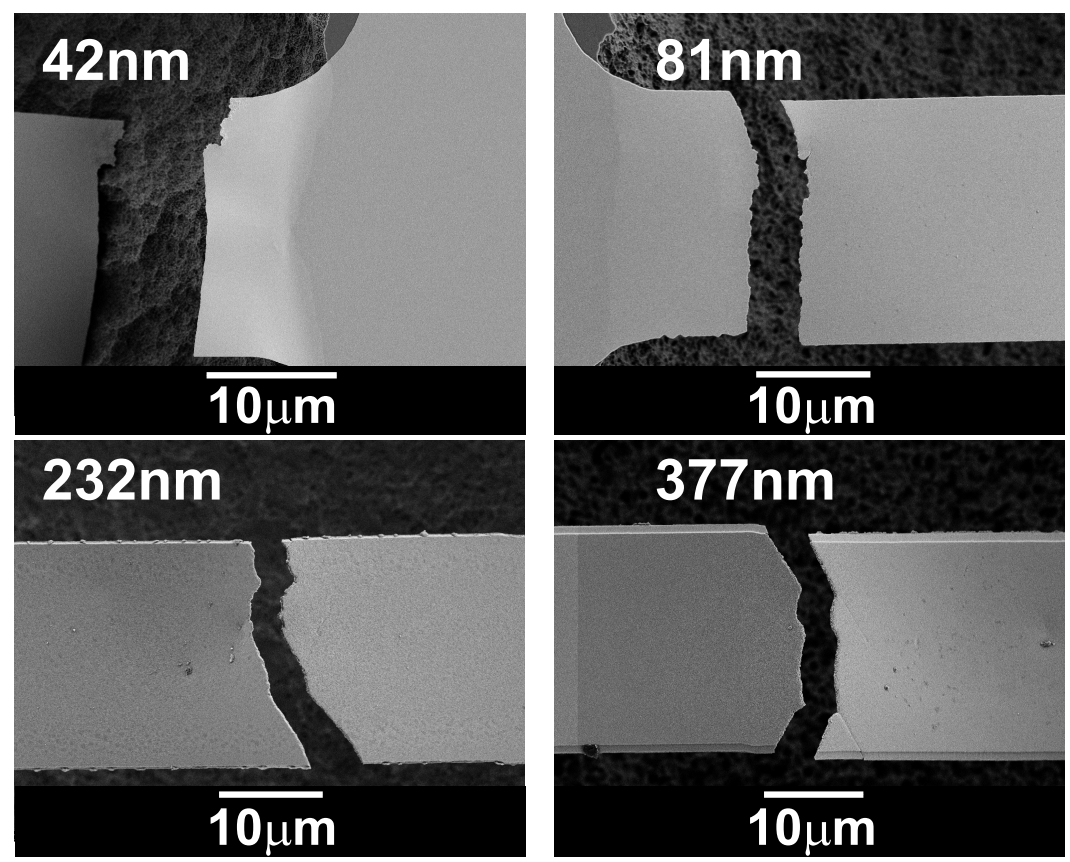

Figure 6.15: As-fabricated fracture surfaces
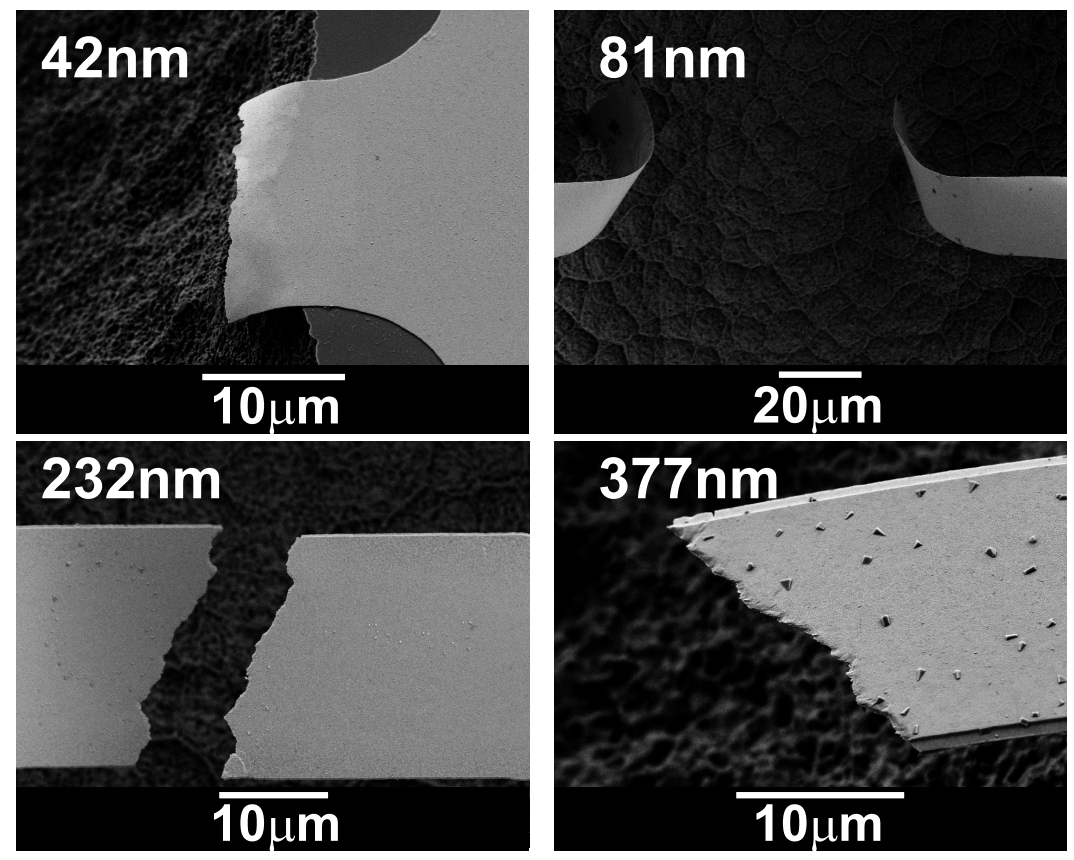

Figure 6.16: $200^{\circ} \mathrm{C}$ fracture surfaces 

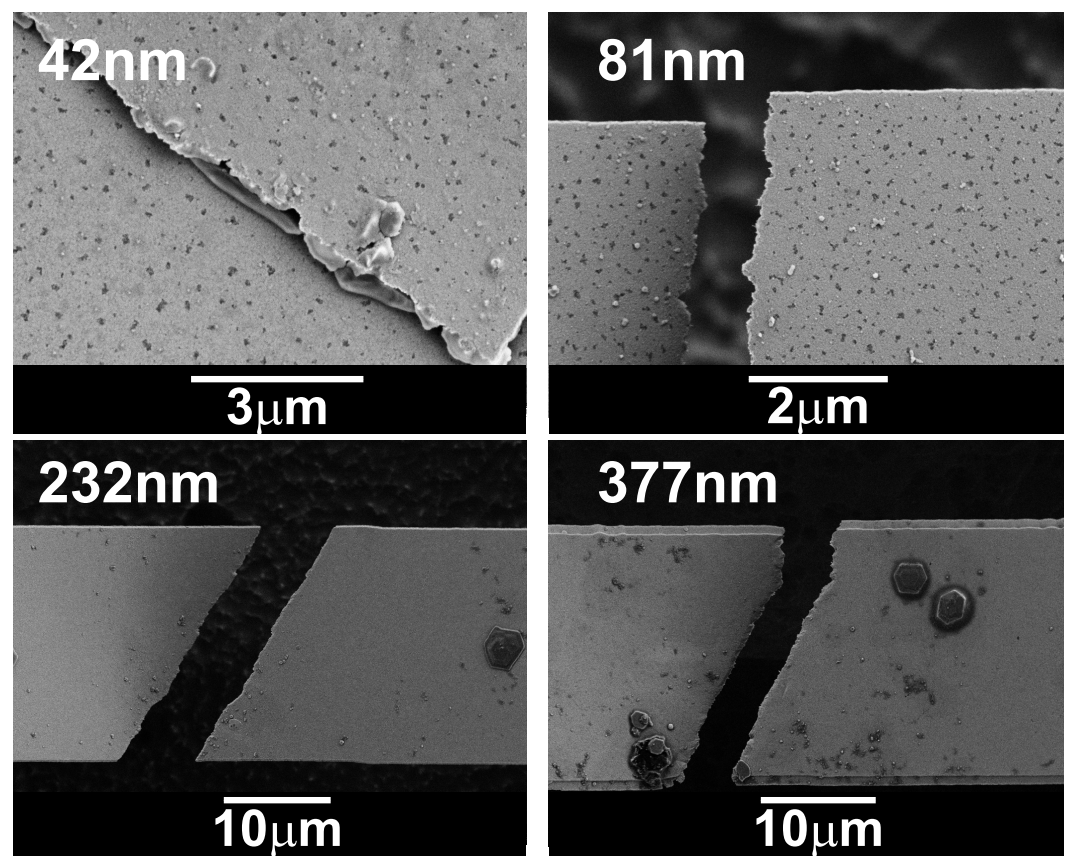

Figure 6.17: $300^{\circ} \mathrm{C}$ fracture surfaces

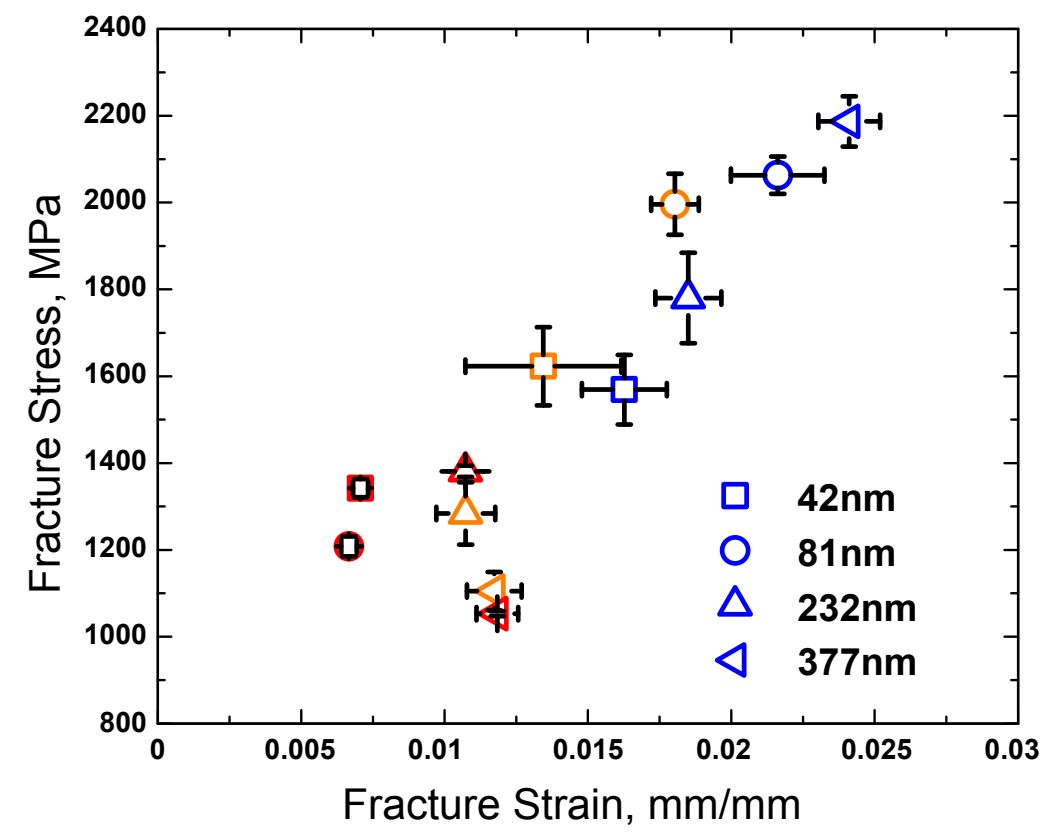

Figure 6.18: Fracture stress as a function of fracture strain for all cases. Following the color convention from previous graphs, blue is as-fabricated, orange $200^{\circ} \mathrm{C}$ anneal and red $300^{\circ} \mathrm{C}$ anneal. Fracture strain is defined as the fracture strain from post-processed (shifted) stress strain curves. 
peatability of tests. Although we do not make any explicit observations about the relationship between fracture stress and fracture strain, the testing and data processing techniques presented here allows for such observations.

\subsection{Conclusion}

Nickel beams were created in their as-fabricated states or annealed at either 200 or $300^{\circ} \mathrm{C}$ to explore the effects of film thickness and microstructural evolution on mechanical properties. A decreased modulus due to small grain size was identified and an unexplained reduction of modulus in the $42 \mathrm{~nm}$ thick film was shown for the as-fabricated and $200^{\circ} \mathrm{C}$ annealed case. The residual strain in beams was shown to generally be attributed to the thermal condition seen by films with explanations presented for lower (relaxation and yield) and higher (intrinsic strain) strains than expected purely from thermal mismatch strains. Yield stress in the as-fabricated and $200^{\circ} \mathrm{C}$ annealed cases showed both traditional HallPetch behavior as well as reverse Hall-Petch behavior at grain sizes smaller than 18nm. A wide range of fracture behavior was seen over the different thickness and annealing conditions. A transition from the $200^{\circ} \mathrm{C}$ annealed beams exhibiting behavior similar to the as-fabricated beams for the thinner films to behavior similar to the $300^{\circ} \mathrm{C}$ beams for thicker films was shown. The study presented displays a wide ranging ability of the testing technique and data interpretation methods used to extract mechanical properties and show links to microstructural and geometrical length scales in a thickness regime where it is difficult to characterize properties with a single testing technique. 


\section{Chapter 7}

\section{Conclusions and Future Work}

This dissertation presents testing techniques, analytical solutions and fabrication methods to extract mechanical properties from freestanding thin film beams. The indentation system has been fully characterized to provide a better understanding of the lower limits of testing with regard to ultra compliant samples. An approximate solution to the response of a point loaded beam under tensile residual stress was presented. A fabrication method to create freestanding beams while minimizing the thermal load on beams during fabrication is shown. Finally, the indentation techniques, analytical solutions and fabrication methods are used to determine the mechanical properties of npAu, $\mathrm{AuAg}$ and nickel beams.

\subsection{Indenter characterization}

The response of the interaction between an imposed harmonic oscillation and the beam have been investigated as a function of the ratio of beam to indenter stiffness and damping, the operating frequency of the oscillatory component and the system parameters (stiffness, damping, natural frequency). This method is used to detect the instant of contact between the indenter and sample and utilizes the under damped resonant characteristics of the indenter assembly. An analysis of the effects of noise in the indentation system was performed and the noise was shown to present as a relatively constant harmonic load of approximately 
$17 \mathrm{nN}$. The system at UVa has an additional noise source near an operating frequency of $\approx$ 140Hz. The raw signals near this operating frequency presents an oscillatory component of $\omega=2 \mathrm{~Hz}$, suggesting there is an environmental vibration coupling with the system near this operating frequency. This is an important analysis which has not previously been presented as it identifies (i) that noise in the system presents as a constant harmonic load, not a constant harmonic oscillation which is commonly quoted in literature, (ii) the approximate value of the noise which can be used to determine the lower threshold of detection as a function of operating frequency for any system and (iii) a system specific noise which identifies specific frequencies that should be avoided during testing of particularly sensitive samples. Additionally a method to test freestanding beams in the membrane regime under constant strain rate was developed and the error between the target strain rate versus that seen experimentally was presented as a function of contact error.

\subsection{Test interpretation}

Analysis of the full non-linear load-deflection response of elastic beams under tensile residual stress allows for extraction of mechanical properties. The full solution as a function of mechanical strain in the beam, $\Lambda$, normalized residual strain, $\bar{\varepsilon}_{R}$ and normalized beam displacement, $\Delta$ was given. Using the approximation $\Lambda=\sqrt{12 c \Delta^{2}+\bar{\varepsilon}_{R}}$, an explicit relationship for load as a function of applied displacement and normalized residual strain is obtained. Adopting $\mathrm{c}=2.12$, the predicted load for a given level of deflection is within $6 \%$ of the exact solution for all displacement ranges. Asymptotic limits were defined corresponding to linear plate, linear pre-stretched membrane and nonlinear membrane solutions. The contribution of bending strains to the total strain in the beam was defined showing that pure stretching is achieved for relatively small applied displacements when residual strain is high, as expected. This result leads to examination of loads and displacements from a geometric approach in order to recreate a traditional stress-strain curve such that elasticplastic properties may be extracted from these tests. 


\subsection{Experimental work}

Work on npAu and AuAg beams showed the variation of mechanical properties as a function of pre and post annealing. Results from a battery of tests on nickel beams of thickness 42, 81, 232 and $377 \mathrm{~nm}$ in either their as-fabricated state or annealed at 200 or $300^{\circ} \mathrm{C}$ were presented. The effects of thermal annealing and temperature seen during fabrication on the residual strain in beams was examined. The variation in modulus as a function of thermal treatment was shown to be linked to grain size of the films in all cases except the $42 \mathrm{~nm}$ as-fabricated and $200^{\circ} \mathrm{C}$ annealed case where there may be a thickness dependence to the modulus. The yield stress in the as-fabricated and $200^{\circ} \mathrm{C}$ appears to follow Hall-Petch behavior up to a grain size of approximately $18 \mathrm{~nm}$ and then exhibits reverse Hall-Petch behavior. The stress-strain curves paired with SEMs of fracture surfaces are used to examine the assumed fracture modes.

\subsection{Future work}

The techniques set forth in this dissertation open a host of doors for future studies. The study presented here on nickel beams would benefit by additional investigation into the microstructure of the films via TEM and/or high-resolution electron backscattered diffraction. An interesting study would be to examine a beam prior to deformation, deform the beam to a strain without fracturing it and then observe changes in microstructure and (if possible) the evolution of dislocation activity and density. This would yield additional insight into the properties presented here and their links to microstructure and small scale deformation mechanisms. One way this might be done would be to back etch the silicon such that TEM could be performed on intact beams without removing them. Additionally, the use of varying strain rates is likely to influence the yield stress and fracture stress/strain characteristics of these films and would be an interesting study. It should be noted that close attention should be paid to the effects of thermal drift on results for slower strain rates.

The use of these methods extends to different film types and film dimensions. It would 
be interesting to create more nickel films with varying thickness close to that of the $42 \mathrm{~nm}$ film in an attempt to elucidate the effects of thickness on the elastic modulus. There has not been an exhaustive study in this area and it would be of great impact. Again the characterization of the microstructure in this case is of pivotal importance. The test procedures laid out here offer a powerful tool in the identification of properties of films with unknown properties. It also allows for the determination of at least modulus and residual strain of nearly any freestanding beam using a commercially available nanoindenter with a point load, which has previously not been possible. 


\section{Bibliography}

[1] E. Seker, J. Gaskins, H. Bart-Smith, J. Zhu, M. Reed, G. Zangari, R. Kelly, and M. Begley, "The effects of annealing prior to dealloying on the mechanical properties of nanoporous gold microbeams," Acta Materialia, vol. 56, no. 3, pp. 324 - 332, 2008.

[2] E. Seker, J. T. Gaskins, H. Bart-Smith, J. Zhu, M. L. Reed, G. Zangari, R. Kelly, and M. R. Begley, "The effects of post-fabrication annealing on the mechanical properties of freestanding nanoporous gold structures," Acta Materialia, vol. 55, no. 14, pp. 4593 $-4602,2007$.

[3] P. W. Lucas, J. T. Gaskins, T. K. Lowrey, M. E. Harrison, H. C. Morrogh-Bernard, S. M. Cheyne, and M. R. Begley, "Evolutionary optimization of material properties of a tropical seed," Journal of the Royal Society Interface, vol. 9, no. 66, pp. 34-42, 2012.

[4] A. Robertson, U. Erb, and G. Palumbo, "Practical applications for electrodeposited nanocrystalline materials," NanoStructured materials, vol. 12, no. 5, pp. 1035-1040, 1999.

[5] G. Palumbo, F. Gonzalez, A. Brennenstuhl, U. Erb, W. Shmayda, and P. Lichtenberger, "In-situ nuclear steam generator repair using electrodeposited nanocrystalline nickel," Nanostructured Materials, vol. 9, no. 1, pp. 737-746, 1997.

[6] T. Deng, M. Prentiss, and G. M. Whitesides, "Fabrication of magnetic microfiltration systems using soft lithography," Applied physics letters, vol. 80, no. 3, pp. 461-463, 2002.

[7] M. Barbic, J. J. Mock, A. P. Gray, and S. Schultz, "Electromagnetic micromotor for microfluidics applications," Applied Physics Letters, vol. 79, no. 9, pp. 1399-1401, 2001.

[8] W. D. Nix, "Mechanical properties of thin films," Metallurgical and Materials Transactions A, vol. 20, pp. 2217-2245, 1989.

[9] S. D. Senturia, Microsystem design. Norwell, MA, USA: Kluwer Academic Publishers, 2001.

[10] S. M. Spearing, "Materials issues in microelectromechanical systems (mems)," Acta Materialia, vol. 48, no. 1, pp. 179 - 196, 2000. 
[11] S. Suresh and B. Freund, Thin Film Materials. Cambridge: Cambridge University Press, 2004.

[12] H. Espinosa, B. Prorok, and M. Fischer, "A methodology for determining mechanical properties of freestanding thin films and mems materials," Journal of the Mechanics and Physics of Solids, vol. 51, no. 1, pp. 47 - 67, 2003.

[13] I. Chasiotis and W. Knauss, "Experimentation at the micron and submicron scale," in Comprehensive Structural Integrity (I. Milne, R. O. Ritchie, , and B. Karihaloo, eds.), pp. 41 - 87, Oxford: Pergamon, 2003.

[14] P. A. Gruber, J. Bohm, F. Onuseit, A. Wanner, R. Spolenak, and E. Arzt, "Size effects on yield strength and strain hardening for ultra-thin cu films with and without passivation: A study by synchrotron and bulge test techniques," Acta Materialia, vol. 56, pp. 2318-2335, 2008.

[15] W. C. Oliver and G. Pharr, "An improved technique for determining hardness and elastic modulus using load and displacement sensing indentation experiments," Journal of Materials Research, vol. 7, pp. 1564-1583, 1992.

[16] T.-Y. Zhang, Y.-J. Su, C.-F. Qian, M.-H. Zhao, and L.-Q. Chen, “Microbridge testing of silicon nitride thin films deposited on silicon wafers," Acta Materialia, vol. 48, no. 11 , pp. $2843-2857,2000$.

[17] K. C. Maner, M. R. Begley, and W. C. Oliver, "Nanomechanical testing of circular freestanding polymer films with sub-micron thickness," Acta Materialia, vol. 52, no. 11, pp. 5451-5460, 2004.

[18] J. Gaskins, N. Barker, and M. Begley, "Comprehensive solutions for the response of freestanding beams with tensile residual stress subject to point-loading," Journal of Applied Mechanics, 2013.

[19] L. Wang and B. Prorok, "Characterization of the strain rate dependent behavior of nanocrystalline gold films," Journal of Materials Research, vol. 23, pp. 55-65, 2007.

[20] E. Herbert, W. Oliver, M. De Boer, and G. Pharr, "Measuring the elastic modulus and residual stress of freestanding thin films using nanoindentation techniques," J. Mater. Res, vol. 24, no. 9, 2009.

[21] M. W. Denhoff, "A measurement of young's modulus and residual stress in mems bridges using a surface profiler," Journal of Micromechanics and Microengineering, vol. 13 , no. 5 , p. $686,2003$.

[22] V. Mulloni, S. Colpo, A. Faes, and B. Margesin, "A simple analytical method for residual stress measurement on suspended mem structures using surface profilometry," Journal of Micromechanics and Microengineering, vol. 23, no. 2, p. 025025 , 2013. 
[23] A. Heidelberg, L. T. Ngo, B. Wu, M. A. Phillips, S. Sharma, T. I. Kamins, J. E. Sader, and J. J. Boland, "A generalized description of the elastic properties of nanowires," Nano Letters, vol. 6, no. 6, pp. 1101-1106, 2006.

[24] L. T. Ngo, D. Almécija, J. E. Sader, B. Daly, N. Petkov, J. D. Holmes, D. Erts, and J. J. Boland, "Ultimate-strength germanium nanowires," Nano letters, vol. 6, no. 12, pp. 2964-2968, 2006.

[25] Z.-J. Wang, C. Liu, Z. Li, and T.-Y. Zhang, "Size-dependent elastic properties of au nanowires under bending and tension-surfaces versus core nonlinearity," Journal of Applied Physics, vol. 108, no. 8, p. 083506, 2010.

[26] D. Zeng and Q. Zheng, "Large deflection theory of nanobeams," Acta Mechanica Solida Sinica, vol. 23, no. 5, pp. 394 - 399, 2010.

[27] E. Celik, I. Guven, and E. Madenci, "Mechanical characterization of nickel nanowires by using a customized atomic force microscope," Nanotechnology, vol. 22, no. 15, p. $155702,2011$.

[28] X. Wang, J. F. Najem, S.-C. Wong, and K. tak Wan, "A nano-cheese-cutter to directly measure interfacial adhesion of freestanding nano-fibers," Journal of Applied Physics, vol. 111, no. 2, p. 024315, 2012.

[29] H. Zhan and Y. Gu, "Modified beam theories for nanowires considering surface/intrinsic effects and axial extension effect," Journal of Applied Physics, vol. 111, April 2012.

[30] R. Djalali, Y.-f. Chen, and H. Matsui, "Au nanowire fabrication from sequenced histidine-rich peptide," Journal of the American Chemical Society, vol. 124, no. 46, pp. 13660-13661, 2002.

[31] Z. Xiao, C. Y. Han, U. Welp, H. Wang, W. Kwok, G. Willing, J. Hiller, R. Cook, D. Miller, and G. Crabtree, "Fabrication of alumina nanotubes and nanowires by etching porous alumina membranes," Nano Letters, vol. 2, no. 11, pp. 1293-1297, 2002.

[32] J. Simmonds, M. Begley, and U. Komaragiri, "The mechanical response of freestanding circular elastic films under point and pressure loads," Journal of applied mechanics, vol. 72, no. 2, pp. 203-212, 2005.

[33] M. R. VanLandingham, "Review of instrumented indentation," tech. rep., DTIC Document, 2003.

[34] M. Doerner and W. Nix, "A method for interpreting the data from depth-sensing indentation instrurnents," J. Mater. Res, vol. 1, no. 4, 1986.

[35] R. Krieg, J. Swearengen, and W. Jones, "A physically based internal variable model for rate dependent plasticity," in Unified Constitutive Equations for Creep and Plasticity, pp. 245-271, Springer, 1987. 
[36] G. Houlsby and A. Puzrin, "Rate-dependent plasticity models derived from potential functions," Journal of Rheology, vol. 46, p. 113, 2002.

[37] I. Chasiotis, C. Bateson, K. Timpano, A. McCarty, N. Barker, and J. Stanec, "Strain rate effects on the mechanical behavior of nanocrystalline au films," Thin Solid Films, vol. 515, no. 6, pp. 3183-3189, 2007.

[38] R. T. Humphrey and A. F. Jankowski, "Strain-rate sensitivity of strength in macro-tomicro-to-nano crystalline nickel," Surface and Coatings Technology, vol. 206, no. 7, pp. 1845-1849, 2011.

[39] J.-F. Huang and I.-W. Sun, "Fabrication and surface functionalization of nanoporous gold by electrochemical alloying/dealloying of au-zn in an ionic liquid, and the selfassembly of 1-cysteine monolayers," Advanced Functional Materials, vol. 15, no. 6, pp. 989-994, 2005.

[40] N. V. Lavrik, C. A. Tipple, M. J. Sepaniak, and P. G. Datskos, "Gold nano-structures for transduction of biomolecular interactions into micrometer scale movements," Biomedical Microdevices, vol. 3, no. 1, pp. 35-44, 2001.

[41] E. Seker, Microfabrication and characterization of nanoporous gold structures. PhD thesis, 2007.

[42] H. Van Swygenhoven and P. Derlet, "Grain-boundary sliding in nanocrystalline fcc metals," Physical review B, vol. 64, no. 22, p. 224105, 2001.

[43] "Microtronics photomask, newtown, pa 18940. www.microtronicsinc.com," tech. rep.

[44] H. Winters and J. Coburn, "The etching of silicon with xef $;$ inf $i \underset{i}{ } 2_{\text {inf }} i$ vapor," $A p$ plied Physics Letters, vol. 34, no. 1, pp. 70-73, 1979.

[45] J. Dagata, D. Squire, C. Dulcey, D. Hsu, and M. Lin, "Chemical processes involved in the etching of silicon by xenon difluoride," Journal of Vacuum Science \& Technology B: Microelectronics and Nanometer Structures, vol. 5, no. 5, pp. 1495-1500, 1987.

[46] K. R. Williams, K. Gupta, and M. Wasilik, "Etch rates for micromachining processing-part ii," Microelectromechanical Systems, Journal of, vol. 12, no. 6, pp. 761-778, 2003.

[47] C. A. Schneider, W. S. Rasband, and K. W. Eliceiri, "Nih image to imagej: 25 years of image analysis," Nat Methods, vol. 9, no. 7, pp. 671-675, 2012.

[48] C. Kril and R. Birringer, "Estimating grain-size distributions in nanocrystalline materials from x-ray diffraction profile analysis," Philosophical Magazine A, vol. 77, no. 3, pp. 621-640, 1998.

[49] H. Natter, M. Schmelzer, and R. Hempelmann, "Nanocrystalline nickel and nickelcopper alloys: Synthesis, characterization, and thermal stability," Journal of Materials research, vol. 13, no. 5, pp. 1186-1197, 1998. 
[50] Y. Wang, S. Cheng, Q. Wei, E. Ma, T. Nieh, and A. Hamza, "Effects of annealing and impurities on tensile properties of electrodeposited nanocrystalline ni," Scripta materialia, vol. 51, no. 11, pp. 1023-1028, 2004.

[51] H. Borchert, E. V. Shevchenko, A. Robert, I. Mekis, A. Kornowski, G. Grübel, and H. Weller, "Determination of nanocrystal sizes: a comparison of tem, saxs, and xrd studies of highly monodisperse copt3 particles," Langmuir, vol. 21, no. 5, pp. 19311936, 2005.

[52] P. Scherrer, "Bestimmung der grösse und der inneren struktur von kolloidteilchen mittels röntgenstrahlen," Nachrichten von der Gesellschaft der Wissenschaften zu Göttingen, Mathematisch-Physikalische Klasse, vol. 1918, pp. 98-100, 1918.

[53] A. Patterson, "The scherrer formula for x-ray particle size determination," Physical review, vol. 56, no. 10, p. 978, 1939.

[54] A. Evans and J. Hutchinson, "The thermomechanical integrity of thin films and multilayers," Acta Metallurgica et Materialia, vol. 43, no. 7, pp. 2507-2530, 1995.

[55] G. Palumbo, S. Thorpe, and K. Aust, "On the contribution of triple junctions to the structure and properties of nanocrystalline materials," Scr. Metall. Mater., vol. 24, no. 7, pp. 1347-1350, 1990.

[56] H. Van Swygenhoven, M. Spaczer, and A. Caro, "Microscopic description of plasticity in computer generated metallic nanophase samples: a comparison between cu and ni," Acta Materialia, vol. 47, no. 10, pp. 3117-3126, 1999.

[57] H. S. Kim and M. B. Bush, "The effects of grain size and porosity on the elastic modulus of nanocrystalline materials," Nanostructured materials, vol. 11, no. 3, pp. 361367, 1999.

[58] M. Haque and M. Saif, "Deformation mechanisms in free-standing nanoscale thin films: A quantitative in situ transmission electron microscope study," Proceedings of the National Academy of Sciences of the United States of America, vol. 101, no. 17, pp. 6335-6340, 2004.

[59] G. Guisbiers, E. Herth, L. Buchaillot, and T. Pardoen, "Fracture toughness, hardness, and youngs modulus of tantalum nanocrystalline films," Applied Physics Letters, vol. 97, no. 14, pp. 143115-143115, 2010.

[60] J. Lian, S.-W. Lee, L. Valdevit, M. I. Baskes, and J. R. Greer, "Emergence of filmthickness- and grain-size-dependent elastic properties in nanocrystalline thin films," Scripta Materialia, vol. 68, no. 5, pp. 261 - 264, 2013.

[61] N. Wang, Z. Wang, K. Aust, and U. Erb, "Room temperature creep behavior of nanocrystalline nickel produced by an electrodeposition technique," Materials Science and Engineering: A, vol. 237, no. 2, pp. 150-158, 1997. 
[62] A. Godon, J. Creus, S. Cohendoz, E. Conforto, X. Feaugas, P. Girault, and C. Savall, "Effects of grain orientation on the hall-petch relationship in electrodeposited nickel with nanocrystalline grains," Scripta Materialia, vol. 62, no. 6, pp. 403-406, 2010.

[63] A. El-Sherik, U. Erb, G. Palumbo, and K. Aust, "Deviations from hall-petch behaviour in as-prepared nanocrystalline nickel," Scripta Metallurgica et Materialia(USA), vol. 27, no. 9, pp. 1185-1188, 1992.

[64] C. Xiao, R. Mirshams, S. Whang, and W. Yin, "Tensile behavior and fracture in nickel and carbon doped nanocrystalline nickel," Materials Science and Engineering: A, vol. 301, no. 1, pp. 35-43, 2001.

[65] W. Yin, S. Whang, R. Mirshams, and C. Xiao, "Creep behavior of nanocrystalline nickel at 290 and 373 k," Materials Science and Engineering: A, vol. 301, no. 1, pp. 18-22, 2001.

[66] F. Dalla Torre, H. Van Swygenhoven, and M. Victoria, "Nanocrystalline electrodeposited ni: microstructure and tensile properties," Acta Materialia, vol. 50, no. 15, pp. 3957-3970, 2002.

[67] R. Dingreville, J. Qu, and M. Cherkaoui, "Surface free energy and its effect on the elastic behavior of nano-sized particles, wires and films," Journal of the Mechanics and Physics of Solids, vol. 53, no. 8, pp. 1827-1854, 2005.

[68] D. Wolf, V. Yamakov, S. Phillpot, A. Mukherjee, and H. Gleiter, "Deformation of nanocrystalline materials by molecular-dynamics simulation: relationship to experiments?," Acta Materialia, vol. 53, no. 1, pp. 1-40, 2005.

[69] M. Koslowski, D. W. Lee, and L. Lei, "Role of grain boundary energetics on the maximum strength of nanocrystalline nickel," Journal of the Mechanics and Physics of Solids, vol. 59, no. 7, pp. 1427-1436, 2011.

[70] C. Carlton and P. Ferreira, "What is behind the inverse hall-petch effect in nanocrystalline materials?," Acta materialia, vol. 55, no. 11, pp. 3749-3756, 2007.

[71] Y. Wei and L. Anand, "Grain-boundary sliding and separation in polycrystalline metals: application to nanocrystalline fcc metals," Journal of the Mechanics and Physics of Solids, vol. 52, no. 11, pp. 2587-2616, 2004.

[72] C. Schuh, T. Nieh, and T. Yamasaki, "Hall-petch breakdown manifested in abrasive wear resistance of nanocrystalline nickel," Scripta Materialia, vol. 46, no. 10, pp. 735-740, 2002.

[73] H. Conrad and J. Narayan, "Mechanism for grain size softening in nanocrystalline zn," Applied physics letters, vol. 81, no. 12, pp. 2241-2243, 2002.

[74] M. Haque and M. A Saif, "Mechanical behavior of 30-50 nm thick aluminum films under uniaxial tension," Scripta Materialia, vol. 47, no. 12, pp. 863-867, 2002. 
[75] F. Ebrahimi, G. Bourne, M. S. Kelly, and T. Matthews, "Mechanical properties of nanocrystalline nickel produced by electrodeposition," Nanostructured Materials, vol. 11, no. 3, pp. 343-350, 1999.

[76] H. W. Hilhorst, "Definitions and hypotheses of seed dormancy," Annual Plant Reviews Volume 27: Seed Development, Dormancy and Germination, pp. 50-71, 2007.

[77] F. Dieter and F. Bouman, "The seed: Structure and function," Seed Development Germination, vol. 41, p. 1, 1995.

[78] J. Richardson, L. Chatrou, J. Mols, R. Erkens, and M. Pirie, "Historical biogeography of two cosmopolitan families of flowering plants: Annonaceae and rhamnaceae," Philosophical Transactions of the Royal Society of London. Series B: Biological Sciences, vol. 359, no. 1450, pp. 1495-1508, 2004.

[79] W. Tze, S. Wang, T. Rials, G. Pharr, and S. Kelly, "Nanoindentation of wood cell walls: continuous stiffness and hardness measurements.," Composites A, vol. 38, pp. 945-953, 2007.

[80] A. D. Tomos and R. A. Leigh, "The pressure probe: a versatile tool in plant cell physiology," Annual review of plant biology, vol. 50, no. 1, pp. 447-472, 1999.

[81] C. Wheater and M. Evans, "The mandibular forces and pressures of some predacious coleoptera," Journal of insect physiology, vol. 35, no. 11, pp. 815-820, 1989.

[82] S. Lee, N. Yaakob, K. S. Boon, and L. S. L. Chua, "The role of selected animals in pollinatino and dispersal of trees in the forest: implications for conservation and management," J. Trop. For. Sci., vol. 14, pp. 234-263, 2002.

[83] M. E. Harrison, Orang-utan feeding behaviour in Sabangau, Central Kalimantan. PhD thesis, 2009.

[84] E. R. Vogel, J. T. van Woerden, P. W. Lucas, S. S. Utami Atmoko, C. P. van Schaik, and N. J. Dominy, "Functional ecology and evolution of hominoid molar enamel thickness: $i i_{i}$ pan troglodytes schweinfurthii $i_{i}$ and $i_{i} i_{i}$ pongo pygmaeus wurmbii $/$ /i,$"$ Journal of Human Evolution, vol. 55, no. 1, pp. 60-74, 2008.

[85] P. W. Lucas, C. R. Peters, and S. R. Arrandale, "Seed-breaking forces exerted by orang-utans with their teeth in captivity and a new technique for estimating forces produced in the wild," American Journal of Physical Anthropology, vol. 94, no. 3, pp. 365-378, 1994. 


\section{Chapter 8}

\section{Appendix}

\subsection{Photolithography Masks}

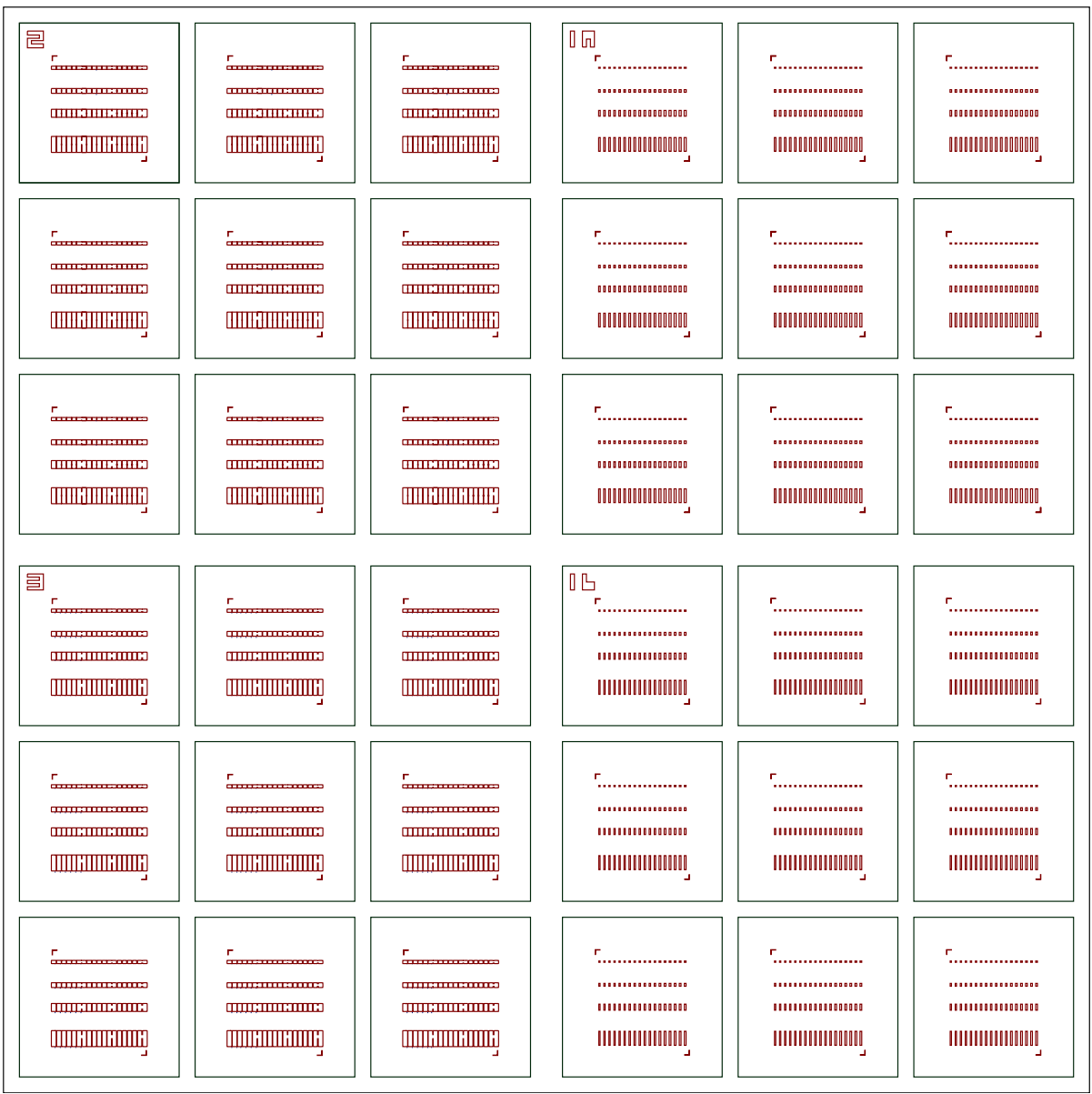

Figure 8.1: Beam masks for fabrication. Mask three was used for all beams in chapter 6 . The etch masks (1a and $1 \mathrm{~b}$ ) contain varying difffering sizes of etch rectangles. Mask two was an experimental mask and was unused. 


\title{
8.2 Fabrication procedure for freestanding beams
}

\author{
Substrate
}

1. 2-inch silicon wafer

2. $275 \mu$ m-thick

3. (100) orientation

4. P-type Boron doped

5. Single side polish

Beam patterning and deposition for evaporation and sputter

1. Ethanol, trichloroethylene (TCE), and methanol (ETM) clean

2. Spin LOR10B - $4000 \mathrm{rpm}-30 \mathrm{sec}$

3. Bake $160^{\circ} \mathrm{C}-5 \mathrm{~min}$

4. Spin AZ4210 - $4000 \mathrm{rpm}-30 \mathrm{sec}$

5. Bake $100^{\circ} \mathrm{C}-2$ min

6. Expose $45 \mathrm{sec}-7 \mathrm{~mW} / \mathrm{cm}^{2}$ - Mask 3 - Align bottom flat perpendicular to beams for dicing

7. Develop AZ400K (1:4) - $60 \mathrm{sec}$

8. DI rinse $1 \mathrm{~min}$

9. $\mathrm{N}_{2}$ blow dry

10. Deposit adhesion layer (if necessary)

11. Deposit desired film thickness

12. Lift-off in room temperature (RT) NMP in low ultrasonic agitation $30 \mathrm{~min}$, changing NMP bath half-way through

13. DI rinse $1 \mathrm{~min}$

14. $\mathrm{N}_{2}$ blow dry

15. Measure film thickness with mechanical profilometer

\section{Beam patterning for electroplating}

1. ETM clean

2. Evaporate or sputter $10 \mathrm{~nm}$ Ti and $100 \mathrm{~nm}$ seed layer of whatever material you are plating

3. ETM clean

4. Spin AZ4210 - $4000 \mathrm{rpm}-30 \mathrm{sec}$ (for beams ; $2 \mu \mathrm{m}$ thickness)

5. Bake $100^{\circ} \mathrm{C}-2 \mathrm{~min}$

6. Expose $45 \mathrm{sec}-7 \mathrm{~mW} / \mathrm{cm}^{2}$ - Mask 3 - Align bottom flat perpendicular to beams for dicing

7. Develop AZ400K (1:4) - $60 \mathrm{sec}$

8. Measure resist thickness with mechanical profilometer

9. Electoplate for $5 \mathrm{~min}$

10. Measure PR with mechanical profilometer to get deposition rate

11. Electroplate until desired thickness is achieved

12. Remove PR with acetone 13 . DI rinse $14 . \mathrm{N}_{2}$ blow dry

15. Measure film thickness with mechanical profilometer 


\section{Dicing}

1. Carbon scribe small notches perpendicular to bottom flat along channels left from mask

2. Continue dicing all 9 samples into square samples for uniformity

\section{Etch mask patterning}

1. ETM clean

2. Spin LOR10B - $4000 \mathrm{rpm}-30 \mathrm{sec}$

3. Bake $160^{\circ} \mathrm{C}-5 \mathrm{~min}$

4. Spin AZ4210 - $4000 \mathrm{rpm}-30 \mathrm{sec}$

5. Bake $100^{\circ} \mathrm{C}-2 \mathrm{~min}$

6. Expose $45 \mathrm{sec}-7 \mathrm{~mW} / \mathrm{cm}^{2}$ - Mask $1 \mathrm{~b}$

7. Develop AZ400K (1:4) - $60 \mathrm{sec}$

8. DI rinse $1 \mathrm{~min}$

9. $\mathrm{N}_{2}$ blow dry

10. Remove AZ4210 resist - Acetone bath - 20 min

11. Methanol rinse - $1 \mathrm{~min}$

12. DI rinse $1 \mathrm{~min}$

13. $\mathrm{N}_{2}$ blow dry

\section{Etch Protocols}

1. Cut a square in a piece of tape larger than the patterned beam area but smaller than the sample (i.e. don't mask the beams but don't leave anything unmasked by PR exposed)

2. Vent the Xetch ${ }^{\circledR}$ e1 Series etcher and tape the sample to the platter

3. Pump down the chamber

4. In etch mode, designate three cycles, two minutes per cycle with a gas pressure of two mTorr (note these parameters are dependent upon area of exposed Si being etched)

5. Carefully remove the tape from the platter and examine sample under a microscope. If fully etched remove tape, if not continue etching until completed

\section{LOR10B removal}

1. Place sample in AZ400K for $\sim 1$ hour

2. Remove from AZ400K and soak in methanol bath for $20 \mathrm{~min}$

3. Place sample on clean room cloth to air dry

\subsection{Study on the tropical seed Mezzetia Parviflora}

The tropical seed Mezzettia parviflora is an example of a material systems where mechanical properties are shown to be directly influenced by microstructural length scales. A study on M. parviflora is used to show how its anisotropic mechanical properties and microstructural architecture have optimized to permit germination while protecting against damage mechanisms produced by an array of predators.

Seeds often posses architectures that carefully protect against damage from predators by a protective shell while still allowing for germination[76]. While most angiosperm 

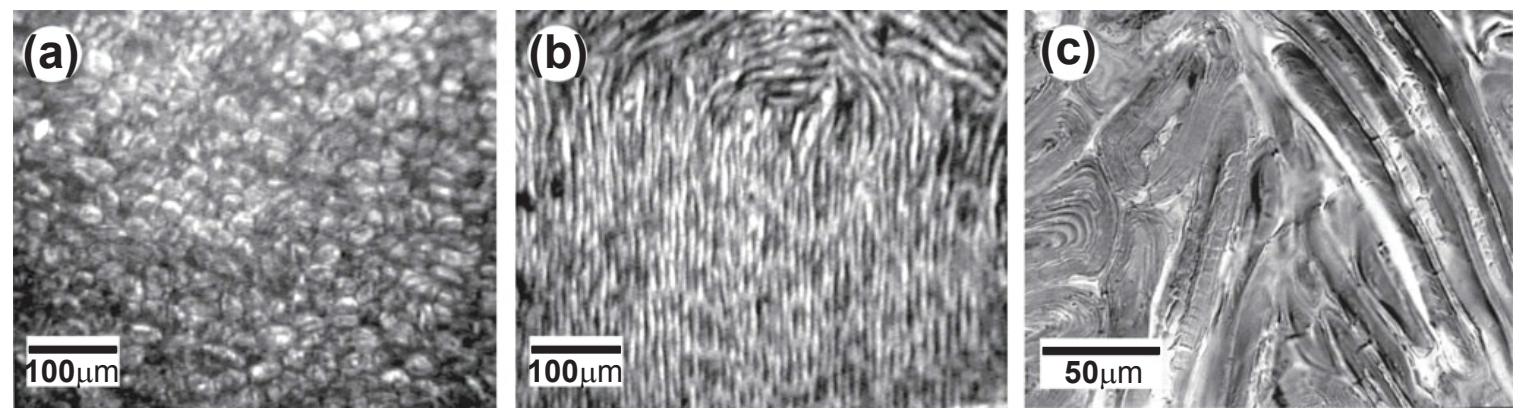

Figure 8.2: Light microscopy of (a) short stubby cells in zone III, (b) parallel fibers of zone I (bottom of image) grading into randomly oriented zone II fibers (top), and (c) short stubby cells of zone III interspersed amongst random fibers of zone II[3].

families have trended toward a simplification of the integument layers from two to one, a few southeast Asian members of the palaeodicot family Annonaceae have evolved a third layer[77, 78]. The evolution of this third layer suggests an increased need for protection of the seed's inner embryo. The large tropical seed M. parviflora is known to have a number of predators including beetles, squirrels and orangutans. A mechanical analysis is used to show how this seed protects itself from predation while still allowing for germination by adaptation of a unique cellular structure. Links are made between the architecture of the shell, mechanical properties and shell failure mechanisms delivered via predators. While the general mechanisms will be addressed briefly with regard to the mechanical properties measured via indentation, more attention will be paid to the methods used to extract the micro-scale properties which contribute to the macro scale failure analysis. The macro scale failure analysis were primarily the work of Peter Lucas and Matthew Begley.

In order to estimate the forces necessary to activate macro scale failure mechanisms, it is necessary to determine properties at the structural level of the seed. First a discussion of the structure of the seed is necessary as spatial variations in shape and orientation will lead to important differences in mechanical properties. Figure 8.2 shows light microscopy of three distinct region that are of importance in this study. Their location on the macro scale nut can be seen in figure 8.5. Zone I consists of parallel fibers at the outer edge of the nut. As these fibers run towards the center of the nut they begin to randomly orient in bundles until the entire fiber matrix is randomly oriented, which is denoted as zone II. The final area of interest is denoted as zone III and is made up of pseudospherical stubby cells which form a $0.5 \mathrm{~mm}$ tall germination band around the seed. This germination band is the area where a crack propogates during germination to allow the seed to open and eject the hypocotyl. A diamond-shaped plug, seen at the bottom of figure 8.5, is positioned at one end of the nut and acts as a source for a crack as its ends which run parallel to the germination band dramatically thin as they run into zone III material.

In order to prepare these samples for indentation testing they were first cut from a section of nut and polished down to create a flat surface. An incremental polishing routine first 


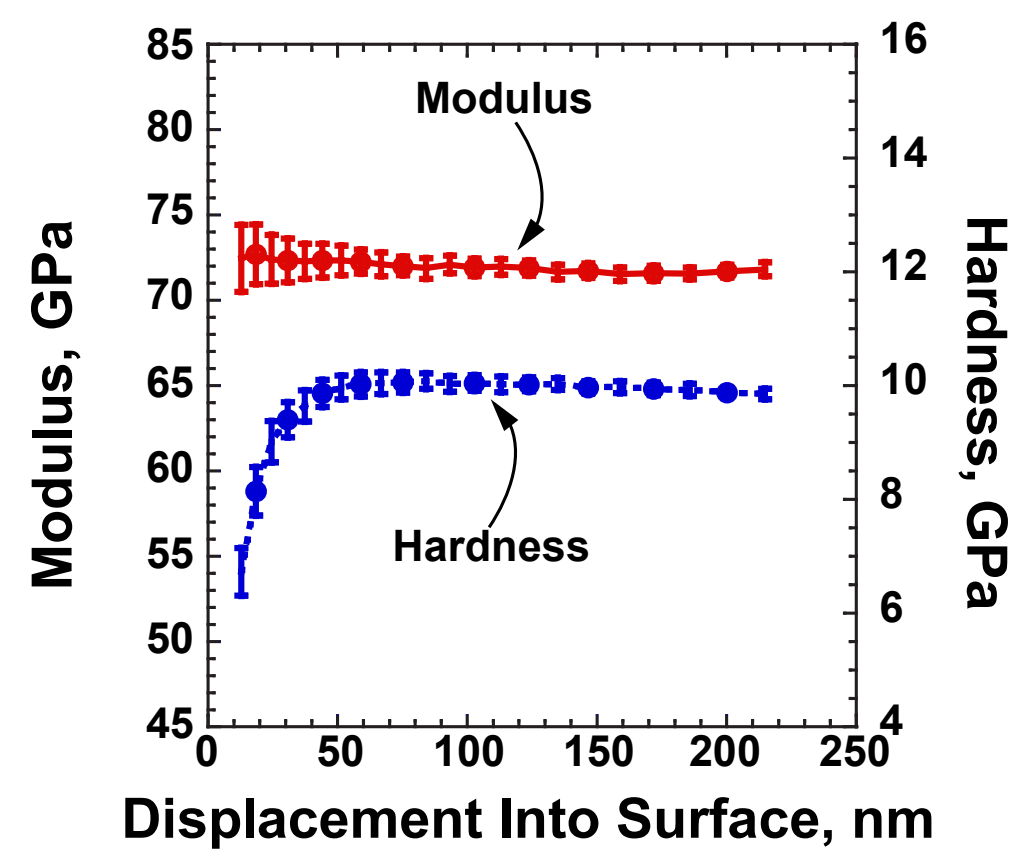

Figure 8.3: Modulus and hardness as a function of indenter displacement. Calibration values are valid for depths greater than $\sim 20 \mathrm{~nm}$.

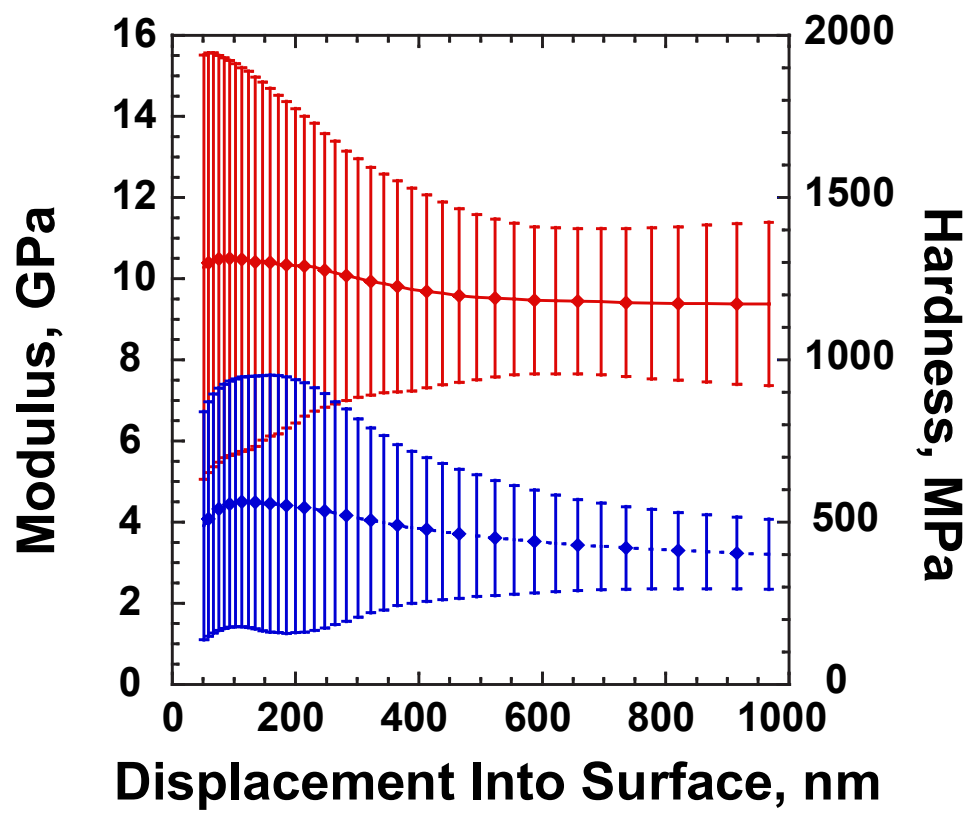

Figure 8.4: Modulus and hardness in the randomly oriented fiber region (zone II). The large scatter at small depths reduces as larger volumes of the material are sampled and the response becomes indicative of the bulk material response. 
Table 8.1: Mechanical properties of M. parviflora for different locations. Mean (s.d.)

\begin{tabular}{ccc}
\hline \hline properties & modulus, E $(\mathrm{GPa})$ & hardness, H $(\mathrm{MPa})$ \\
\hline scale of test $(\mathrm{mm})$ & $<0.01$ & $<0.01$ \\
zone I: parallel fibers & 1 axial $15.7(0.9),{ }^{1}$ parallel $7.2(0.7)$ & $400(110)$ \\
zone II: randomly oriented fibers & $9.4(2.0)$ & $490(110)$ \\
zone III: pseudospherical cells & $10.7(1.1)$ & $490(110)$ \\
\hline \hline
\end{tabular}

using small grit polishing papers with diminishing grit sizes and then finishing them with alumina slurries with decreasing diameter particles, with the smallest particle size being $0.05 \mu \mathrm{m}$ until a glossy finish was present. This glossy finish is analogous to a mirror finish in metals. This polishing process was necessary to ensure a planar surface over which to perform indentation tests.

Nanoindentation tests were performed equipped with a Berkovich diamond tip of calibrated head radius of approximately 100nm, a similar set-up to recent studies on wood[79]. The calibration was performed on fused silica and shown in figure 8.3 . For all tests, modulus and hardness over displacement data were made using the continuous stiffness method to a depth of $1 \mu \mathrm{m}$. A drift correction factor, as determined by a 50s hold step at 10 percent of the maximum load during unloading, was applied to the data to account for thermal drift during the test. Measurements were the average of at least 15 indentations, with 55 tests being completed on the randomly oriented fibers of zone II, as seen in figure 8.4 , to ensure that variations in local material properties did not influence the global average.

Measurements of shell mechanical properties at the micrometer scale are summarized in table 8.1. In general, the nanoindentation tests show large scatter over small depths and asymptote to a value at depths of about $1 \mu \mathrm{m}$, at which depth values in table 8.1 were taken. Loads at this depth varied between 7.5 and $10 \mathrm{mN}$. The shell has a nearly spatially uniform hardness - the highest recorded for nut shells. While the elastic modulus varies more than hardness, the major influence is whether loading on the fibers is axial or transverse. Clearly in zone II, the mean modulus is a statistical average over a range of orientations.

These microscale properties allowed for investigation into the macroscale failure mechanisms imparted by germination and three different predators: beetles, squirrels and orangutans. Details involving calculations presented below may be found in the original paper[3]. Germination is achieved via growth of an internal hydrostatic pressure which eventually drives a crack from the ends of the plug (crack initiation site) extending into the germination band (zone III). The pressure to drive this crack around the band is calculated by treating the shell as a thin-walled spherical pressure vessel and is estimated between 1.7-3.9 MPa. This

\footnotetext{
${ }^{1}$ Direction of loading.
} 


\section{seed interior}

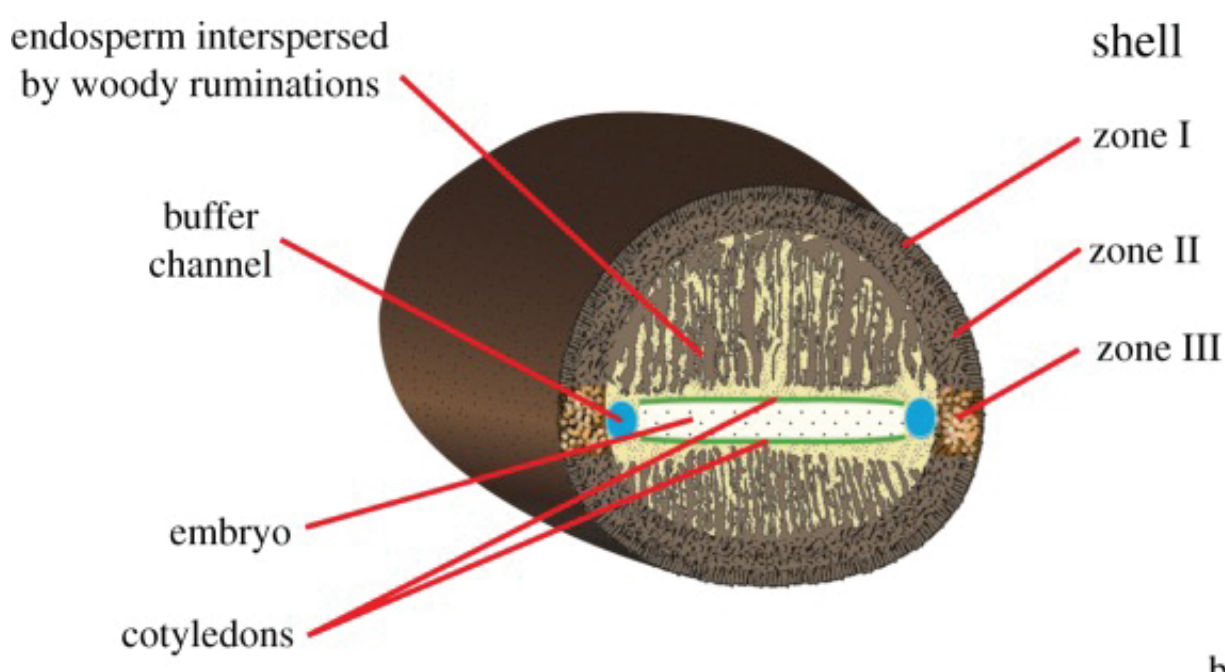

buffer channel stops crack in shell

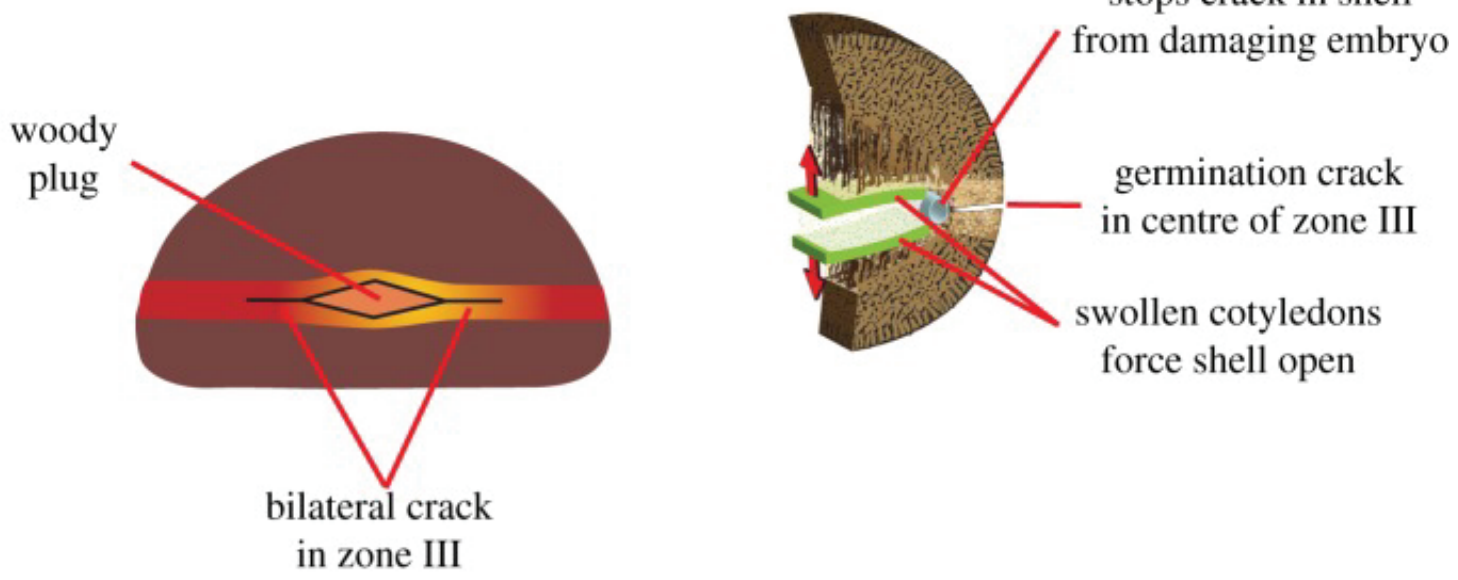

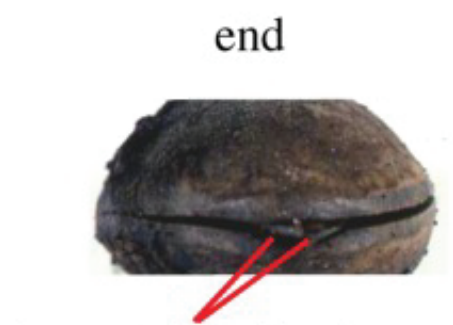

hypocotyl ejecting plug (split into two)

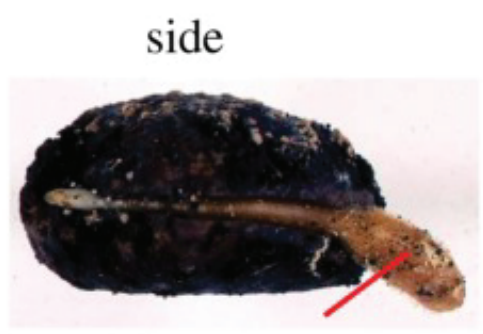

emerging hypocotyl

Figure 8.5: Architecture of M. parviflora. Areas where indentation is used to examine micro scale properties are zones I, II and III. Middle two nut diagrams show the woody plug and zone III band where the crack initiates during germination. The bottom two figure illustrate the germination process as the seed is ejecting the plug and hypocotyl[3]. 
(a)

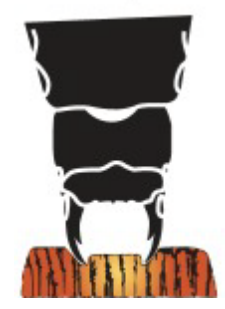

(b)

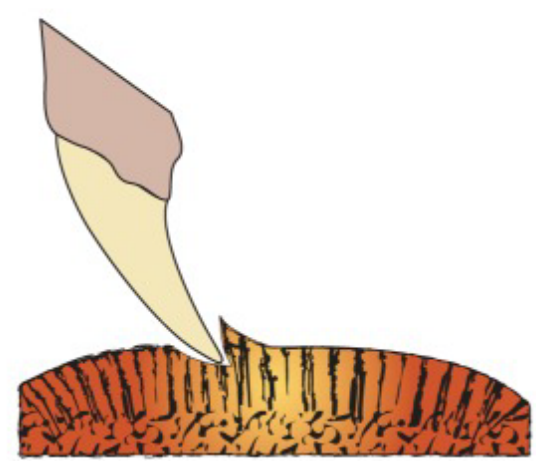

(c)

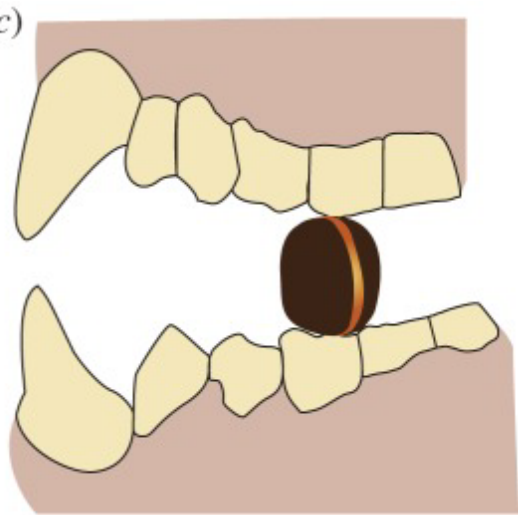

Figure 8.6: Illustration of predation mechanisms by beetles (a), squirrels (b) and orangutans(c). All of these predatory mechanisms happen over different length scales varying from $\sim 1 \mu \mathrm{m}$ to millimeters[3].

falls in the range of internal pressures measured in plant tissues (1-4 MPa) [80].

The shells of $M$. parviflora are attacked by specialized seed-boring beetles. Tunneling normal to the surface, they bore into the shell similar to the diagram in 8.6a. Once inside they lay eggs and die, as their mandibles are worn down during entry. Upon hatching, the larvae feed on the seed's endosperm, develop and mate[3]. The force exerted by the mandibles is estimated as $\mathrm{F} \sim 0.15 N$ by modeling the force to permanently damage a brittle surface via indentation. The maximum mandible force measured for similar beetles is in the range $F \sim 0.1-0.4 N[81]$. This suggests that shell properties lie at the minimum required to prevent insect boring except by the most specialized species.

Squirrels are known to be seed destroyers in other sites[82]. They gnaw on seeds which is similar to a chipping mechanism as demonstrated in $8.6 \mathrm{~b}$. Assuming a maximum bite force of $150 \mathrm{~N}$ and using an equation for chipping forces in brittle materials, the bite force is expected to produce chips on the order of $\sim 175-360 \mu \mathrm{m}$. This implies that the squirrels need to make at least 8 bites with close to maximum force just to penetrate the shell. It is expected that further effort is required to chip into the interior to access the embryo. Even though squirrels can consume the seed, the shell presents significant resistance, making it both an energy and time intensive source of food.

The feeding patterns of orangutans who eat $M$. parviflora have been extensively studied suggesting they spend between 13.3 and $18.2 \%$ of their total monthly feeding time consuming these seeds [83]. Additionally they are the hardest item which orangutans are known to consume [84]. Two different cracking methodologies have been observed while orangutans are eating these seeds. The first involves the seed being placed between the jaw with the germination band running from the bottom of the tooth to the top, similar to the graphic in figure 8.6. The force required to crack the nut in this configuration is estimated between 
$F \sim 1000-2000 N$ and makes use of the plug area as a pre-crack to initiate failure. The second method positions the seed in the opposite configuration where the band runs perpendicular to the biting plane. In this configuration the seed is assumed to fail via unstable growth of flaws on the inside of the nut, exacerbated by tensile forces imparted on them due to shell bending underneath the load points. The peak force at fracture is estimated as $F \sim 5000-6000$. Experiments using a UTM and cobalt-chrome tooth replicas have previously estimated the force to crack M. parviflora at 6000N (range 2700-8100 N)[85]. Despite orangutans' success in consuming these seeds, the high forces required show that significant resistance to biting is provided and can only be exploited, once again, by specialized predators.

These observations regarding $M$. parvifloras' ability to germinate while providing the maximum amount of protection against a wide array of predators points to evolutionary optimization. Weakness to any one of these predation mechanisms might cause a dramatic loss in the seeds' ability to repopulate which could result in extinction of the species. The use of nanoindentation has shed light on the role microscale properties and architectures play in the survival of this seed.

\subsection{Beam Dimensions and Results}

Table 8.2: 42nm beam dimensions

\begin{tabular}{cccc}
\hline \hline Beam ID & Length $(\mu \mathrm{m})$ & Width $(\mu \mathrm{m})$ & Thickness $(\mathrm{nm})$ \\
\hline 42a-1 & 196 & 20.73 & 42 \\
$42 \mathrm{a}-2$ & 196 & 20.73 & 42 \\
$42 \mathrm{a}-3$ & 196 & 20.73 & 42 \\
& & & \\
$42 \mathrm{~b}-1$ & 183 & 20.73 & 42 \\
$42 \mathrm{~b}-2$ & 178 & 20.73 & 42 \\
$42 \mathrm{~b}-3$ & 193 & 20.73 & 42 \\
& & & 42 \\
$42 \mathrm{c}-1$ & 197 & 20.73 & 42 \\
$42 \mathrm{c}-2$ & 196 & 20.73 & 42 \\
$42 \mathrm{c}-3$ & 196 & 20.73 & \\
\hline \hline
\end{tabular}


Table 8.3: $81 \mathrm{~nm}$ beam dimensions

\begin{tabular}{cccc}
\hline \hline Beam ID & Length $(\mu \mathrm{m})$ & Width $(\mu \mathrm{m})$ & Thickness $(\mathrm{nm})$ \\
\hline $81 \mathrm{a}-1$ & 193 & 20.97 & 81 \\
$81 \mathrm{a}-2$ & 193 & 20.97 & 81 \\
$81 \mathrm{a}-3$ & 192 & 20.97 & 81 \\
& & & \\
$81 \mathrm{~b}-1$ & 188 & 20.97 & 81 \\
$81 \mathrm{~b}-2$ & 188 & 20.97 & 81 \\
$81 \mathrm{~b}-3$ & 188 & 20.97 & 81 \\
& & & \\
$81 \mathrm{c}-1$ & 181 & 20.97 & 81 \\
$81 \mathrm{c}-2$ & 178 & 20.97 & 81 \\
$81 \mathrm{c}-3$ & 178 & 20.97 & \\
& & & \\
\hline \hline
\end{tabular}

Table 8.4: $232 \mathrm{~nm}$ beam dimensions

\begin{tabular}{cccc}
\hline \hline Beam ID & Length $(\mu \mathrm{m})$ & Width $(\mu \mathrm{m})$ & Thickness $(\mathrm{nm})$ \\
\hline 232a-1 & 188 & 20.68 & 232 \\
232a-2 & 187 & 20.68 & 232 \\
232a-3 & 190 & 20.68 & 232 \\
& & & \\
$232 \mathrm{~b}-1$ & 195 & 20.68 & 232 \\
$232 \mathrm{~b}-2$ & 194 & 20.68 & 232 \\
$232 \mathrm{~b}-3$ & 194 & 20.68 & 232 \\
& & & \\
$232 \mathrm{c}-1$ & 184 & 20.68 & 232 \\
$232 \mathrm{c}-2$ & 183 & 20.68 & 232 \\
$232 \mathrm{c}-3$ & 178 & 20.68 & 232 \\
& & & \\
\hline \hline
\end{tabular}


Table 8.5: $377 \mathrm{~nm}$ beam dimensions

\begin{tabular}{cccc}
\hline \hline Beam ID & Length $(\mu \mathrm{m})$ & Width $(\mu \mathrm{m})$ & Thickness $(\mathrm{nm})$ \\
\hline 377a-1 & 200 & 20.63 & 377 \\
$377 \mathrm{a}-2$ & 200 & 20.63 & 377 \\
$377 \mathrm{a}-3$ & 200 & 20.63 & 377 \\
& & & \\
$377 \mathrm{~b}-1$ & 184 & 20.63 & 377 \\
$377 \mathrm{~b}-2$ & 184 & 20.63 & 377 \\
$377 \mathrm{~b}-3$ & 180 & 20.63 & 377 \\
& & & \\
$377 \mathrm{c}-1$ & 168 & 20.63 & 377 \\
$377 \mathrm{c}-2$ & 171 & 20.63 & 377 \\
$377 \mathrm{c}-3$ & 171 & 20.63 & 377 \\
& & & \\
\hline \hline
\end{tabular}

Table 8.6: As-fabricated Elastic Modulus and Residual Strain

\begin{tabular}{cccc}
\hline \hline Beam ID & E $(\mathrm{GPa})$ & $\bar{\varepsilon}_{R}$ & $\varepsilon_{R}$ \\
\hline $42 \mathrm{a}-1$ & 153.3 & 241646 & 0.000925 \\
$42 \mathrm{a}-2$ & 147.5 & 313525 & 0.0012 \\
$42 \mathrm{a}-3$ & 152.8 & 274693 & 0.00105 \\
& $151.2 \pm 3.5$ & $276621 \pm 35978$ & $0.00106 \pm 0.00014$ \\
& & & \\
$81 \mathrm{a}-1$ & 179.5 & 83181 & 0.00122 \\
$81 \mathrm{a}-2$ & 191 & 98614 & 0.00144 \\
$81 \mathrm{a}-3$ & 181.3 & 103416 & 0.00153 \\
& $183.9 \pm 5.4$ & $95070 \pm 10573$ & $0.0014 \pm 0.00016$ \\
$232 \mathrm{a}-1$ & 186.3 & 12527 & 0.00159 \\
$232 \mathrm{a}-2$ & 183 & 12384 & 0.00159 \\
$232 \mathrm{a}-3$ & 178.1 & 10500 & 0.00131 \\
& $182.5 \pm 3.7$ & $11804 \pm 1131$ & $0.00149 \pm 0.00016$ \\
$377 \mathrm{a}-1$ & 178.6 & 6685 & 0.00198 \\
$377 \mathrm{a}-2$ & 178 & 5813 & 0.00172 \\
$377 \mathrm{a}-3$ & 186.8 & 6155 & 0.00182 \\
& $181.1 \pm 4.5$ & $6218 \pm 439$ & $0.00184 \pm 0.00013$ \\
& & & \\
\hline \hline
\end{tabular}


Table 8.7: 200C Annealed Elastic Modulus and Residual Strain

\begin{tabular}{cccc}
\hline \hline Beam ID & E $(\mathrm{GPa})$ & $\bar{\varepsilon}_{R}$ & $\varepsilon_{R}$ \\
\hline $42 \mathrm{~b}-1$ & 148.7 & 319087 & 0.0014 \\
$42 \mathrm{~b}-2$ & 153.8 & 323217 & 0.0015 \\
$42 \mathrm{~b}-3$ & 152.8 & 212265 & 0.00084 \\
& $151.7 \pm 2.9$ & $284856 \pm 62900$ & $0.00125 \pm 0.00036$ \\
$81 \mathrm{~b}-1$ & 193 & 87110 & 0.00135 \\
$81 \mathrm{~b}-2$ & 189.5 & 82661 & 0.00128 \\
$81 \mathrm{~b}-3$ & 185.2 & 85002 & 0.00131 \\
& $189.2 \pm 3.6$ & $84924 \pm 2226$ & $0.00131 \pm 0.00003$ \\
$232 \mathrm{~b}-1$ & 188.2 & 10988 & 0.0013 \\
$232 \mathrm{~b}-2$ & 192.2 & 11070 & 0.00132 \\
$232 \mathrm{~b}-3$ & 183.2 & 9855 & 0.0012 \\
& $187.9 \pm 4.5$ & $10638 \pm 679$ & $0.00126 \pm 0.00008$ \\
$377 \mathrm{~b}-1$ & 192.3 & 5591 & 0.00196 \\
$377 \mathrm{~b}-2$ & 185.3 & 3972 & 0.00139 \\
$377 \mathrm{~b}-3$ & 189.5 & 5320 & 0.00195 \\
& $189 \pm 3.5$ & $4961 \pm 867$ & $0.00176 \pm 0.00032$ \\
\hline \hline
\end{tabular}


Table 8.8: 300C Annealed Elastic Modulus and Residual Strain

\begin{tabular}{cccc}
\hline \hline Beam ID & $\mathrm{E}(\mathrm{GPa})$ & $\bar{\varepsilon}_{R}$ & $\varepsilon_{R}$ \\
\hline $42 \mathrm{c}-1$ & 198.6 & 636351 & 0.00241 \\
$42 \mathrm{c}-2$ & 196.7 & 661192 & 0.00253 \\
$42 \mathrm{c}-3$ & 205 & 697395 & 0.00267 \\
& $200.1 \pm 4.3$ & $664979 \pm 30698$ & $0.00254 \pm 0.00013$ \\
$81 \mathrm{c}-1$ & 197.6 & 175939 & 0.00294 \\
$81 \mathrm{c}-2$ & 197.5 & 162208 & 0.0028 \\
$81 \mathrm{c}-3$ & 200 & 144485 & 0.00249 \\
& $198.4 \pm 1.4$ & $160877 \pm 15769$ & $0.00274 \pm 0.00023$ \\
$232 \mathrm{c}-1$ & 197.5 & 19718 & 0.00261 \\
$232 \mathrm{c}-2$ & 200.3 & 19487 & 0.00261 \\
$232 \mathrm{c}-3$ & 197.7 & 18809 & 0.00266 \\
& $198.5 \pm 1.6$ & $19338 \pm 472$ & $0.00263 \pm 0.00003$ \\
$377 \mathrm{c}-1$ & 193.4 & 5702 & 0.00239 \\
$377 \mathrm{c}-2$ & 198.6 & 5778 & 0.00234 \\
$377 \mathrm{c}-3$ & 205.1 & 5895 & 0.00239 \\
& $199 \pm 5.9$ & $5792 \pm 97$ & $0.00237 \pm 0.00003$ \\
\hline \hline
\end{tabular}




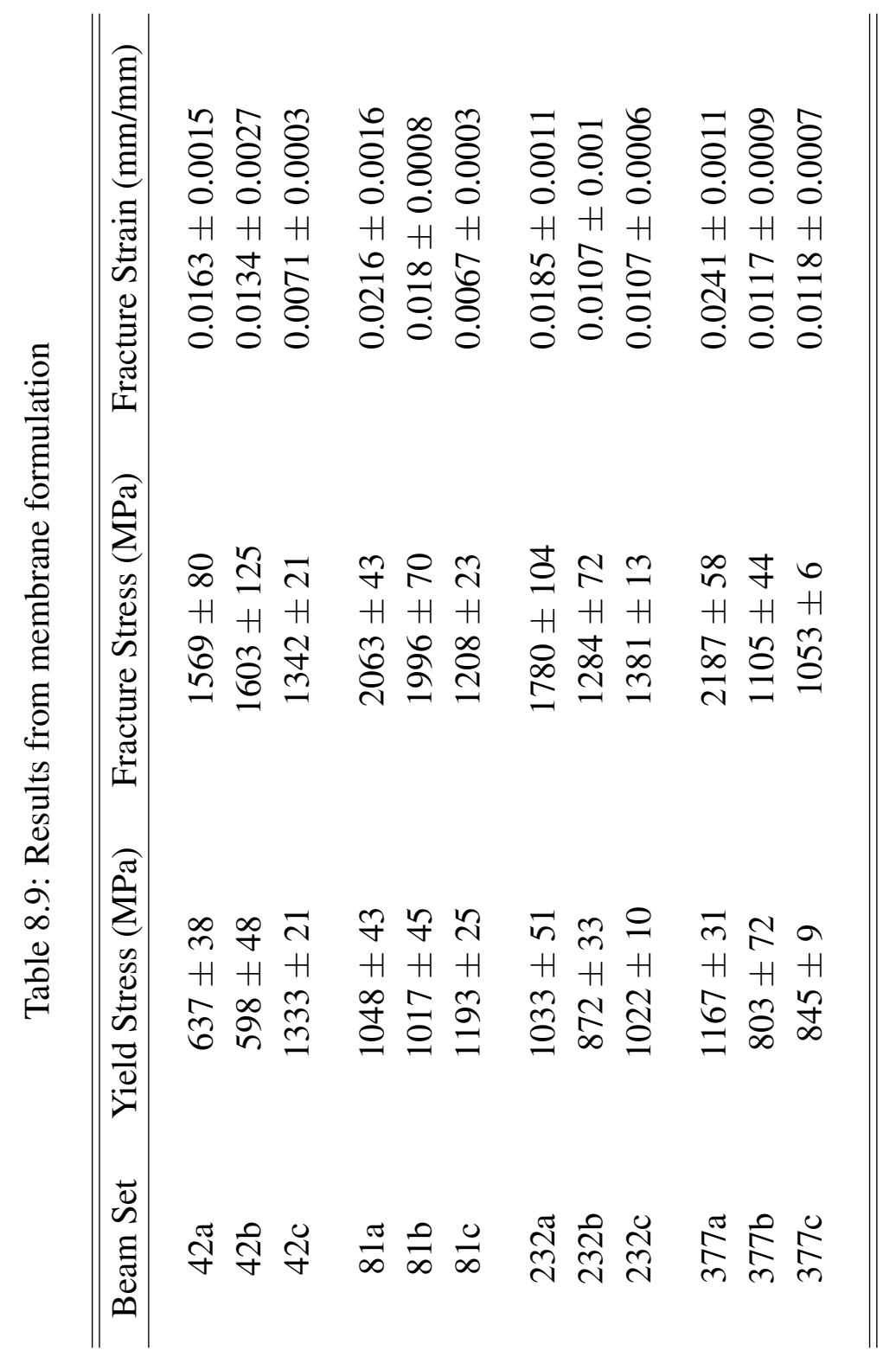

